\title{
Steer by ear: clinical and neurophysiological evaluation of a novel human-machine interface
}

\author{
Dissertation \\ for the award of the degree \\ "Doctor rerum naturalium" \\ of the Georg-August-Universität Göttingen
}

within the doctoral program Sensory and Motor Neuroscience of the Georg-August University School of Science (GAUSS)

submitted by

Leonie Schmalfuß

from Göttingen, Germany

Göttingen, 2017 


\section{Thesis Committee}

\section{Prof. David Liebetanz}

Supervisor, first referee

Department of Clinical Neurophysiology, University Medical Center, Göttingen

\section{Prof. Dario Farina}

Second referee

Department of Bioengineering, Imperial College, London

\section{Prof. Melanie Wilke}

Department of Cognitive Neurology, University Medical Center, Göttingen

\section{Members of the Examination Board}

\section{Prof. Hansjörg Scherberger}

Research Group Neurobiology, German Primate Center, Göttingen

\section{Dr. Arezoo Pooresmaeili}

AG Perception and Cognition, European Neurosciene Institute Göttingen (ENI)

\section{Dr. Igor Kagan}

Cognitive Neuroscience Laboratory, German Primate Center, Göttingen

Date of submission: December 2017

Date of oral examination: February 2018 


\section{Table of Contents}

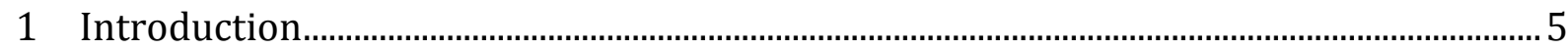

1.1 Traumatic spinal cord injuries.....................................................................................6

1.2 Control options of assistive devices for individuals with tetraplegia.........................9

1.3 Neuronal plasticity and functional recovery after spinal cord injuries ..................12

1.4 Neuronal changes with motor training in able-bodied and SCI subjects ................15

1.5 Transradial arm amputation and prosthesis control options ....................................17

1.6 The auricular muscles in the context of human-machine interfaces .......................23

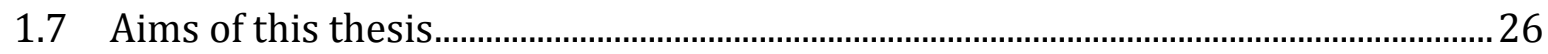

2 Steer by ear: proof of principle of the ACS for wheelchair control................................... 28

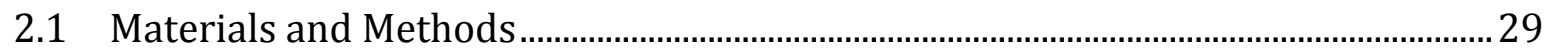

2.1.1 EMG recording and signal transmission in the ACS.............................................. 29

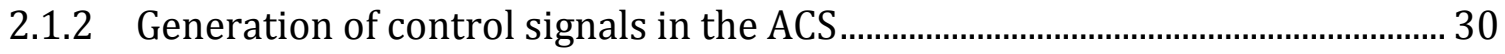

2.1.3 Generation of wheelchair driving commands ..................................................... 32

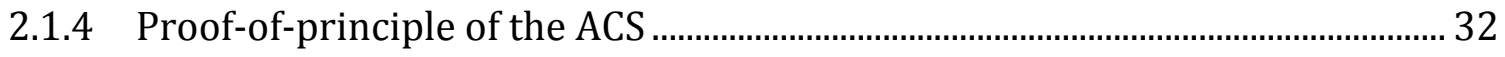

2.1.5 Training of voluntary control over the PAM ...................................................... 33

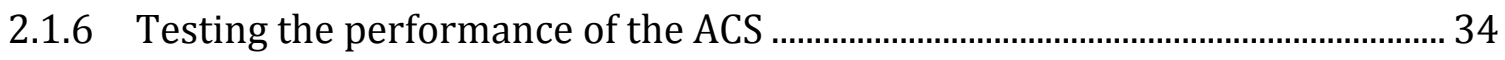

2.1.7 Powered wheelchair driving with the ACS .......................................................... 36

2.1.8 User evaluation of the ACS ..................................................................................... 37

2.1.9 Statistical Analysis............................................................................................. 38

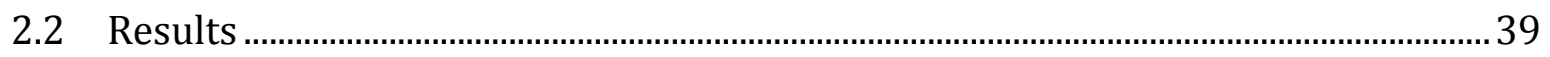

2.2.1 Training effects on ACS performance..................................................................... 39

2.2.2 Wheelchair driving with PAM EMG-generated control signals ......................... 45

2.2.3 Subject-reported outcomes on training and ACS performance .......................... 45

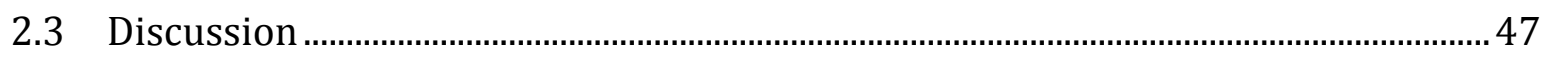

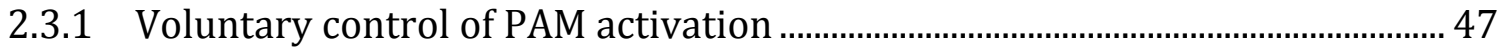

2.3.2 Application of the ACS for wheelchair control .................................................. 49

2.3.3 Evaluation of the ACS: social implications ……………………………………... 51

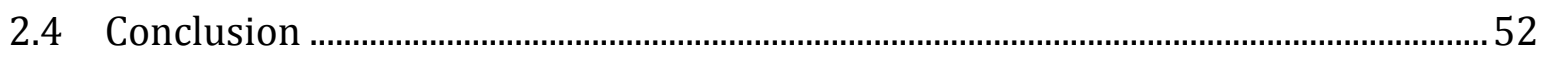

3 Independent control of two degrees of freedom in prosthetic hands with the ACS ... 53

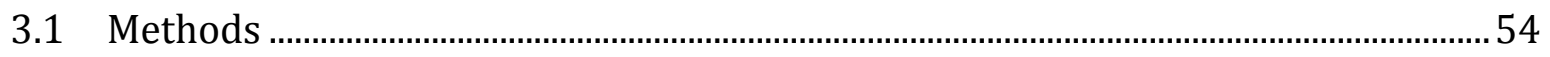

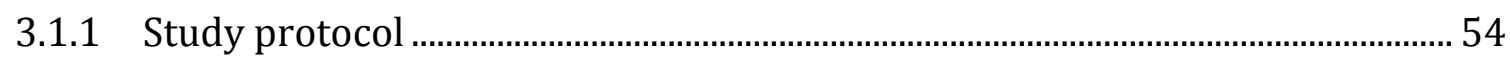

3.1.2 Prosthesis and control interfaces ……………………………………………... 55

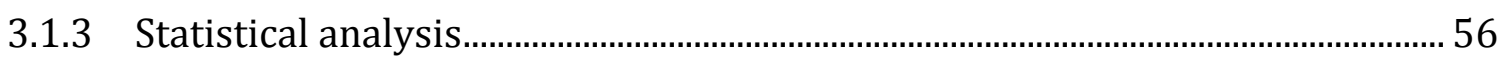

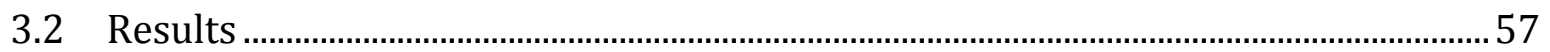

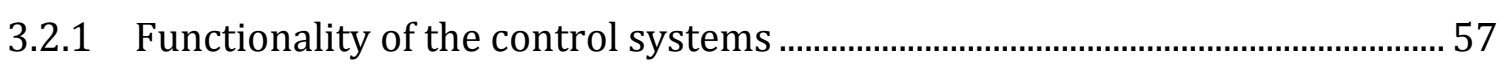

3.2.2 Influence of arm position: robustness with hACS................................................ 59

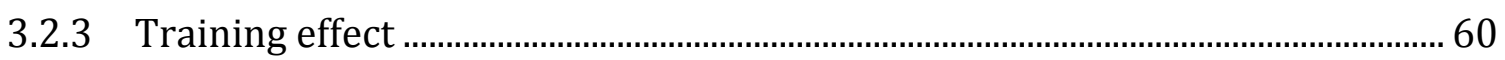

3.2.4 Results in a transradial amputee....................................................................... 61

3.3 Discussion and conclusions........................................................................................62

4 Neuronal effects of training the auricular control system for wheelchair control in subjects with tetraplegia. 


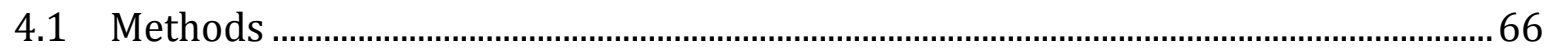

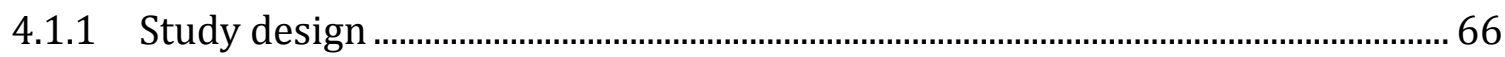

4.1.2 Software-based training and testing of control capacity of the auricular muscles

4.1.3 Training and testing of the ACS applied to wheelchair control ...................................... 68

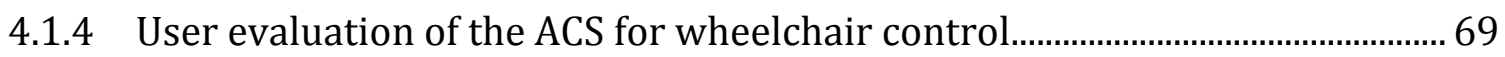

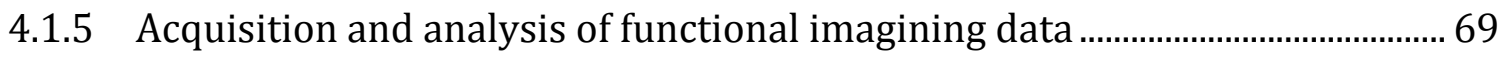

4.1.6 Robotic arm-controlled and image-guided TMS mapping procedure .............. 71

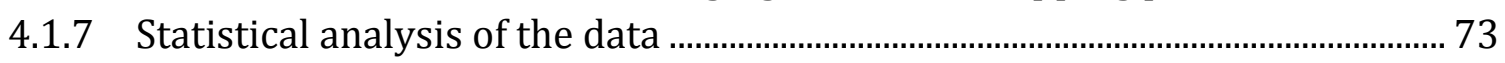

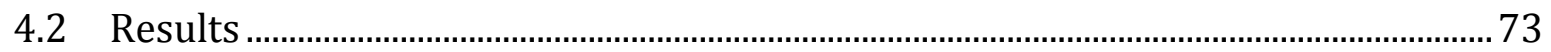

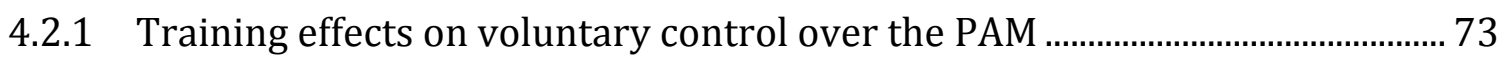

4.2.2 Training effects on wheelchair driving using the ACS ........................................... 73

4.2.3 User evaluation of the ACS for wheelchair control............................................... 74

4.2.4 Activation cluster size and centers of gravity during motor tasks in fMRI.... 75

4.2.5 Comparison of SCI-fMRI to data from able-bodied subjects ........................................ 81

4.2.6 Correlation of neuronal activation and behavioral performance ....................... 81

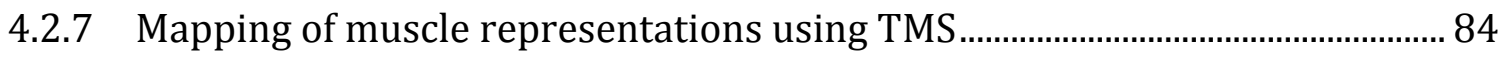

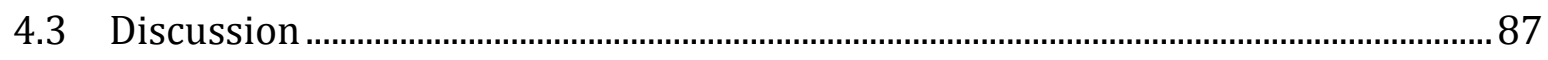

4.3.1 The ACS as wheelchair control: performance of SCI............................................. 87

4.3.2 Motor cortex reorganization in chronic SCI: superior PAM control as a

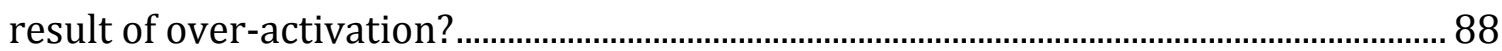

4.3.3 Training reduces motor cortex activity and increases performance ................90

4.3.4 Shifts of activation before and after training: which motor cortex areas

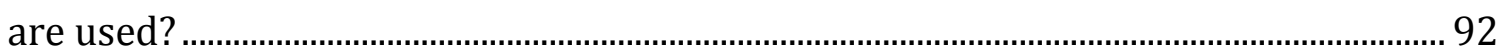

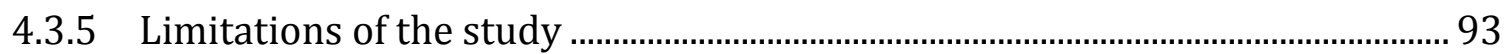

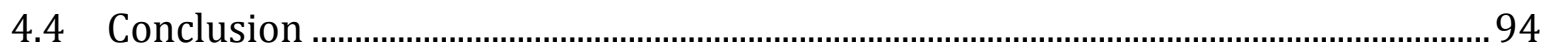

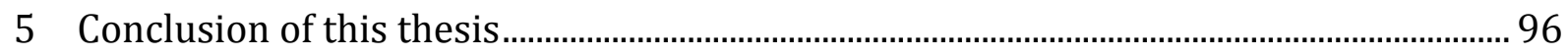

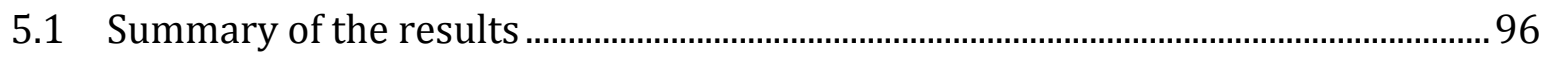

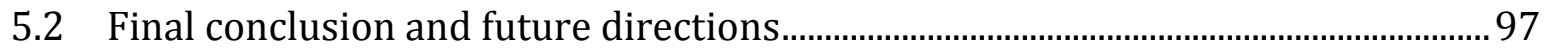

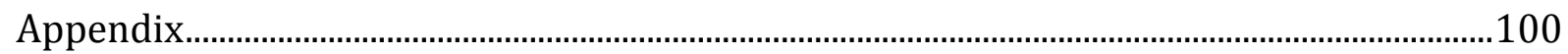

I) Questionnaire about ability to activate the PAM ............................................................ 100

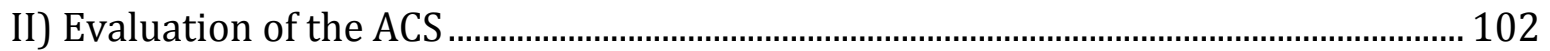

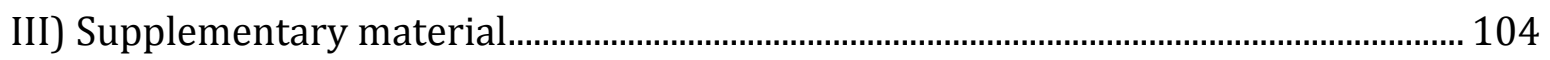

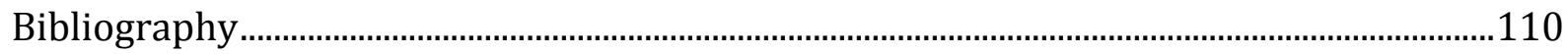

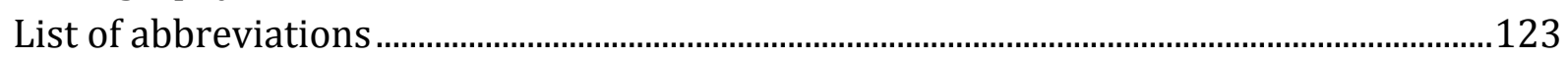

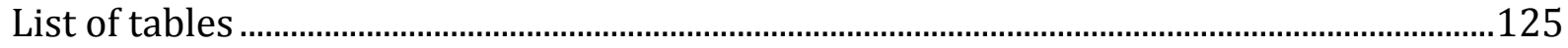

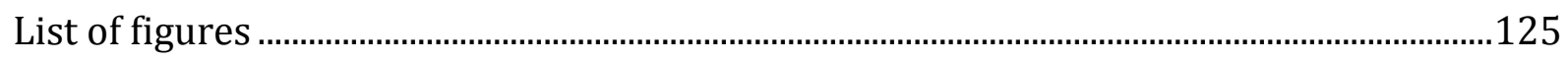

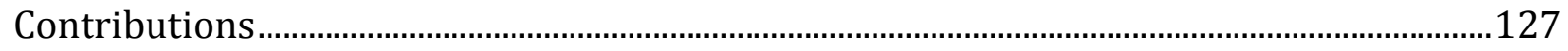

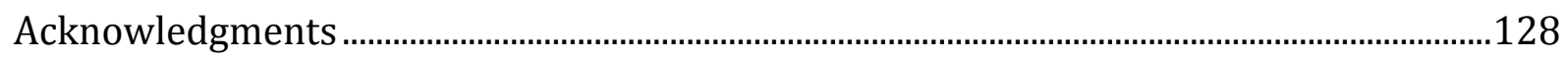

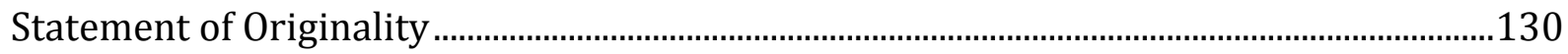

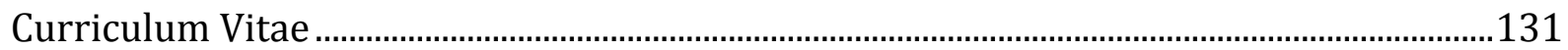




\section{Introduction}

Assistive devices are used to compensate for impaired bodily functions. These devices can help with communication, mobility or the use of electronic devices, such as mobile phones. Among the most used devices are wheelchairs and arm prostheses. Users of these devices depend heavily on them to lead a normal life. However, when it comes to controlling assistive devices, the available options do not always satisfy. For example, individuals with high-level tetraplegia can find wheelchair control challenging because the available control options depend on functions that might be impaired or interfere heavily with other activities. Clinically established hand prosthesis controls on the other hand can only provide control over limited movements. These shortcomings in control options cause limited use or even rejection of both wheelchairs and arm prostheses, impairing participation and life quality.

This thesis presents an alternative control option for assistive devices, which uses the muscle signals from the human auricular muscles. The auricular control system (ACS) can provide independent, proportional and simultaneous control over two degrees of freedom (DOF), which can be used for different assistive devices. Little is known about the nature of the auricular muscles; but due to their isolated position and vestigial nature, interference with other activities is minimal. This thesis addresses the feasibility of the ACS in two relevant clinical indication fields, namely wheelchair and prosthesis control, and whether the ACS can solve some of the presented problems with control of assistive devices.

In the following introduction the basic concepts necessary for understanding the presented studies are stated. The first study (chapter 2) addresses the characteristics of the auricular muscles: whether precise control over these vestigial muscles could be learned and trained and if these muscles can generate suitable control signals for wheelchair control. The second study (chapter 3) presents results from an application of the ACS to prosthesis control. In the third study (chapter 4) the ACS is tested in a clinical cohort of individuals with tetraplegia. In these subjects who present unique brain reorganization due to the loss of limb functions, neuronal correlates of the motor training are investigated with functional magnetic resonance imaging (fMRI) and transcranial magnetic stimulation (TMS). Finally, chapter 5 summarizes and discusses the overall findings and offers a future direction of further research. 


\subsection{Traumatic spinal cord injuries}

Each year between 10.4 to 83 new cases of spinal cord injuries (SCI) per million inhabitants occur (Wyndaele \& Wyndaele, 2006). The percentage of causes differs between studies, but most often the trauma is due to vehicle related accidents (38-55\%), falls (23-30,5\%), acts of violence (primarily gunshot wounds, 13-13,5\%), sports (9\%), surgical origins (5\%) and other (4\%) (Burke, Linden, Zhang, Maiste, \& Shields, 2001; National SCI Statistical Center, 2015). Young men have a specially high risk of spinal cord injury, with the ratio of males to females being $3: 1$ to $4: 1$ and the average age at injury between 33 (Wyndaele \& Wyndaele, 2006) to 42 years of age (National SCI Statistical Center, 2015).

When the spinal cord is severed, efferent motor signals from the brain can no longer reach the output muscles (Figure 1). Injuries can occur at any level of the spine and can be classified as a complete injury with total loss of motor and sensory function; and incomplete with some signals from the brain still able to bypass the injured spinal cord. As the height of lesion increases, there is a corresponding increase in the number of body parts that are affected. From each vertebra a spinal nerve emerges which carries motor commands from the brain to the muscles (efferences) and sensory information from the body parts to the brain (afferences). The level of injury sustained by a person is defined as the last level of full sensory and motor functions. Below that vertebra, the efferences and afferences of the following spinal cords are blocked. Injuries at lumbosacral level lead to weakness of the legs and dysfunction of several bodily functions. Lesions of the thoracic vertebrae cause paralysis of the legs. Most severely, when the cervical vertebrae are affected, individuals lose control over their arms and possibly over autonomic lung function. This extensive loss of motor and sensory control over all four limbs happens in 30-60\% of all spinal cord injuries, with a tendency to increase in recent years (Burke et al., 2001; National SCI Statistical Center, 2015; Wyndaele \& Wyndaele, 2006). Individuals that suffer from an injury at the C8 level have difficulties controlling the hand functions and, in case of a complete injury, have no control over the arm extensor and below. Injuries on C7 level still allow the thumbs to function, but the rest of the hand is paralyzed. This important function is lost with C6 injuries, as well as the ability to flex the wrist. Complete paralysis of the arm happens with lesion of C5. At the C4 level, impairment of other autonomous functions such as 
bladder and bowel control and diaphragm function add up to the motoric impairment of the shoulders. At the C3 level, individuals depend on a ventilator for breathing. At the C2 and C1 level, patients may even lose the ability to speak and all control over the head and neck position.

Besides the resulting immobility, spinal cord injuries have a major impact over the health of the affected individuals. Complications include respiratory and cardiovascular problems, muscle spasms and muscle atrophy, bladder infections and pressure sores. Life expectancy is significantly reduced due to these complications, with pneumonia and septicemia as the leading causes for premature death (National SCI Statistical Center 2015). Involuntarily, individuals with SCI place an enormous burden on health care systems. They require continuous physiotherapy to prevent muscle atrophy and contractures, treatment of secondary complications, and in most cases personal assistance around the clock. The lifetime cost of a person with SCI for the health care system is estimated between 1.5 and 4.8 million dollars, depending on the severity of the handicap and age at injury (National SCI Statistical Center, 2015). 

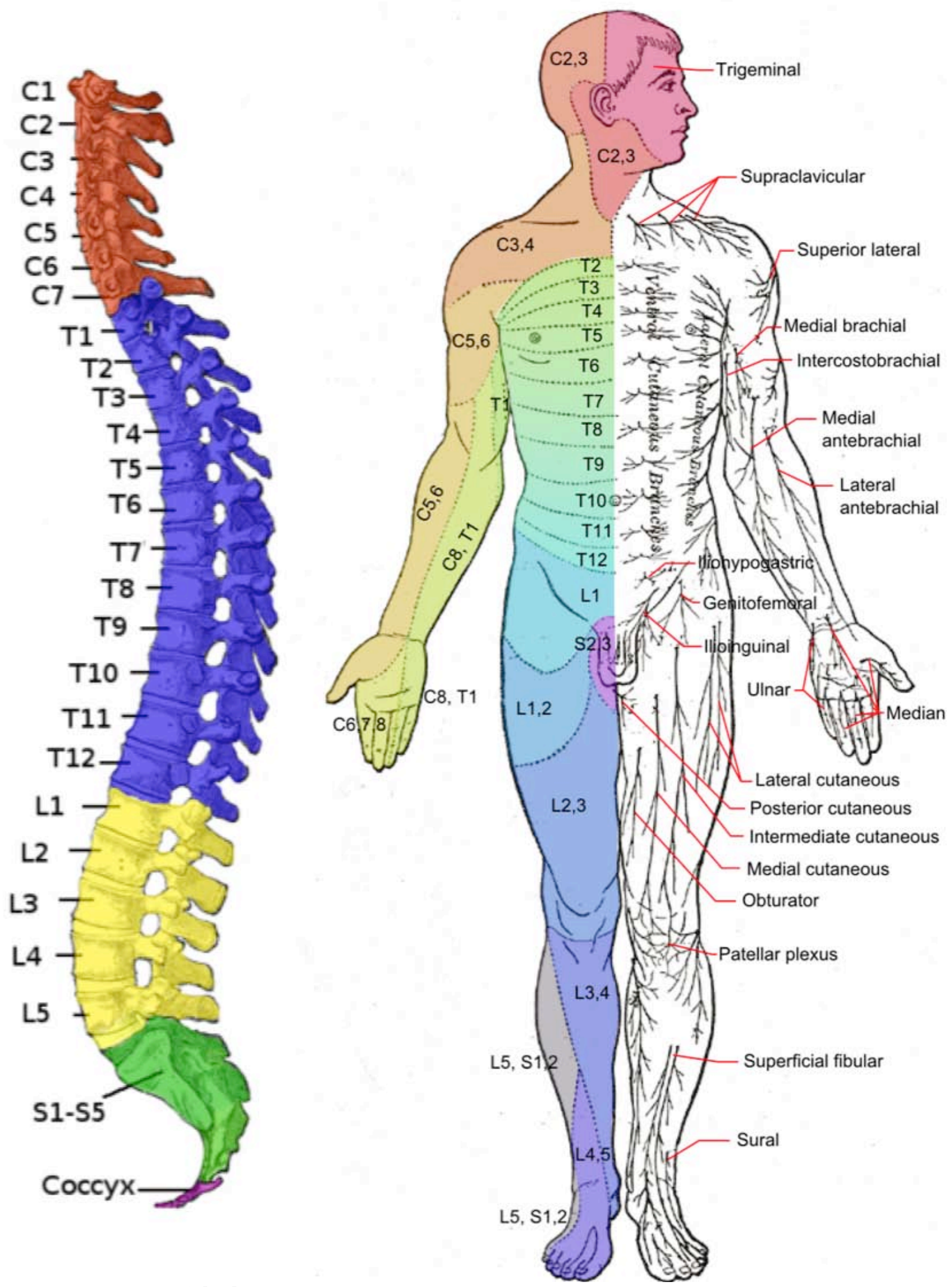

Figure 1) Anatomy of the human spine.

The vertebrae are named after their position ( $\mathrm{S}=$ Sacral, $\mathrm{L}=$ lumbar, $\mathrm{T}$ =thoracic, $\mathrm{C}=$ cervical) and numbered (left). The spinal nerves innervate dermatomes (sensory areas, left on human) and myotomes (muscle groups, right). When a vertebra is injured both sensory input to the brain and motor output from the brain of the corresponding areas are impaired. (Pictures from https://commons.wikimedia.org/wiki/File:Gray_111_-_Vertebral_column-coloured_labels.png. and https://commons.wikimedia.org/wiki/File:Dermatomes_and_cutaneous_nerves_-_anterior.png Retrieved 08.12.2017.) 


\subsection{Control options of assistive devices for individuals with tetraplegia}

Mobility is an important factor for individual wellbeing. Not surprisingly, it has been found that individuals with tetraplegia suffer from a lower quality of life and have more depressive episodes (Craig, Tran, \& Middleton, 2009). Concurrently, the self efficacy and sense of control are significantly reduced (Middleton, Tran, \& Craig, 2007), which are important factors for mental wellbeing. The use of assistive devices has a positive influence on the quality of life, by providing greater independence (Scherer \& Cushman, 2001). For individuals with tetraplegia, the electric wheelchair is the most important assistive device, as it provides basic mobility and allows affected individuals to return to some kind of normality, for example living at home. Although wheelchairs are thus indispensable, in daily use they are often seen as an obstacle and can even limit participation (Chaves et al., 2004). Complaints from SCI wheelchair users include the size of the wheelchair, which impedes access to shops and houses, low maximum speed and electronic problems (Post, van Asbeck, van Dijk, \& Schrijvers, 1997). Even experienced wheelchair users have problems e.g. with static or dynamic obstacles (Torkia et al., 2015). In a survey with 200 practicing clinicians it was reported that after completing wheelchair training, $40 \%$ of the regular electric wheelchair users find steering tasks, such as navigation through doors or into an elevator, difficult (Fehr, Langbein, \& Skaar, 2000). 10\% of the patients could not complete tasks of daily living with the wheelchair at all. The authors conclude that available control options are apparently insufficient. Additionally, they identified a group of patients for whom no control option whatsoever exists. These patients lack the motor skill, or strength, to make use of the available control systems. The most used control interfaces are joysticks for those less impaired, and sip-and-puff or chin-control (Figure 2) in tetraplegia (Fehr et al., 2000). In sip-and-puff, steering signals are produced by inhaling or exhaling into a small tube, but the autonomic control over the diaphragm can be impaired from C5 lesions and higher (Arshak, Buckley, \& Kaneswaran, 2006). For chin or head control, sufficient control over the neck and shoulders is needed, which can be compromised by C4 lesions and higher. 


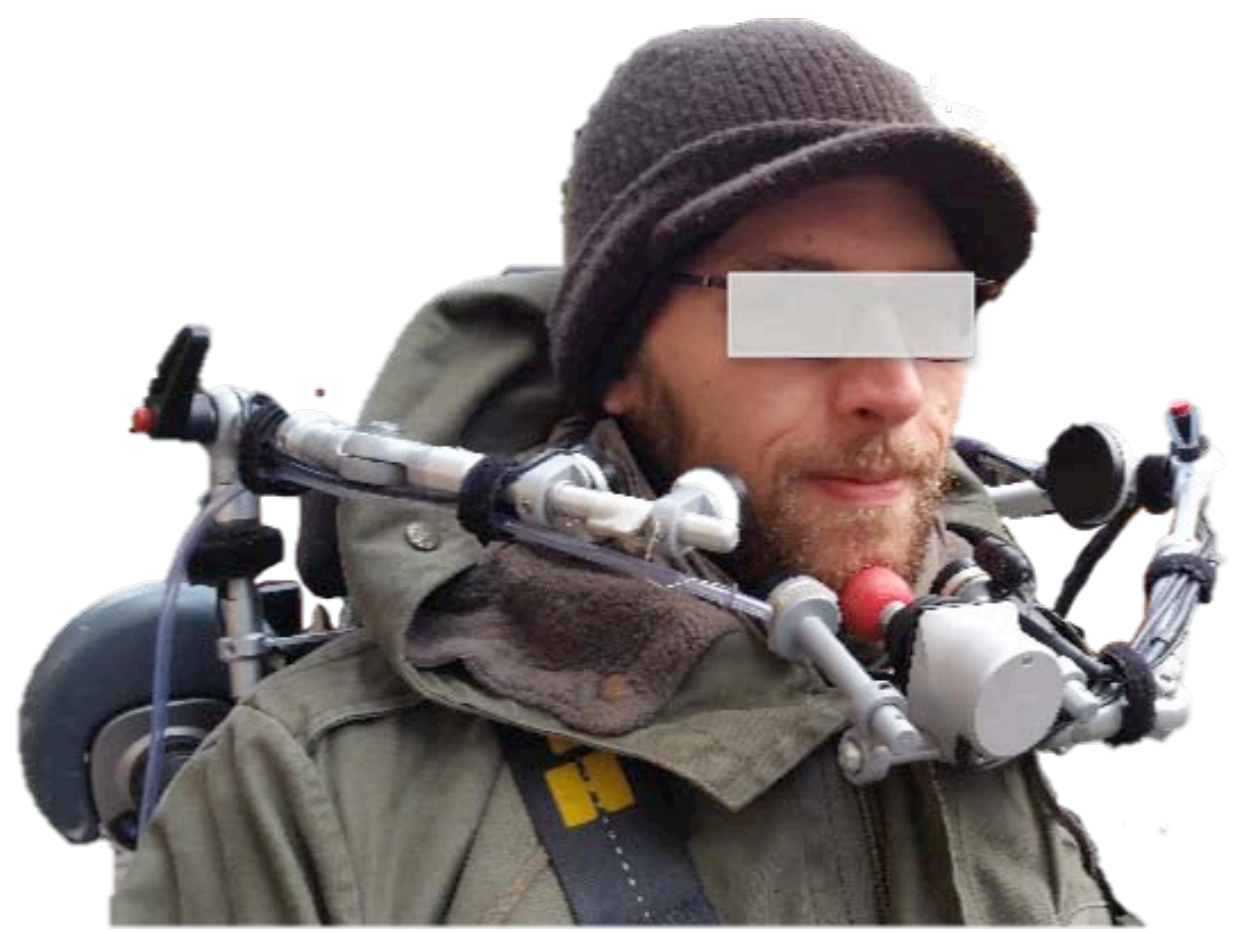

Figure 2) Individual with tetraplegia using a chin control.

The person can control the wheelchair by pushing a softball joystick with the chin. Additional buttons in front and to the left and the right of the cheeks serve e.g. for pulling the control set up to the side when the individuals wants to eat. This control is one of the most used in high-level tetraplegia, because it enables in a continuous, proportional control over the wheelchair. However, in many cases, control over the neck muscles is insufficient for generating precise signals.

With the advent of more complex electronic options, considerable effort has been put into the development of new systems and alternative control strategies. The most appealing approach is perhaps the Brain-Computer-Interface (BCI), where evoked or spontaneous brain waves can be used to control word-processors (Birbaumer et al., 1999; Wolpaw, Birbaumer, McFarland, Pfurtscheller, \& Vaughan, 2002), prostheses (Velliste et al., 2008) or even wheelchairs (Huang et al., 2012; J. D. R. Millán et al., 2009; Tanaka, Matsunaga, \& Wang, 2005). However, a limitation to this method is the complex setting required to record evoked EEG signals and the resulting low information transfer rate (J. D. Millán et al., 2010; Wolpaw et al., 2002), which are considered too slow for complex tasks like driving a wheelchair in a real-life setting (J. D. Millán et al., 2010). Asynchronous control uses a different approach by recording self-paced signals that do not depend on external cues. Here, specific brain activities for certain mental tasks (e.g. hand movement, relaxation) are used as specific steering commands (Ortiz-Rosario \& Adeli, 2013). This approach ensures more flexibility, since it does not depend on a 
specific environment, but reliable signal generation is still an issue. Another drawback of BCI that is still applicable to the asynchronous control, is the extremely long training time (Donoghue, 2008). Even after several months of training, reliability remains a problem that also affects security of BCI-controlled assistive devices (Diez et al., 2013).

Intelligent wheelchairs ("shared control") are used to moderate these problems. Shared control can help in complex situations, such as when the wheelchair identifies an obstacle and then automatically avoids it. Although this approach might increase safety, it inevitably reduces the users control over the wheelchair. However, the results certainly depend on the intelligence of the implanted system. With current research efforts, the performance could in future provide an intuitive, supporting system that does not intervene too dominantly. In the context of BCI, it does not solve the problems of difficult signal generation. First reports of combined BCI-shared control demonstrate that even simple tasks are challenging. Even after 20 calibration sessions, approaching a target with a two meter distance was only possible in $27-53 \%$ of trials, and an approach of one meter only in $7-37 \%$ of runs (J. D. R. Millán et al., 2009). Thus the clinical applicability and particularly the suitability for everyday use remain uncertain (Abbott, 2006; Ryu \& Shenoy, 2009). Movement artifacts and an unstable recording environment make BCI difficult for wheelchair use. This technology might hence be more adequate for the control of other assistive devices like computers and robotic arms (Donoghue, 2008).

Alternative innovative systems have the advantage of easier implementation and less training costs. Two research groups have proposed a tongue control, where the tongue position is decoded with a mouthpiece and several pre-set activities can be activated by relocating the tongue (Lontis et al., 2010; Struijk et al., 2009; Yousefi, Huo, \& Ghovanloo, 2011; Yousefi, Huo, Kim, Veledar, \& Ghovanloo, 2012). Although this system would even allow subjects with high tetraplegia control over assistive devices, it has two disadvantages. Firstly, signal generation is not flexible, only pre-set activities can be actuated. In the case of wheelchair driving, this means e.g. a fixed $90^{\circ}$ turn without the possibility for readjustment. Secondly, it interferes heavily with daily activities and most importantly, social interaction. The same applies to another proposed control option, that is activated by sniffing (Grafton, 2010; Plotkin et al., 2010). In some severe cases, such as locked-in-syndrome, these options might improve the patients' situation, but 
they are not a viable solution to the control problem experienced by most individuals with high-level tetraplegia.

\subsection{Neuronal plasticity and functional recovery after spinal cord injuries}

Traumatic spinal cord injuries lead to a loss of sensory and motor information from those body parts that are connected to the brain with spinal nerves below lesion level (Nardone et al., 2013). In addition, motor commands from the brain to these body parts are blocked on the spinal level (Figure 3). These changes in afferent and efferent connections lead to major brain reorganization, especially in the sensory-motor system (Kokotilo, Eng, \& Curt, 2009). In healthy humans, it has been shown that the somatotopical organization of the primary motor cortex (i.e. each body part has a specified area) is influenced by frequency and proficiency of use: when a body part is used more and with more mastery, the responsible area expands (Elbert, Pantev, Wienbruch, Rockstroh, \& Taub, 1995), invading the neighboring body representations. The organization of the primary motor cortex (M1) is hence not static. In the post-SCI brain, neuronal plasticity takes place as the brain adapts to the changed flow of information from the central nervous system. Shifts of activation have been reported by a majority of studies on brain reorganization after SCI (Kokotilo et al., 2009). However, the direction of the reported shift was either in the direction of the deafferent limb or in a posterior direction (Figure 3). Those studies reporting a posterior shift argued that the primary sensory cortex (S1) might compensate the axon loss of the M1 due to the trauma and hence cause a posterior shift (Kokotilo et al., 2009). Green et al. argue that the pyramidal axons descending from the S1 might be spared from the spinal trauma due to a more posterior location. They report a case where the posterior shift in a paraplegic was reversed after recovery (Green, Sora, Bialy, Ricamato, \& Thatcher, 1998). It thus seems that with a higher, more severe injury, the brain has to access extra help from the sensory motor cortex for motor command. This shift into the S1 was later confirmed with fMRI for paraplegic subjects (Cramer, Lastra, Lacourse, \& Cohen, 2005; Turner, Lee, Schandler, \& Cohen, 2003). Interestingly, sensory representations on the S1 did not shift, indicating that these areas are capable of multitasking. 


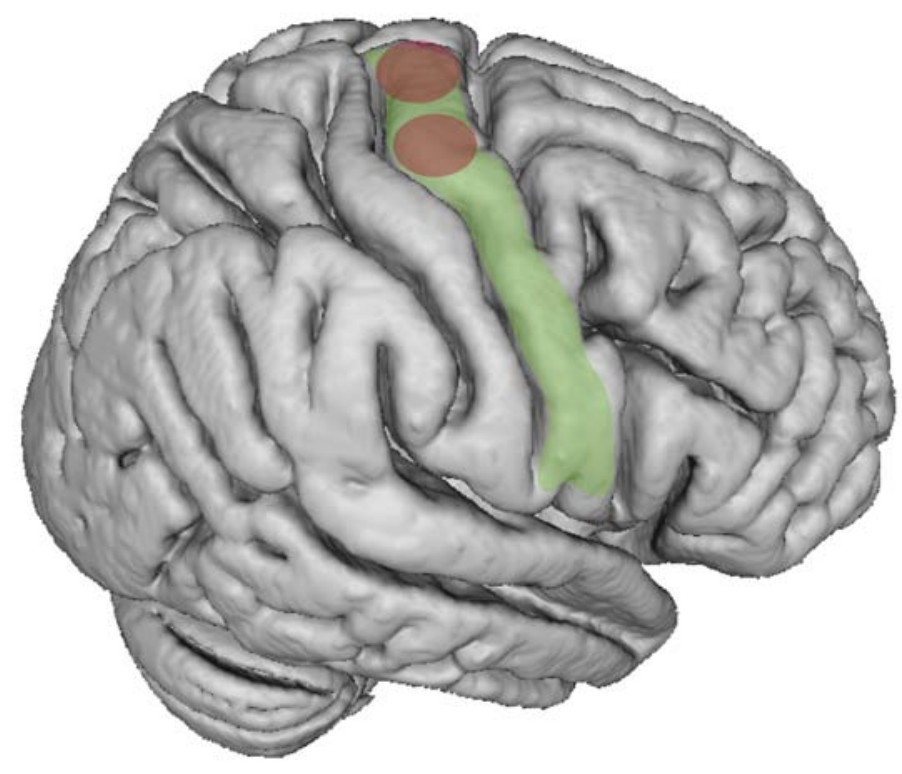

Figure 3) The motor cortex (green) on a human brain with areas typically deefferented in individuals with tetraplegia (red).

View on the right hemisphere of a standard MNI brain. In individuals with tetraplegia, typically the hand area (lower red spot) and the trunk area (higher red spot) as well as the leg and foot area which expands into the medial part of the brain (not in view) are deefferented, i.e. the signal output to the corresponding body parts is blocked. The primary sensory cortex, which in some cases compensates for motor cortex function loss, is located behind the motor cortex.

The contrasting shift into the deefferent limb region could possibly be a use of additional neuronal resources. Apparently, when parts of the motor cortex are unoccupied for some time, neighboring regions tend to "invade" the unoccupied area (e.g. the hand area shifts into the deefferent leg area (Bruehlmeier, 1998; M Lotze, Laubis-Herrmann, \& Topka, 2006; Mikulis et al., 2002)). Kokotilo et al. also suggest that the direction of the shift may depend on lesion level, e.g. lower lesions cause a shift into the deefferent area and higher lesions lead to additional recruitment of the S1. However it is still not clear, since there have been shifts into the deefferent leg area in subjects with tetraplegia (Freund, Weiskopf, et al., 2011) and posterior shifts in lower lesion subjects (Cramer et al., 2005; Turner et al., 2003).

Besides the shift in activation, the amount of motor cortex activation regarding activation size and strength seems to be influenced by the post-SCI brain reorganization. Several studies demonstrate a greater cluster size of motor task related activation of the M1 in SCI subjects when compared to able-bodied subjects. For example, Lundell et al. found increased M1 cluster activation when subjects with incomplete SCI executed residual movement of their ankle (Lundell, Christensen, et al., 2011). Comparably, 
greater M1 activations in SCI than in healthy subjects were found during hand activation (Freund, Weiskopf, et al., 2011). However there are some studies that report similar motor activation as with able-bodied subjects. For lip movement, Mattia et al. did not find differences in activation sizes between able-bodied participants and those with tetraplegia (Mattia et al., 2006). Likewise, Sabbah et al. recorded no change in quadriplegic subjects when they attempted to move their feet (Sabbah et al., 2002). In a similar task, there was even reduced M1 activity in subjects with higher lesion levels (Hotz-Boendermaker et al., 2008). Turner et al. found stronger M1 activation, but no cluster size increase during hand movement (Turner et al., 2003). Jurkiewicz et al. also found an irregular activation pattern with reduced M1 activity and increased secondary motor areas (Jurkiewicz, Mikulis, McIlroy, Fehlings, \& Verrier, 2007). The over activation of secondary motor areas such as S1, cerebellum, pre-motor cortex (PMA), supplementary motor cortex (SMA) and Thalamus has been confirmed by others (HotzBoendermaker et al., 2008; Kokotilo et al., 2009). A reason for the heterogeneity of the results lies in the diversity of the subjects. The clinical characteristics of the included SCI subjects vary widely with regard to lesion level, time to injury, completeness of injury and thus remaining motor functions, and type of spinal cord injury (Kokotilo et al., 2009). It has been suggested that motor cortex activity increases with increasing lesion level (Bruehlmeier, 1998; Ghosh et al., 2009; Lundell, Christensen, et al., 2011). Conversely, the extent of the spinal cord injury correlates with greater motor cortex activation (Freund, Curt, Friston, \& Thompson, 2013; Lundell, Christensen, et al., 2011). Another source of variability is the employed motor task, which ranges from attempted movement of muscles below lesion level, motor imagery or executed movement of muscles above lesion. Thus reduced M1 activation might be found in attempted foot movement in tetraplegic (Hotz-Boendermaker et al., 2008), but not in quadriplegic individuals (Sabbah et al., 2002). Although a final conclusion is difficult given these variations, a systematical review of 25 neurological studies on SCI subjects identified that there is evidence for an increase in motor cortex activity and secondary motor areas in SCI subjects, but that the exact dependency on factors such as lesion level and time to injury are not fully understood (Kokotilo et al., 2009).

The same applies to the functionality of these brain reorganizations. The normal functioning of neuronal plasticity suggests that when some areas do not receive any input or can not execute their motor commands, these areas typically diminish and are 
overtaken by the still active functions of the motor cortex (Kokotilo et al., 2009). The over-activation of the primary motor cortex has been interpreted as a functional compensation for the lesion of neighboring corticospinal fibers (Lundell, Barthelemy, et al., 2011). It has also been interpreted as more of an effort for the motoric system to still produce motor output (Moxon, Oliviero, Aguilar, \& Foffani, 2014) or simply the result of an overuse of the still active muscles (Sabre et al., 2013).

On the other hand these brain adaptions can also lead to negative effects. It has been shown that a transient deafferentiation of extremities can cause enhanced excitability, which has been interpreted as reduced inhibition from interneurons (Ziemann, Corwell, \& Cohen, 1998). In addition, it was found that the GABAergic intracortical inhibitory circuit is reduced in the motor cortex in subjects with SCI. This circuit is important for normal motor output, for example in movement preparation or movement suppression (Roy, Zewdie, \& Gorassini, 2011). It has been suggested that these "abnormal features" of brain activation might represent a loss of motor cortex function (Silva, Sousa, Reis, \& Salgado, 2014), which further disables the motor output in SCI subjects (e.g. speed of movement, strength patterns (Nardone et al., 2015; Wrigley et al., 2009)).

The resulting over-activation of motor cortex might also be a hindrance in functional recovery (Cramer et al., 2005; Hotz-Boendermaker et al., 2008; Nardone et al., 2015) and influence other factors such as for neuropathic pain and spasticity (Sabre et al., 2013; Silva et al., 2014). In addition, deafferentiation can lead to neuron loss in the affected areas (Freund, Weiskopf, et al., 2011). These permanent anatomical changes might additionally limit functional recovery (Wrigley et al., 2009).

\subsection{Neuronal changes with motor training in able-bodied and SCI subjects}

Although findings are heterogeneous, research on brain plasticity induced by spinal cord injuries is abundant. The effects of motor activity on the reorganized post-SCI brain are however less well investigated. This is particularly surprising, since physiotherapy plays a crucial part in rehabilitation and has been shown to improve motor outcome and residual functions even in severely impaired individuals. Given that the post-SCI brain functions so differently from the healthy brain, neuronal correlations of motor training 
might have an impact on the physiotherapeutic intervention. Similarly, findings from neuroplasticity studies on the spinal cord have had a significant influence on rehabilitation practice. Since medical reversion of the spinal cord injury is not possible (Nardone et al., 2015), physiotherapy has long focused mostly on compensatory skills (Behrman, Bowden, \& Nair, 2006), i.e. to bypass incapacitated actions with those that are still possible. The finding that motor activity of the deafferent limbs, e.g. by passively moving them, could induce spinal cord plasticity (Edgerton, Tillakaratne, Bigbee, de Leon, \& Roy, 2004) has led to a paradigm shift to rebuilding instead of compensatory strategies (Behrman et al., 2006) and the emergence of new therapies, e.g. locomotor training. Here, paralyzed individuals are put to walk with adequate supportive equipment, and efferent sensory information from below the lesion level induces not only spinal cord changes, but also improve other non-related motor skills and spasticity, even in severely paralyzed subjects (Lim \& Tow, 2007; Moxon et al., 2014).

A better understanding of training-related brain reorganization in SCI subjects is hence mandatory and missing (Dunlop, 2008; Moxon et al., 2014; Silva et al., 2014). Thus, in order to develop cost-effective, improved rehabilitation therapies whilst possibly improving secondary complications (Dunlop, 2008), more information has to be collected and evaluated. It has been shown that in the post-SCI brain, additional brainderived neurotropic factor (BDNF) is available, which enhances neuronal plasticity (Dunlop, 2008). This readiness of the brain could be advantageous when captured by training.

Motor training studies with healthy subjects have shown different involvement of the primary motor cortex in the process of skill acquisition. Apparently in the first stages, called "fast learning", the M1 activity decreases in what is interpreted as a habituation effect (Dayan \& Cohen, 2011; Karni et al., 1995; Ungerleider, 2002). When the task is automated, M1 activity increases. Long-term effects have been reported conflictingly: some studies reveal an increase, some a decrease in M1 activity (Lohse, Wadden, Boyd, \& Hodges, 2014). The involvement of the M1 seems to be heavily task-dependent: serial reaction time tasks lead to increased M1 activation even after training is finished, while sensorimotor tasks result in a smaller increase (Hardwick, Rottschy, Miall, \& Eickhoff, 2013). Other groups state that an enhancement of M1 depends on learning qualities, and that a long term representation of a skill needs enhanced M1 representation (Karni et 
al., 1998; Ungerleider, 2002), e.g. skilled piano players have larger finger representations than unskilled (Elbert \& Flor, 1999). The involvement of the SMA on the other hand seems to be greatest in the early stages of skill acquisition (Dayan \& Cohen, 2011), whilst in the later phases, some report decreased SMA activity (Lohse et al., 2014) and some increased (Ungerleider, 2002).

Those few studies that investigate supraspinal motor dependent plasticity in SCIsubjects use locomotor training as intervention. For example, after twelve weeks of locomotor training Winchester et al. report increased activations in the sensorimotor cortical regions (S1, S2) and cerebellum in four motor incomplete tetraplegic subjects (Winchester et al., 2005). They suggest that motor improvement is related to an increase in cerebellum activity, but the small sample size makes a definite conclusion impossible.

In a case study with a C5 patient, Chisholm et al. found neuroplasticity even after a single hour of locomotor training (Chisholm, Peters, Borich, Boyd, \& Lam, 2015). Motor evoked potentials (MEPs), measured with TMS, were increased by $80 \%$ in the leg tibialis muscle and functional connectivity was increased in the right motor cortex after training.

In an interesting case study Henderson et al. reported a posterior shift, but no growth of the hand area, after massed training with complex hand movements (Henderson, Gustin, Macey, Wrigley, \& Siddall, 2011), supporting the impression that the sensory cortex is involved in compensatory functions. They demonstrate that stimulating the sensory cortex with TMS improves grip force, highlighting the close relationship between the motor areas and the sensory cortex in subjects with SCI.

The results of these studies make clear that brain organization after SCI is not static, but can be influenced by training, as is the case in healthy subjects. The nature of the influence is less clear, but motor training might induce an additional increase in activation size on the post-SCI motor cortex. However, more research is needed on this topic since the existing studies are insufficient.

\subsection{Transradial arm amputation and prosthesis control options}

The loss of a limb is a major trauma that severely affects the quality of life for victims. Out of all amputations, approximately 25\% affect the upper limbs (Esquenazi, 2004). Around 41,000 people live with a major loss of the upper limbs in the United States 
alone (Ziegler-Graham, MacKenzie, Ephraim, Travison, \& Brookmeyer, 2008). The causes for limb amputations vary world-wide and according to the political and socioeconomic standards of the country. In developing countries, insufficient treatment after trauma is the primary cause for amputation, which can be caused by accidents or armed conflicts. Although amputations due to trauma are decreasing (Meier, 2004) in the first world, diabetes and related vascular complications have become the leading causes for amputation (Esquenazi, 2004). The prevalence of diabetes is projected to nearly double in the USA in the next 30 years, hence the number of people suffering from limb loss will increase considerably (Esquenazi, 2004; Ziegler-Graham et al., 2008). Two thirds of amputees are male (Clement, Bugler, \& Oliver, 2011). Upper limb amputations are most likely to occur in the years of highest work output (60\% between 16 and 54 years) and can severely interrupt the productivity of the affected individual (Clement et al., 2011). Amputation levels of the upper limb can be divided into shoulder disarticulation, transhumeral (above elbow, Figure 4), elbow disarticulation, transradial (below elbow) and wrist disarticulation.

The human hand is an extremely complex organ that includes 22 degrees of freedom (DOF), which are controlled by 38 hand muscles (Zecca, Micera, Carrozza, \& Dario, 2002). The loss of this highly specified tool is a significant impairment which humanity has sought to remedy since the Roman Empire (Meier, 2004). The first electrically controlled arm prosthesis was developed in 1949, and later in the 1960's the first myoelectrically (with electrical potentials derived from muscles) controlled hand prostheses became commercially available. During the last three decades considerable developments have been made to improve all aspects of upper limb prostheses, such as fitting techniques and socket fabrication, which is important for a natural, daily use (Esquenazi, 2004). Progress in technology has led to new sources of power and electronic controls. 

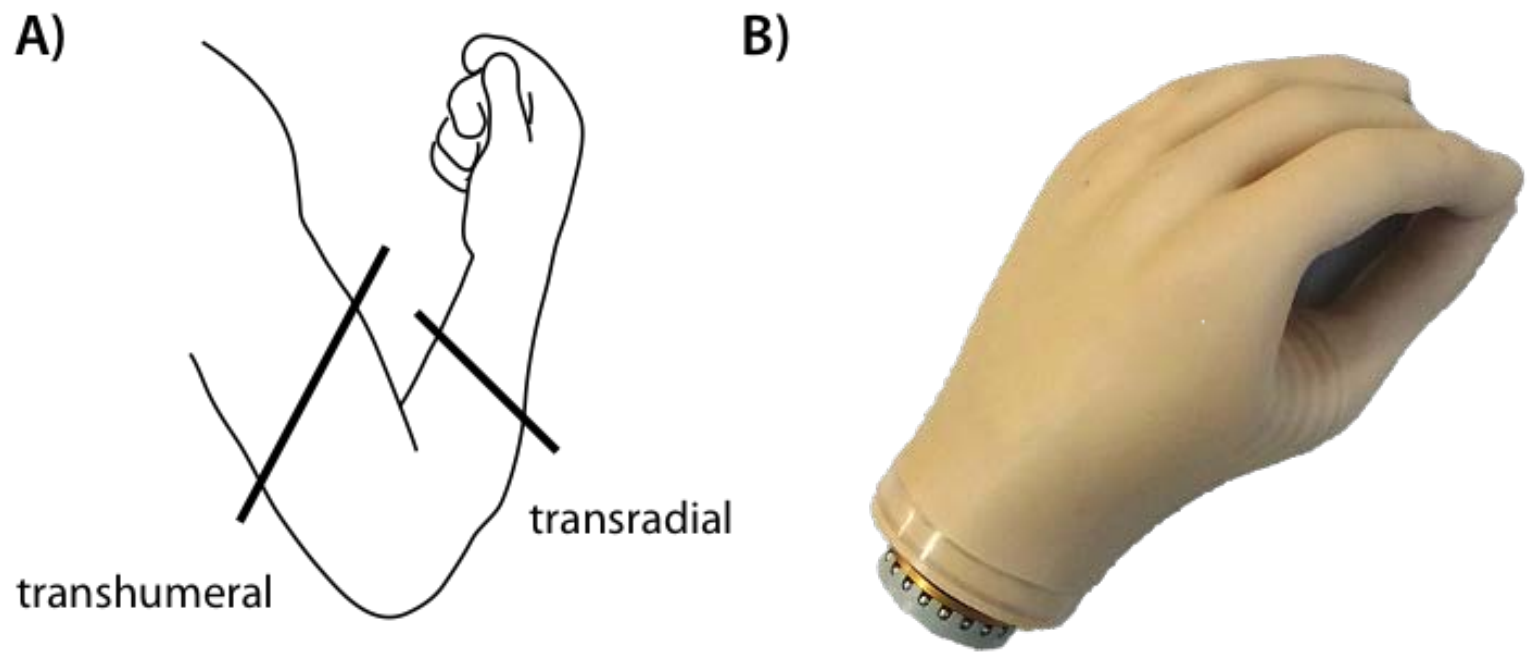

Figure 4) Arm amputation levels (A) and a common prosthetic hand (B).

Most common amputation levels of the upper limb include the transhumeral level (above the elbow) and the transradial level (below the elbow). In both cases, the loss of the hand can be compensated with a prosthetic hand (B, System Electric Hand DMC from Otto Bock, Duderstadt, Germany). This hand can be opened and closed and rotated. The prosthesis is fixed to the individual's arm with a custom made shaft, where commonly activity of the residual stump muscles is measured with surface electrodes and used as control signals for one DOF of the prosthetic hand.

Generally speaking, three types of hand prostheses are available to end-users that are amputated below the elbow. Passive prostheses mainly have aesthetic functions and can help supporting weight, but have no independent function. Body controlled prostheses use simple mechanical forces to e.g. open or close a mechanical hook. Users wear a harness around the shoulders that is connected with strings to the hook. Arm or shoulder movements stretch the strings, which in turn pull the hook open. Although the control logic was developed in the nineteenth century (Meier, 2004), it still enjoys popularity among amputees due to the simple and reliable control (Biddiss \& Chau, 2007). Nowadays, signals are amplified with linear potentiometers that can also provide proportional movement (Cipriani, Controzzi, \& Carrozza, 2011), e.g. wider movement equals stronger signals. A major drawback however is the very limited repertoire of movements available (Clement et al., 2011), which does not come close to a normally functioning hand.

External power sources, e.g. batteries, are used for externally powered prostheses. Prosthetic hands that are in clinical use typically have two DOF, i.e. opening / closing and rotation (Figure 4). The most common control system is myoelectric (Clement et al., 
2011), i.e. muscle signals are recorded with surface electrodes from the residual stump and are then used to generate the control signals. The complexity of such control signals differs widely, from digital control where simple open / close signals are produced by the presence or absence of a muscle signal, to proportional control, where the bandwidth of the muscle power signal is utilized e.g. for modulation of movement speed or different movements. This amplitude-based control is used in all commercially available myoelectric hand prostheses (N Jiang, Dosen, Muller, \& Farina, 2012), which are currently not able to control more than two degrees of freedom (DOF)(Zecca et al., 2002), whilst usually, only one DOF is controlled directly (Muzumdar, 2004). One of the most commonly used control options utilizes two DOF from two residual stump muscles. Contraction of the wrist extensor opens the prosthetic hand and contraction of the wrist flexor closes it. In order to turn the hand, a quick co-contraction of both muscles switches into the turning mode (this control is henceforth referred to as co-contraction, $\mathrm{CC}$ ). Here, flexor contraction turns the hand left and extensor contraction turns it right, with speed of movement being proportional to signal strength (Farina et al., 2014). This control is very robust and simple, but it is also slow and tiring. In another clinically established control mode, slope of signal increase determines the output action: slow contraction of the extensor opens the prosthetic hand and quick contraction turns it right (this control is henceforth referred to as slope control, SL). Conversely, slow contraction of the flexor closes the hand while a quick contraction turns it left (Farina et al., 2014). Because there is no need to switch between actuation modes, SL is quicker than CC, but it still does not allow for simultaneous control of multiple DOFs and requires a short relaxation period between two consecutive activations.

Apparently these two most widely used control modes (Muzumdar, 2004) do not satisfy prosthetic end users, with only a quarter of amputees using myoelectric prostheses (Wright, Hagen, \& Wood, 1995). A survey has reported particularly variable rejection rates for electric devices that range from 0\% to 75\% (Biddiss \& Chau, 2007). Despite considerable improvements in technology, material and fitting, there seem to be no indications of a decrease in rejection rate. The reasons for rejection range from reliability of signal generation, unstable electrodes and limited wrist movement (Atkins, Heard, \& Donovan, 1996). The daily use of arm prostheses is "notoriously challenging" (Biddiss \& Chau, 2007), hence in an estimated sub-group of $16 \%$ - 47\% the active prostheses are used as passive prostheses, without making full use of their capabilities. 
Necessary improvements in prosthetic devices include coordinated movement of multiple joints, i.e. several DOF, and greater intuitiveness of the control (Engdahl et al., 2015).

The clinically available control methods are not able to match the complexity of new hand prostheses with several degrees of freedom, which have been developed over the last few years in order to replicate some of the original complex hand functions (Atzori \& Muller, 2015; Belter, Segil, Dollar, \& Weir, 2013; Cipriani et al., 2011; Clement et al., 2011; Zecca et al., 2002). For simultaneous control over several DOF, pattern recognition has received considerable attention from the scientific community. This approach is based on the assumption that patterns of muscle activation can be distinguished, repeated and subsequently used as a command for a pre-specified movement ( $\mathrm{N}$ Jiang et al., 2012). Classification algorithms identify patterns from the information of several electrodes (Englehart, Hudgins, \& Parker, 2001; J. M. M. Hahne, Graimann, \& Muller, 2012; Pistohl, Cipriani, Jackson, \& Nazarpour, 2013). In their original form, classification based approaches do not allow for controlling the speed or the grip-force of the prostheses as do the conventional approaches. Therefore, they are often combined with a force-estimation based on EMG-amplitude (Zecca et al., 2002). As all functions can be accessed directly, pattern recognition is more intuitive and faster than switching the active DOF as in the clinically established control. All of these controls however suffer from the lack of simultaneity: only one DOF can be addressed at one time. In pattern recognition, complex movements still have to be split and carried out sequentially (Farina et al., 2014). To solve this problem, combined classes have been introduced as well as regression-based approaches that directly allow for controlling multiple DOF at the same time (J. Hahne, Dahne, Hwang, Muller, \& Parra, 2015; J. M. M. Hahne et al., 2012; N Jiang et al., 2012; Scheme \& Englehart, 2011). Pattern control is reported consistently to have high classification accuracy ( $>90 \%$ ) and could answer to the requirements of more sophisticated control for complex hand movements. However, the clinical impact has been virtually zero until now. The major obstacle is the robustness, which until now can only be guaranteed in laboratory settings (Farina et al., 2014). Another disadvantage is that signal transfer is not continuous, as natural movements are, but can only activate pre-defined sets of activities. Proportional control is only possible post-hoc, since it reduces correct classification (N Jiang et al., 2012). Other issues include adaptation to electrode displacements (Hargrove, Englehart, \& 
Hudgins, 2008; Young, Hargrove, Kuiken, \& others, 2011), variation of the arm position (Fougner, Scheme, Chan, Englehart, \& Stavdahl, 2011; Ning Jiang, Muceli, Graimann, \& Farina, 2013), changes in skin conditions (N Jiang et al., 2012), transfer between training and application (Vidovic et al., 2016) or difficulties in subject training (Powell \& Thakor, 2013). For these reasons, pattern recognition has not yet left the laboratory and the reliability in real-world conditions is limited (N Jiang et al., 2012).

Given these problems, there have been calls for new methods that enable simultaneous and proportional control of several DOF (N Jiang et al., 2012). Several propositions have used completely different approaches to control additional DOFs. For example, switches on a foot inlay can be used to control different pre-set grasps more quickly than a pure EMG-control (Carrozza et al., 2007). Likewise, vocal commands with a throat microphone (Mainardi \& Davalli, 2007) and prosthetic control with shoulder movement (Losier, Englehart, \& Hudgins, 2011) have been shown to outperform simple EMG control. Although these systems have shown superior performance, they still interfere with other activities. Limitations in e.g. free movement (foot inlay, shoulder movement) or communication (vocal commands) are likely to be seen as too restrictive for acceptance by users. Moreover, simultaneous and proportional control over free hand movements is not possible with these approaches.

More sophisticated computer-based approaches include the cognitive vision system, where a camera-based system identifies objects and chooses the adequate grasp type autonomously (Dosen et al., 2010). Brain computer interfaces (BCI) have also been proposed for prosthesis control (Lebedev \& Nicolelis, 2006). In an impressive animal study, it was shown that monkeys can learn to control a prosthetic arm for self-feeding with their motor cortex activity, independently of executed movements (Velliste et al., 2008). However, in order to become viable control alternatives, BCI need to improve information transfer rates and robustness (Wolpaw et al., 2002).

Other approaches use surgical interventions to achieve alternative control strategies. Targeted muscle re-innervation (TMR) surgically redirects the arm nerves to the chest, where surface EMG can detect volitional activation for prosthesis control (Johansen et al., 2016). This procedure has yielded promising results, but is considered too invasive for below elbow amputees and needs further technical development to achieve proportional and robust prosthetic control (Atzori \& Muller, 2015). In a completely 
different approach, using an osseo-magnetic link, a small magnet is implanted in the stump, whilst sensors in the socket of the prosthesis can detect even small movements of the bone via changes in the magnetic field and can control four degrees of freedom (Rouse, Nahlik, Peshkin, \& Kuiken, 2011).

Despite being promising alternative directions for the rehabilitation care of amputees, none of these academic approaches to prosthetic control have left the laboratory, and in some cases no data from human trials has been presented yet. In order to overcome the gap between research and clinical implementation and increase acceptance among users, robust proportional and simultaneous control systems are needed (N Jiang et al., 2012). The independent control of two degrees of freedom is a challenge that needs to be solved in order to achieve this goal.

\subsection{The auricular muscles in the context of human-machine interfaces}

Humans possess nine muscles per ear with six intrinsic and three extrinsic muscles (Gray, 1918). The most prominent extrinsic muscles are the m. auricularis superior and posterior (PAM), located above and behind the pinna (Figure 5). Darwin suggested that they might once have served for sound localization, as with other mammals, and have lost that function during evolution (Darwin, 1859). Due to the lack of function in humans, the auricular muscles have received little attention from the scientific community. In the 1960s, the post-auricular reflex was discovered, a bilateral, soundevoked contraction of the posterior auricular muscle (Kiang, Crist, French, \& Edwards, 1963). Together with the oculo-auricular phenomenon (Wilson, 1908), an automatic auricular muscle contraction with maximum lateral eye movements, it rendered evidence for a sound-location origin of these muscles that today are in a "hibernate" mode and lack a dedicated function. 


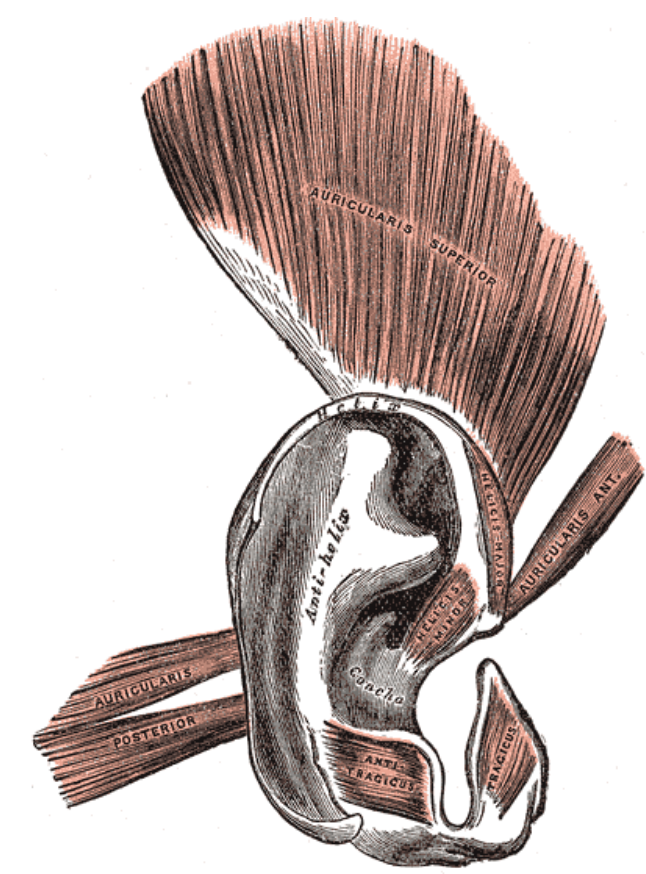

Figure 5) Anatomy of the human auricular muscles.

There are 6 intrinsic muscles on the pinna and three extrinsic muscles, located anteriorly, superiorly and posteriorly from the ear. For the ACS, we make use of the isolated position of the posterior auricular muscle on the scalp, which ensures minimal crosstalk form other muscles. (Taken from https://commons.wikimedia.org/wiki/Category:Auricular_muscles\#/media/File:Gray906.png, Retrieved 08.12.2017.)

In the context of the ACS this lack of function becomes an advantage, because unlike other control systems the ACS does not interfere with daily activities or communication (e.g. tongue control) or suffers from the Midas Touch problem, i.e. distinguishing between intended and unintended commands (e.g. visual control (Barea, Boquete, Mazo, \& Lopez, 2002)).

Regarding voluntary activation of the auricular muscles, conflicting evidence has been reported. While one study reports voluntary activation of the superior and posterior auricular muscles (Berzin \& Fortinguerra, 1993), another study denies this and regards them as rudimentary without any function (Serra, Tugnoli, Cristofori, Eleopra, \& De Grandis, 1986).

Interestingly, the auricular muscles have however been chosen in two studies to investigate human-machine interfaces (HMI). In a study from Perez-Maldonado et al., subjects could learn to control the different bandwidths of activity of the superior auricular muscle, thereby producing 2 DOF from a single-site EMG (Perez-Maldonado, Wexler, \& Joshi, 2010). Larson et al. chose the posterior auricular muscles to investigate different feedback paradigms for learning (Larson, Terry, \& Stepp, 2012). Subjects were 
able to produce voluntary bilateral signals with their posterior auricular muscles, first controlling a cursor target with visual and auditory feedback, and then with only auditory feedback. However, they concluded that other muscles might be more functional for HMI, since the posterior auricular muscle (PAM) is vestigial and "it is precisely its vestigial nature that makes it difficult to learn to control compared to other muscles.".

There are several reasons why the active control over these muscles would mean a breakthrough. Firstly, their lack of function means there is no interference with other mimic activities, including communication. Secondly, these muscles exist in all humans and, if functional, they represent an enormous unused potential. Since they are controlled by the facial nerve, their functionality still exists even in high-level tetraplegia. Voluntary modulation of the auricular muscles would disclose new functional possibilities for these muscles, e.g. in the context of HMI.

These factors make the auricular muscles an ideal option for myoelectric control. In addition, the isolated position of the posterior auricular muscle (PAM) ensures minimal crosstalk. The bigger superior auricular muscle for example is located above the temporalis muscle, which would produce considerable artifacts while e.g. chewing. Good accessibility of the PAM renders it particularly suitable for a myoelectric control interface. However, due to the scarce literature available about voluntary auricular activation, there are several still unknown characteristics of these muscles, which shall be addressed in this thesis. In order to use the auricular muscles for generation of control signals, it is necessary discover how voluntary activation of these muscles can be learned and trained. It has been shown that bilateral activation was possible in some subjects (Larson et al., 2012). However, it is unknown whether complex activation patterns (e.g. different strengths, unilateral activation) can be achieved. If this was the case, signals from the bilateral PAM could provide independent control over two DOF (left side activation, right side activation) in the context of human-machine interface and could generate signals for a third channel (co-contraction of both sides). An advantage of an EMG-based control system is that signal strength can be proportional to the muscle strength. Precise modulation of PAM-strength would allow for direct control of the speed of the output action besides simple on-off, e.g. speed of the wheelchair or prosthetic hand action. In addition, the continuous signal generation of EMG would 
permit for online control of the output device (e.g. small corrections of the output actions), thus potentially increasing the precision of the device.

\subsection{Aims of this thesis}

Assistive devices are essential to improve participation and life quality of affected individuals, such as individuals with tetraplegia or arm amputation. We have established that existing control options present different shortcomings. Difficult and cumbersome signal generation is a problem in both wheelchair and prosthesis control. Technical issues as well as interference with other activities have reduced the impact of proposed scientific control options on clinical implementation so far.

To address some of the drawbacks of available control option, during the course of this thesis we developed an alternative control method, which uses the myoelectric potential of the posterior auricular muscles. The auricular control system (ACS) could positively impact those individuals with tetraplegia who are afflicted with reduced mobility due to inadequate control options. It also has the potential to improve hand-prosthesis control and thus increase acceptance rate amongst prosthesis users. By providing control over assistive devices, it could ultimately improve the life quality of end-users.

The first study examined the prerequisites for the ACS, i.e. if subjects could learn voluntary and unilateral activation of the PAM. Importantly, it represents the first proofof-principle of the functionality of the ACS for wheelchair control. To this aim, ten ablebodied subjects and two subjects with tetraplegia trained voluntary activation of bilateral PAM with visual feedback and computer games for four consecutive days and steered a wheelchair using only the ACS on the fifth day.

The aim of the second study was a clinical application of the ACS to hand prosthesis control, in order to investigate whether the ACS could improve performance of available prosthesis control methods. A combination with an established forearm control allowed for simultaneous, independent control over two DOF in a prosthetic hand. This hybrid control was tested in 10 able-bodied subjects and one subject with transradial amputation and its performance was compared with two clinically established control modes. 
The aim of the third study was to test functionality of the ACS in a clinical sample of individuals with tetraplegia, and hence to investigate the effects of motor training on the post-SCI brain. The specific brain reorganization in these subjects allows investigation of neuronal plasticity from a different, yet uninvestigated perspective: how do unoccupied areas of the brain respond to new tasks? Targeting the brain reorganization in SCI subjects could have important implications for therapeutic care. This study presents the results of twelve days of ACS wheelchair training in nine subjects with high-level tetraplegia. To investigate the effects of motor training, we mapped the motor cortex of these subjects before and after ACS training, using transcranial magnetic stimulation (TMS) and functional magnetic resonance imaging (fMRI).

In combination, the aim of this thesis is the development of a new control method for assistive devices, while providing further information about the characteristics of the human auricular muscles. The functionality of the ACS will be examined in two prototypical clinical indication fields, namely hand prosthesis and wheelchair control. The resulting motor training permits an investigation of the mechanisms of activitydependent neuronal plasticity in individuals with tetraplegia, which are still unclear. 


\section{Steer by ear: proof of principle of the ACS for wheelchair control}

As presented in the introductory section, mobility is essential for the quality of life of individuals with tetraplegia (Craig et al., 2009; Middleton et al., 2007). Nevertheless, the options for wheelchair control for these individuals present major drawbacks. Simple activities of daily living become an obstacle for 50\% of individuals with tetraplegia, when using the available systems (Fehr et al., 2000). In addition, depending on the lesion level, individuals with tetraplegia might not have the physical requirements for using the available controls, e.g. activating neck muscles in order to use chin or head controls (Arshak et al., 2006). Alternative control options are promising, but interfere heavily with other activities.

Due to their isolated position on the scalp and lack of function, interference is not a problem with the posterior auricular muscles. Other characteristics presented in the introduction, such as availability in high level SCI, speak in favor of these muscles as a source for steering signals. There is very little evidence about the nature of the auricular muscles; namely if they can be voluntarily controlled, whether this ability can be learned and whether they can be activated unilaterally. These pre-requisites for the auricular control system were investigated over four days computer feedback training with ten able-bodied subjects and two with tetraplegia. Ultimately, the application of the ACS for powered wheelchair driving was tested on the fifth day in order to ascertain whether the PAM can generate precise control signals.

The following chapter 2 corresponds largely to the article "Steer by ear: myoelectric auricular control of powered wheelchairs for individuals with spinal cord injuries" published in Restorative Neuroscience and Neurorehabilitation (Schmalfuss et al., 2016). Reprinted with permission from IOS Press. 


\subsection{Materials and Methods}

\subsubsection{EMG recording and signal transmission in the ACS}

To implement an intuitive wheelchair steering system, EMGs were recorded from both left and right PAMs. For myoelectric control interfaces, usually surface electrodes are attached to the skin that record action potentials from activated motor units below the electrode (Asghari Oskoei \& Hu, 2007). However, a major problem with the use of surface electrodes is the unstable signal quality due to external influences such as sweat or varying skin impedance (Zecca et al., 2002). An alternative is subcutaneous electrodes. They can improve signal stability, but also have the advantage of higher specificity. Due to the location inside the muscle, they are able to selectively record the activity of single motor units and thus reduce the risk of artifacts from adjacent muscles.

We hence recorded PAM EMG with subcutaneous fine-wire electrodes that were custom made from stainless steel wires $(12 \mathrm{~cm}$ long, diameter: $50 \mu \mathrm{m}$, California Fine Wire Company, Grover Beach, California, U.S., Figure 6). The isolation of the electrodes was removed at both ends and along one $\mathrm{cm}$ in the middle.

For subcutaneous electrode placement, electrodes were inserted into a hollow needle that passed through the skin twice. The end of the electrode was held while the needle was pulled out. Next, the non-isolated fine-wire middle was placed under the skin. In this way, an active electrode was placed over the PAM and a reference electrode was placed in the middle of the pinna on each side. The electrodes were fixed and covered with a plaster when they were not used. Myoelectric activity was recorded as a differential signal between the electrode over the PAM and that over the pinna. An $\mathrm{Ag} / \mathrm{AgCl}_{2}$ sintered earlobe clip electrode (Gelimed, Bad Segeberg, Germany) was used on each side as a reference (Figure 6). For signal transmission, the ends of the electrodes were connected to self-built amplifiers that were fixed on a goggle frame. Here, the EMG signals were amplified (gain = 1000), band-pass filtered (4th order Butterworth filter, 20-1,000 $\mathrm{Hz}$ ) and digitized (sampling frequency $2 \mathrm{kHz}$ ). The data was then rectified, low-pass filtered and down-sampled to $125 \mathrm{~Hz}$. A ZigBee interface was used to send the raw EMG-data to a specially developed software (Bartschat et al., 2012). 


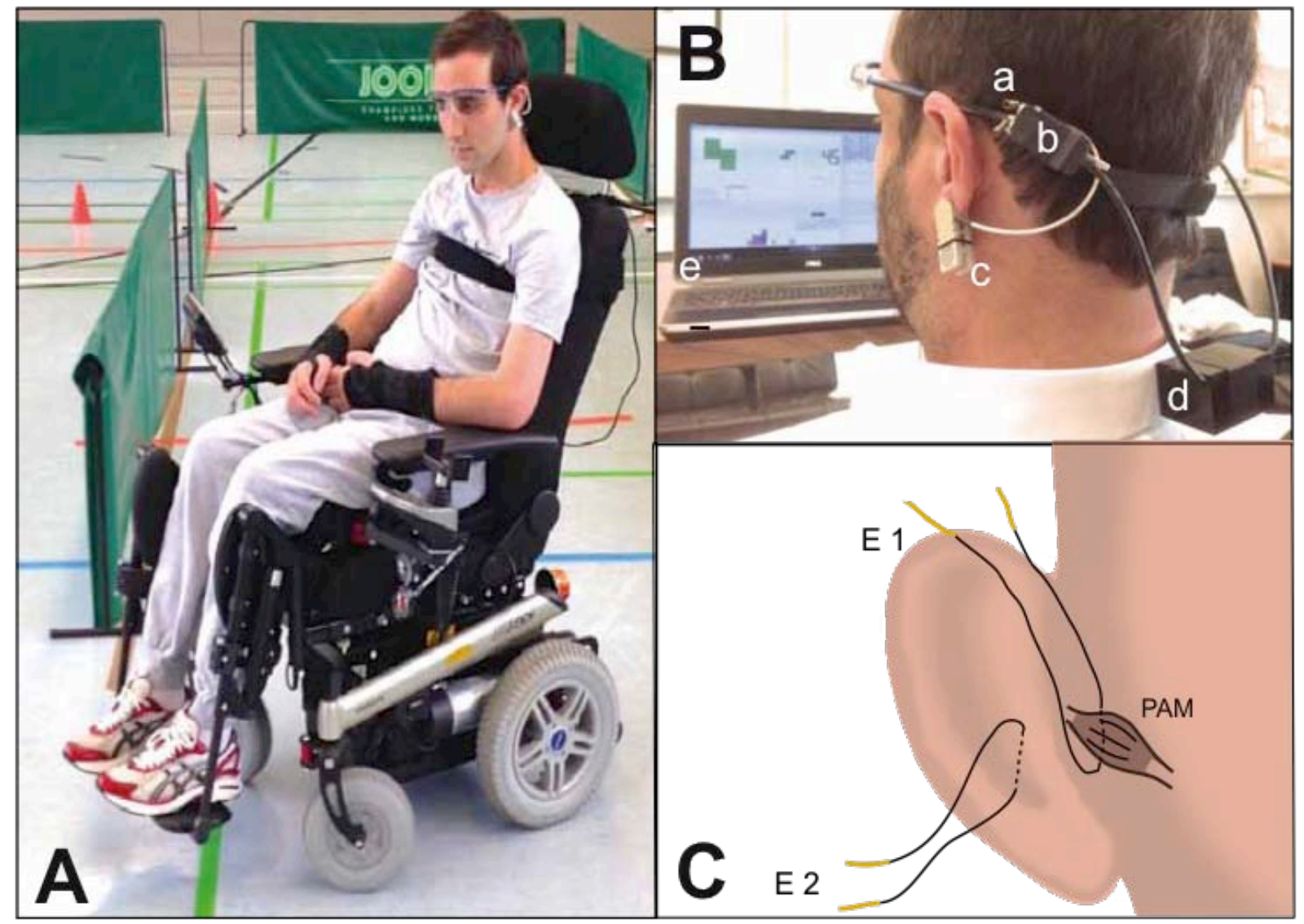

Figure 6) Setup of the auricular control system (ACS)

Subject T1 (A) driving through the real obstacle course with a powered wheelchair using the ACS. The computer is fixed in the back of the wheelchair. The EMG setup (B) held by a goggles frame with fine wire electrodes that are attached to a metal spring (a) connected to the amplifier (b). Ear clips (c) are used for grounding. A wireless transmitter (d) sends the signal to a receiver, which forwards it to the software (e). The fine-wire electrodes (C) are $12 \mathrm{~cm}$ long and have three de-insulated regions (marked yellow) to ensure conductivity. The middle part is placed bellow the skin and both ends are attached to the amplifier. The active electrode (E1) is inserted over the PAM, the reference electrode (E2) over the pinna.

\subsubsection{Generation of control signals in the ACS}

In the software, the raw, 10-bit digitized EMG signals $x_{i}[k] \in\left[0,2^{10}-1\right] \mathrm{x}_{\mathrm{i}}[\mathrm{k}] \in$ $\left[0,2^{10}-1\right]$ (Figure 7 A) were mean-adjusted and rectified in accordance with

$\mathrm{x}_{\mathrm{i}, \mathrm{r}}[\mathrm{k}]=2^{*}\left|\mathrm{x}_{\mathrm{i}}[\mathrm{k}]-\mathrm{x}_{\mathrm{i}, \text { mean }}\right| \in\left[0,2^{10}-1\right]$

where $x_{i, m e a n}$ is the mean value found in the calibration (see below). Subsequently, the resulting signals are low-pass-filtered (Root-Mean-Square Filter with m=19 and InfiniteImpulse-Response Filter with $\mathrm{a}=0.9$ ) according to

$\mathrm{x}_{\mathrm{i}, \mathrm{f}}[\mathrm{k}]=\mathrm{a}^{*} \mathrm{x}_{\mathrm{i}, \mathrm{f}}[\mathrm{k}-1]+(1-\mathrm{a}) * \sqrt{\frac{1}{\mathrm{~m}+1} * \sum_{\mathrm{j}=0}^{\mathrm{m}} \mathrm{x}_{\mathrm{i}, \mathrm{r}}^{2}[\mathrm{k}-\mathrm{j}]} \in\left[0,2^{10}-1\right]$ 
Eventually, the resulting signals are normalized to a range between 0 (minimum signal amplitude) and 1 (maximum signal amplitude, see Figure $7 \mathrm{~A}$ ) according to

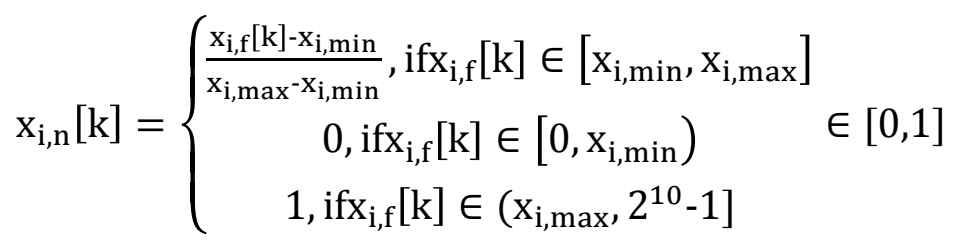

where $x_{i, \max } \mathrm{x}_{\mathrm{i} \text {,max }}$ and $\mathrm{x}_{\mathrm{i} \text {,min }}$ are the maximum and minimum values respectively found in the calibration.

The process of calibration was done by a fully automatic routine, which took the average mean, maximum and minimum of three contractions of each PAM side. This way, the signal strength was matched to the individuals' muscle strength. The minimum threshold was adjusted manually in order to minimize the risk of false-positive commands.

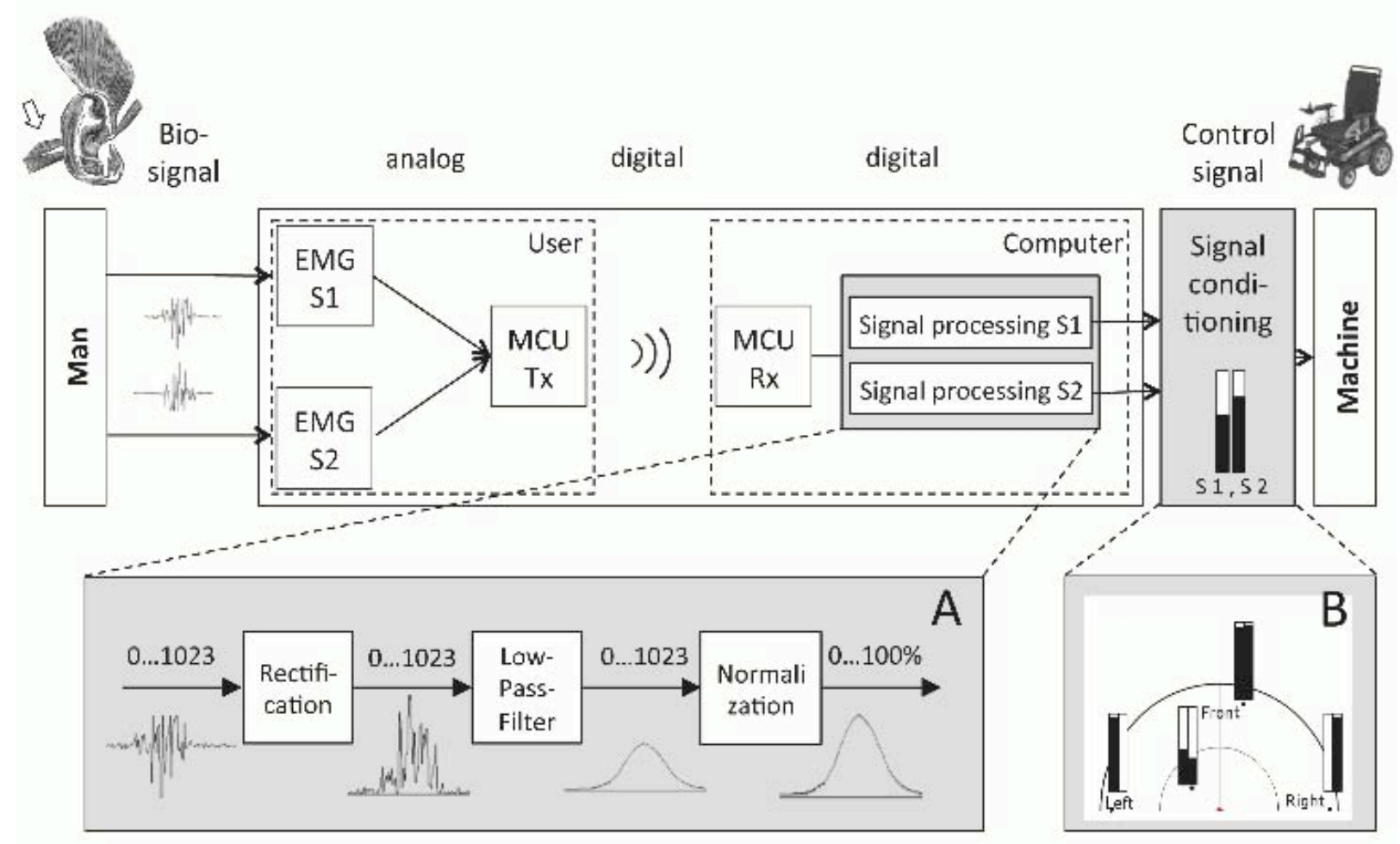

\section{Figure 7) Diagram of signal processing in the ACS.}

The raw EMG-signals from the right and left PAM (EMG S1 and EMG S2) are sent from the Microcontroller Unit (MCU) Tx (Sender) with a wireless ZigBee protocol to the receiver (MCU Rx) attached to the computer. The software rectifies, low-pass filters and normalizes the signals $(\mathrm{A})$ and conditions them into control signals (B). The output signals are transmitted to the machine, in our case the wheelchair. If both signals are equally strong, the wheelchair is propelled forward. If one signal is stronger than the other, a turn is initiated with a simultaneous forward movement depending on the strength of the co-activation. 


\subsubsection{Generation of wheelchair driving commands}

For electric wheelchair driving (B 600, Otto Bock, Duderstadt, Germany), the computer with the ACS interface was connected to the external joystick interface. Stronger left PAM-contraction produced a left turn, stronger right contraction produced a right turn and a co-contraction propelled the wheelchair straightforward. The output signal for the wheelchair consisted of a rotational and a translational signal. The rotational signal was calculated by the different activation of both sides (e.g. left side stronger: turn left). Here, the bilateral calibration helped to identify a rotational signal, if lateral selectivity was not that pronounced. The translational signal was calculated by the mean contraction strength of both sides (both sides full contraction: translational signal $(1.0+1.0) / 2=1.0$, wheelchair drives straight ahead). This way, a full activation of only the left side meant a rotational signal of 1.0 and a translational signal of $0.5((1.0+0) / 2=0.5)$, i.e. the wheelchair made a left-turn with a slight acceleration. In order to rotate on the spot without translation, a half activation of only one PAM was thus necessary. Steering signals were proportional and continuous; the participants could therefore e.g. control the speed of the wheelchair or the sharpness of turns in an online manner.

The wheelchair stopped when the signal input stopped, when the off-button was pushed manually or when the principle investigator activated a wireless switch. The update rate of the wheelchair control signal was the same as the sampling rate of the EMG $(125 \mathrm{~Hz})$. Speed depended on the duration of the co-activation, with a slight delay due to the lowpass-filtering. The maximum velocity of the wheelchair was preset to $5.2 \mathrm{~km} / \mathrm{h}$ in the wheelchair configuration.

\subsubsection{Proof-of-principle of the ACS}

The research protocol was approved by the ethics committee of the University of Göttingen and was in accordance with the latest version of the Declaration of Helsinki. Ten able-bodied subjects and two subjects with tetraplegia participated in the study. Written informed consent was obtained from each subject or from an independent representative. The able-bodied subjects (mean age: $24.0 \pm 1.8$ y; 4 female, 6 male) were right-handed according to the Edinburgh Inventory of Handedness (Oldfield, 1971). 
Subjects categorized themselves as "ear-wigglers" (EW, able to activate PAM, 5 subjects) or "non-ear-wigglers" (NEW, not able to activate PAM, 5 subjects).

Four patients from the patient database of the Spinal Cord Injury Center of Heidelberg University Hospital met the inclusion criteria (tetraplegia caused by trauma, neurological level of injury C8 or higher, exclusion criteria: dependence on artificial ventilator, former episodes of epilepsy). Two of the four dropped out for medical and technical reasons, and two completed the study. Subject T1 (male, 23 y) has complete motor and sensory tetraplegia (American Spinal Injury Association (ASIA) Impairment Scale A (Kirshblum et al., 2011)) with a neurological level of injury at C5 due to a trauma seven months before inclusion in the study. Subject T2 (male, 53 y) suffers from incomplete sensory and motor tetraplegia (ASIA Impairment Scale C) with a neurological level of C3 after a fall five months before study onset. Both were hospitalized for primary rehabilitation at the time they were included in the study and were on medication with spasmolytic drugs. Subject T1 classified himself as an "earwiggler"; subject T2 as a "non-ear-wiggler".

The study design consisted of daily training sessions (1 hour each) on four consecutive days and $30 \mathrm{~min}$ of wheelchair driving in a fifth session on day 5. For each session, the subjects were equipped with the wireless EMG recording system (Figure 6). For the first 20 minutes, the ability to activate the PAM was measured (duration 20 minutes) using eight performance criteria at each session (session 1 - session 4) and before and after driving the wheelchair (session 5a, session 5b). For the remaining 40 minutes of each 1 hr. session, subjects played four computer games solely with PAM activation (see Supp. Fig. 1 and Supp. Fig. 2). Because of general physical and mental exhaustion after the wheelchair drive, the testing was skipped in session $5 \mathrm{~b}$ for tetraplegic subject $\mathrm{T} 2$ as well as the contraction duration measurement for subject $\mathrm{T} 1$. In the last session $5 \mathrm{~b}$, subjects steered an electric wheelchair by means of signals generated by activating their PAMs. Evaluation questionnaires were completed before and after the study.

\subsubsection{Training of voluntary control over the PAM}

The primary goal of the training procedure was to increase the ability to activate both PAMs in order to be able to use the ACS. In the first session, basic control over PAM 
activation was established with visual feedback on a computer screen, where the height of two bars indicated the strength of the normalized EMG activity of both PAMs.

In the successive training sessions, participants chose from four PAM-controlled computer games. These games were especially designed to keep subjects motivated, train lateralized PAM activation and to precisely modify the control signal. In a car racing game (Supp. Fig. 1), the subjects had to keep a virtual car on a moving track with either left or right PAM contractions. In the well-known game Tetris (Figure 8 B), the subjects had to complete lines of blocks by moving the pieces to the left (left contraction) or to the right (right contraction), or by rotating them (co-contraction) in order to reach a higher level with increased speed. The remaining two games, coin collection and virtual obstacle course, trained more complex driving skills with a virtual wheelchair seen in a bird's eye view. Here, the subjects had to either collect 10 coins that appeared successively (Supp. Fig. 2) or navigate through a simple virtual obstacle course with up to four turns. The virtual wheelchair navigation mode was the same as in powered wheelchair driving.

\subsubsection{Testing the performance of the ACS}

To evaluate the effect of training on the participants' ability to voluntarily activate their PAMs, eight performance criteria were defined as the main training outcome measures. They represented important signal modalities that directly influence the quality of the ACS. Each outcome measure was tested for the left and right ear separately. The final result was calculated as the mean performance of three repetitions. First, signal generation speed was tested as reaction times (RT) for contraction and relaxation (RT, Supp. Fig. 3, milliseconds $=\mathrm{ms}$ ). RT for contraction was defined as the time between the appearance of a visual cue (a red square) to contract and the point at which the normalized EMG signal reached $60 \%$ of the subjects' normalized maximum strength, while RT for relaxation was the time between disappearance of the cue and the time point at which the EMG activity went below $20 \%$ of the subjects' normalized maximum strength (empty fore-period of the visual cue $3 \mathrm{~s} \pm 1 \mathrm{~s}$ )). The maximum contraction repetition rate of the PAMs (Figure $8 \mathrm{~A}$, below called maximum contraction rate) was then tested as a measure of the ACS' maximum repetition rate of binary control signals. The rate of contractions followed by relaxations in a $10 \mathrm{~s}$ period was measured in Hertz 
(Hz, e.g. $1.5 \mathrm{~Hz}=15$ contractions in $10 \mathrm{~s}$ ). In order to get an impression of the endurance of the muscle, which is important when producing longer steering signals, we tested maximum duration of contraction (Supp. Fig. 4). The maximum time was $100 \mathrm{~s}$; the counting was stopped with the first detected relaxation $(<20 \%$ of normalized maximum signal). In graded PAM activity (Supp. Fig. 5), we tested precision of muscle control and requested the participants to hold the muscle activation at a predefined level between $20 \%$ and $70 \%$ of the normalized maximum level for $10 \mathrm{~s}$. The outcome here is the percent of the trial duration that the subject was able to hold the correct activation between 20 and 70\%. In order to turn the wheelchair using the ACS, lateralized activation is required. The subjects were hence instructed to selectively activate one side for 3 s (Supp. Fig. 6). Correct activation in this task was defined as the average activation strength of one PAM over a 10 s period. However, since unilateral activation was required, this score was diminished in case of an involuntary co-activation of the other PAM. The unintended contraction-strength was then subtracted from the correct one, so that the final score could only reach $100 \%$ if a perfect unilateral activation with no co-activation of the other PAM was achieved.

Finally, the ability to drive a wheelchair was evaluated with a single run of a virtual wheelchair through a virtual obstacle course in order to avoid a risk for unpracticed wheelchair users. Performance was measured as the length of the path driven (pixel) and the time the subject required to complete the course (seconds). 

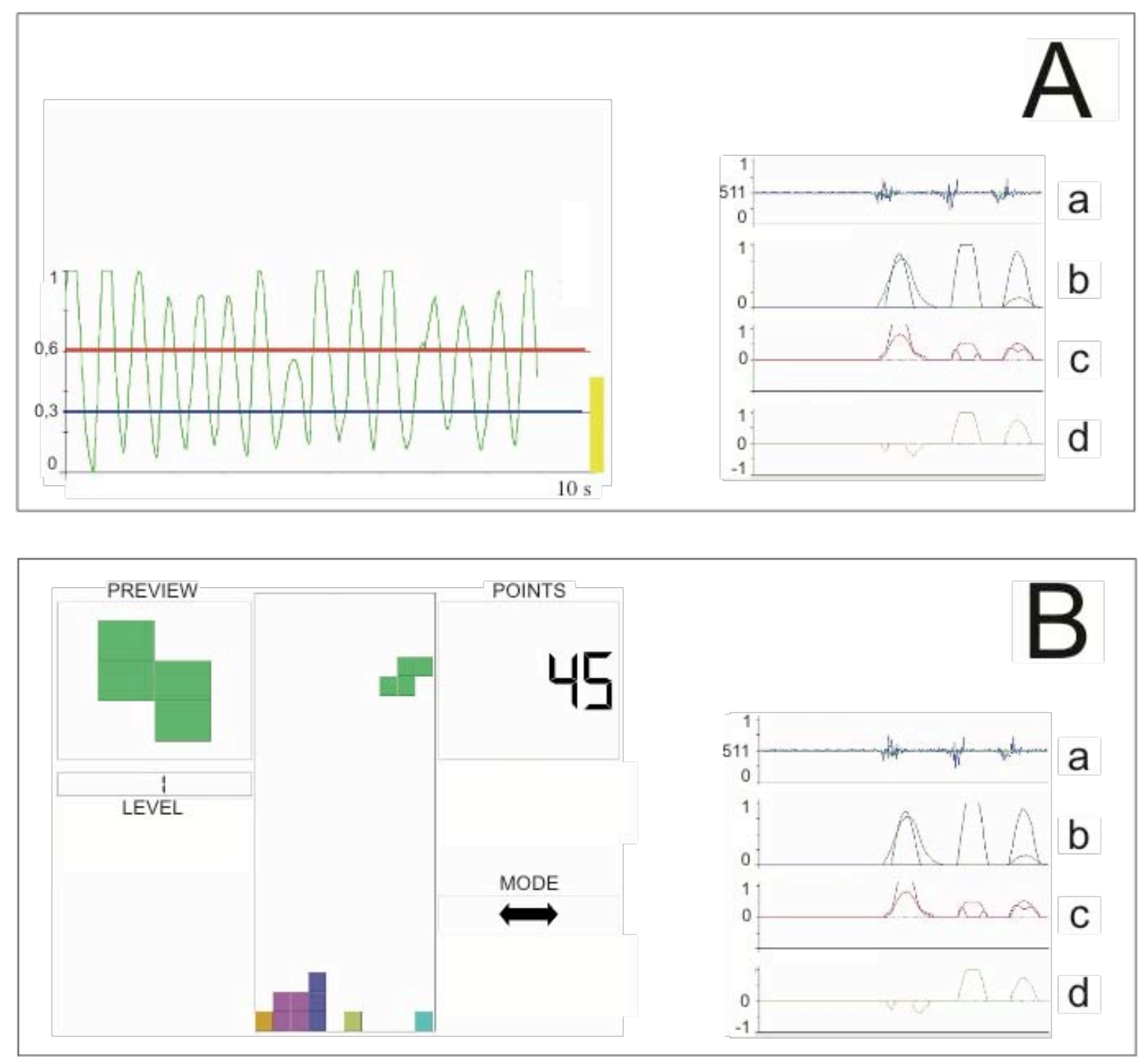

Figure 8) Signal processing in the user interface.

The software converts the raw EMG signals (a, digitized values, mean 511) into normalized signals (b, 0-1) and control signals (c, translational signal, $d$, rotational signal -1 is left, +1 is right). The normalized values (b) are used for signals during computer tasks. For example, in the performance test for the maximum contraction rate (A), subjects were told to fully contract and relax their PAM as often as possible during 10 s. A complete contraction was counted when the EMG activation passed the upper threshold $(60 \%$, red line) and subsequently went below the lower threshold (20\%, blue line). This subject managed 13 complete contractions $(1.3 \mathrm{~Hz}$ ). During training, subjects played e.g. Tetris (B) with their PAM signals. A right contraction moves the piece to the right, a left contraction to the left and a co-contraction turns it.

\subsubsection{Powered wheelchair driving with the ACS}

In the fifth session (30 min) after four days of training, the subjects steered an electric wheelchair (B 260, Otto Bock, Duderstadt, Germany) with the ACS connected to its external joystick interface. Control signals were right PAM contraction - right turn, left contraction - left turn, co-contraction - straightforward, no signal or manual use of the 
off-button - stop. The subjects first had five minutes to get accustomed to the wheelchair control and were then requested to drive straight ahead for four meters and turn $180^{\circ}$ to the left and then to the right.

Subjects with tetraplegia were then instructed to drive through a real obstacle course that was analogous in its proportions (side length of $10 \mathrm{~m}$ ) to the virtual obstacle course used in the performance criteria (Figure 9). Three independent observers measured completion time and number of collisions with the sidewalls. In case of a collision, the stopwatches were paused until the subjects were manually set back on track. For emergency stops, the principal investigator had a wireless control. The performance was recorded on video and the driving paths were later digitized offline into the virtual obstacle course template.

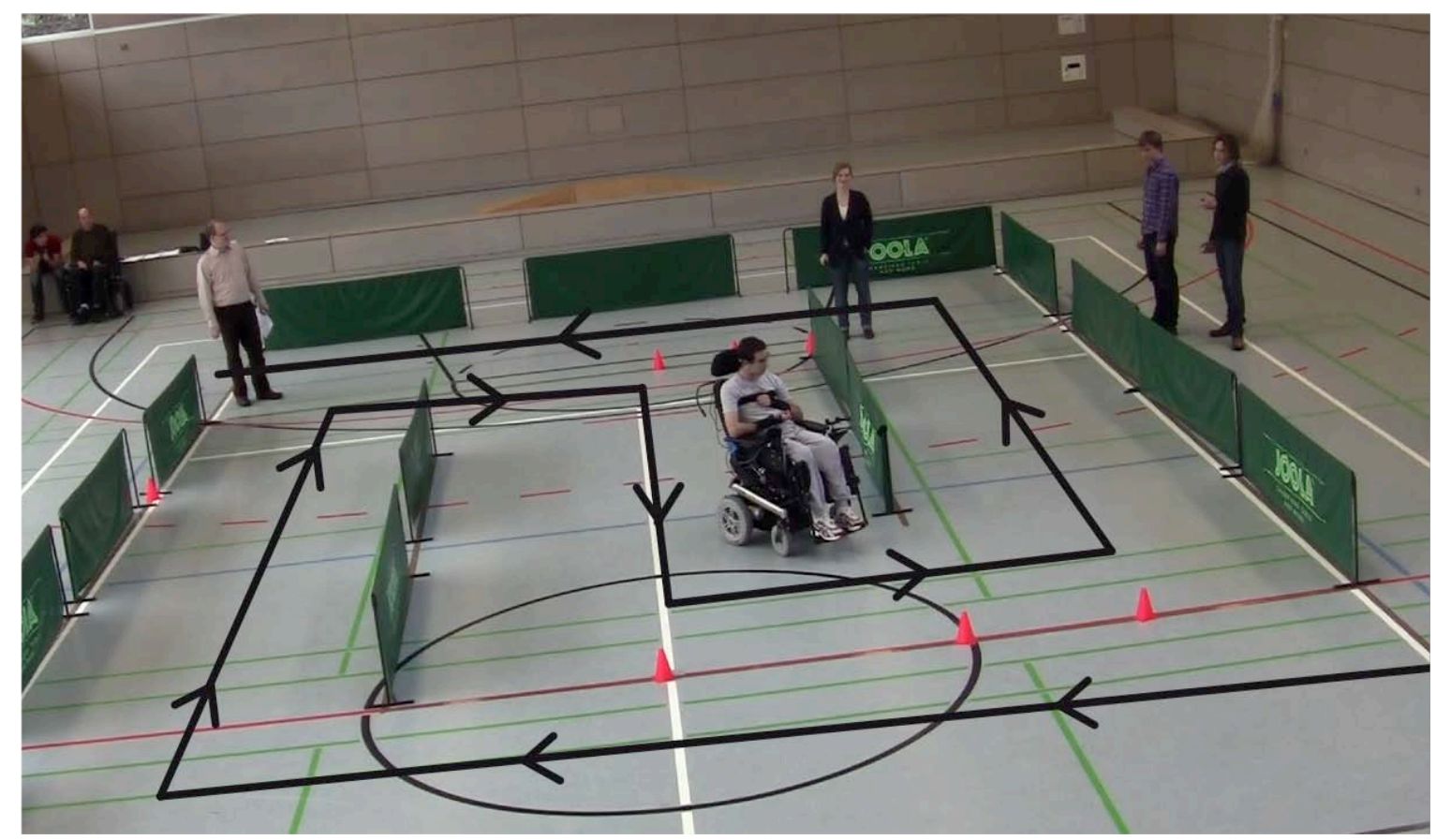

Figure 9) The real obstacle course for testing the performance of the ACS in subjects with tetraplegia.

Aerial view of the obstacle course while subject T1 completes it (driving direction marked with black arrows). The longer sides were approximately $10 \mathrm{~m}$ long, the short side $6 \mathrm{~m}$. An ideal path was ca. $23 \mathrm{~m}$ long and included three $90^{\circ}$ right turns and three left turns. Time was measured and the performance was filmed and manually digitalized for evaluation of the driving path.

\subsubsection{User evaluation of the ACS}

Before the first and after the last session all subjects completed a questionnaire about their ability to activate PAMs. They were asked to rate several questions on a four-point 
scale ranging from 1 (= not at all), 2 (= a little), 3 (= moderate), to 4 (= a lot), including overall ability to "wiggle the ears", self-rated concentration, training effort, difficulty of unilateral PAM activation (for each ear separately) and necessary co-contraction of other facial muscles to generally activate PAMs. A second questionnaire evaluated the training characteristics and contained rating items regarding effectiveness and required effort for the computer training. Open questions concerned special progress on a particular day and whether subjects experienced a definite moment of success.

Since subjects with tetraplegia were familiar with wheelchair control, they additionally evaluated the ACS on the same answering scale (e.g. fitness for daily use, personal preference, several driving maneuvers). Finally, they completed the NASA TLX (perceived workload: mental, physical and temporal demand, performance, effort and frustration (Hart \& Staveland, 1988; Rubio, Diaz, Martin, \& Puente, 2004)) with a visual rating scale ranging from 0 (very little) to 100 (very high).

\subsubsection{Statistical Analysis}

Results from healthy subjects for each performance criterion was analyzed using SPSS (IBM, Version 19, IBM, Armonk, U.S.). Data from left and right ear was pooled after nonsignificant Repeated Measures ANOVAs (all $p>0.05$ ).

Training effects were analyzed with repeated measures ANOVAs with the factor "time". Performance differences between session 1 and session $5 \mathrm{a}$ and $5 \mathrm{~b}$ were further analyzed with t-tests for paired samples or the Wilcoxon Rank test, if the criterion of normal distribution was not met (Shapiro-Wilk test). All p-values were compared to a Bonferroni-corrected alpha-level of $0.025\left(\alpha_{\text {corr }}=0.05 / 2=\alpha_{\text {global }} /\right.$ number of tests $)$ to minimize the risk of false positive results (Bland \& Altman, 1995). T-tests were used for differences between EW and NEW (independent samples, sessions 1, 5a and 5b, $\alpha_{\text {corr }}=0.05 / 3=0.016$ ) and the questionnaire about PAM control (paired samples, Mean session 1: M1, mean session 5: M5). 


\subsection{Results}

\subsubsection{Training effects on ACS performance}

For the able-bodied subjects $(n=10)$, repeated measures ANOVAs revealed training effects for all eight performance criteria (all $p<0.05$, Supp. Table 1). The effect size Etasquared ranged between 0.249 (reaction time for contraction) and 0.545 (repetition rate of contractions), with a mean of 0.405 (Supp. Table 1).

In general, the performance had improved significantly by session $5 \mathrm{a}$ and continued to improve during the last session of wheelchair driving (session 5b, see Figure 10 and Supp. Table 1) for results of dependent t-tests, $\alpha$ corr=0.025). More concretely, the mean reaction times for contractions decreased from $585 \mathrm{~ms}$ in the first session to $408 \mathrm{~ms}$ in session 5b (Figure $10 \mathrm{~A}$ ) as did reaction times for relaxation (692 ms to $462 \mathrm{~ms}$, Figure 10 B). Subjects thus learned to activate and relax their PAM faster. The maximum contraction rate reflected this, it increased significantly from an initial $1.057 \mathrm{~Hz}$ to a maximum $1.408 \mathrm{~Hz}$ in session 5a (Figure $10 \mathrm{C}$ ). In lateralized activation, the percentage of correct activations doubled from $26.7 \%$ in session 1 to $50.6 \%$ in session $5 \mathrm{~b}$ (Figure 10 F). This means control over the ACS improved with training, as confirmed in the virtual obstacle course (Figure 11). The length of the driven path (Figure $10 \mathrm{G}$ ) decreased significantly from 4036 pixel in session 1 to 2997 pixel in session 5b, as did completion time (Figure $10 \mathrm{H}$ ), which decreased by more than half, from $127.4 \mathrm{~s}$ to $62.5 \mathrm{~s}$.

Performance in graded PAM activation and maximum contraction duration were exceptions. They benefitted especially from wheelchair driving in the last session $5 \mathrm{~b}$, but did not attain statistical significance in session 5a. Graded PAM activation improved from $63.4 \%$ in session 1 to $75.1 \%$ in session 5 a, with an additional increase to $80.6 \%$ in session $5 b$ (Figure 10 D). Similarly, in maximum contraction duration subjects held contractions for $15 \mathrm{~s}$ longer before wheelchair driving than initially (session 1: $50.3 \mathrm{~s}$, session 5a: $64.8 \mathrm{~s}$, Figure $10 \mathrm{E}$ ) with an extra $5 \mathrm{~s}$ after the wheelchair session (session 5b: $70.6 \mathrm{~s})$.

Finally, we looked for differences between the five initial "ear-wigglers" (EW) and the five initial "non-ear-wigglers" (NEW, see Supp. Fig. 7 for examples) in the able-bodied volunteers. Prior ability to wiggle the ears seems to have an impact on lateralized activation ability. Although both groups improved their performance by almost $100 \%$ 
from the first session to the last, EWs still had a higher final score than NEWs (session 1: $M_{E W}=32 \%, M_{N E W}=21.3 \%, t(8)=0.914, p=0.388$; session $5 \mathrm{a}: M_{E W}=62.7 \%, M_{N E W}=38.4 \%$, $t(8)=2.732, p=0.026$, Supp. Fig. 7 C). In most performance criteria however, no differences were found between the two groups. In summary, the performance of the ACS improved throughout the course of training in all able-bodied participants. The training effect seemed to be mostly independent of the initial ability to wiggle the ears except for lateralized activation. 

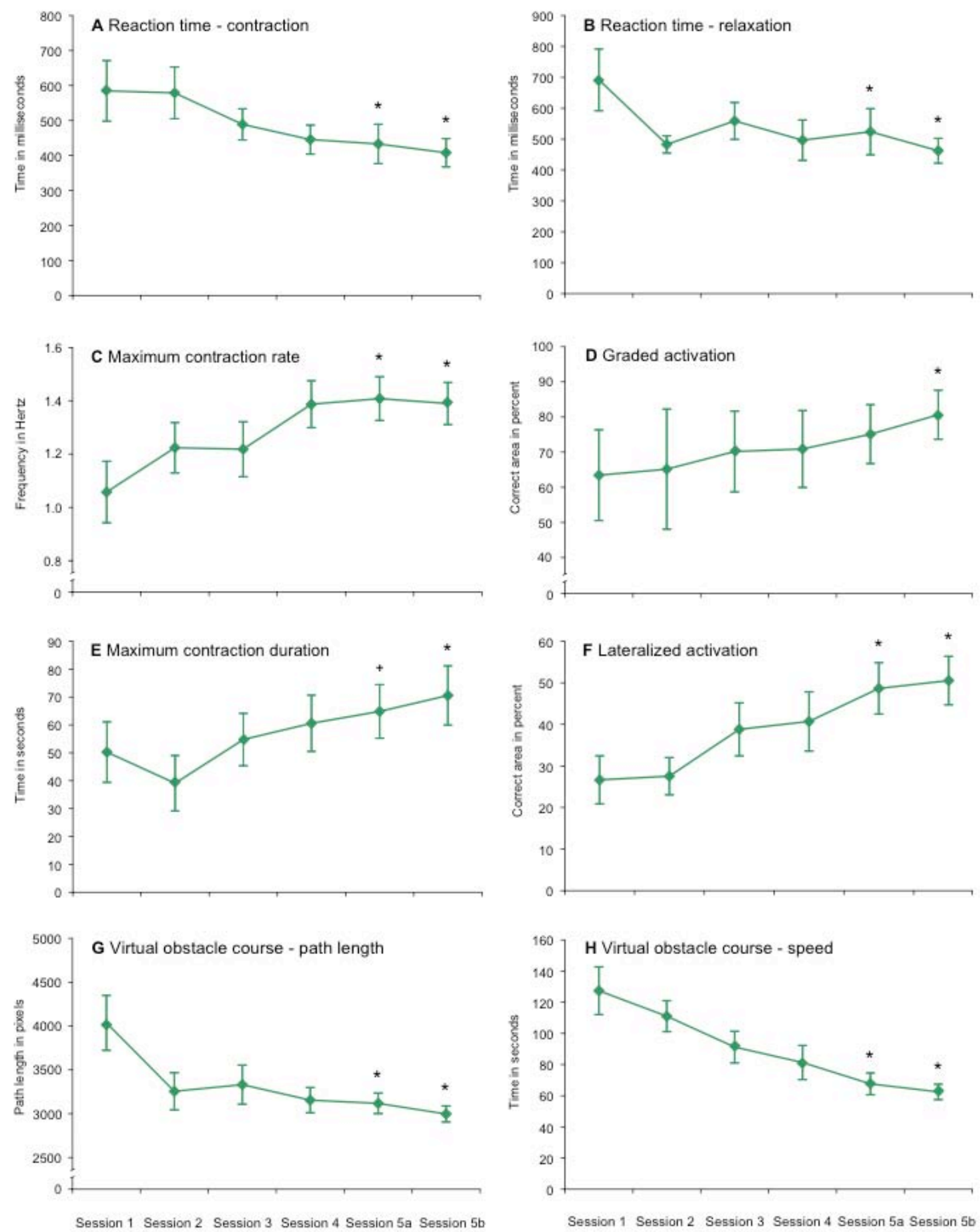

\section{Figure 10) Positive training effects on PAM activation.}

Mean results with standard error of ten able-bodied subjects in eight tests of the PAMs' activation performance during four days of training (session 1 - session 4) and before and after powered wheelchair driving (session 5a, session 5b). Subjects were tested for reaction time of PAM contraction (A) and relaxation (B), contraction rate (C), graded activation (D), maximum contraction duration (E) and lateralized activation (F). In addition, driving skills on a virtual obstacle course were tested as path length $(\mathrm{G})$ and speed $(\mathrm{H})$. Significant differences between session 1 and session $5 \mathrm{a}$ or session 1 and session $5 \mathrm{~b}$ as tested with paired $t$-tests or Wilcoxon Rank test are marked $+=p<0.05$ or ${ }^{*}=p<0.025$ (Bonferroni corrected alpha level). 


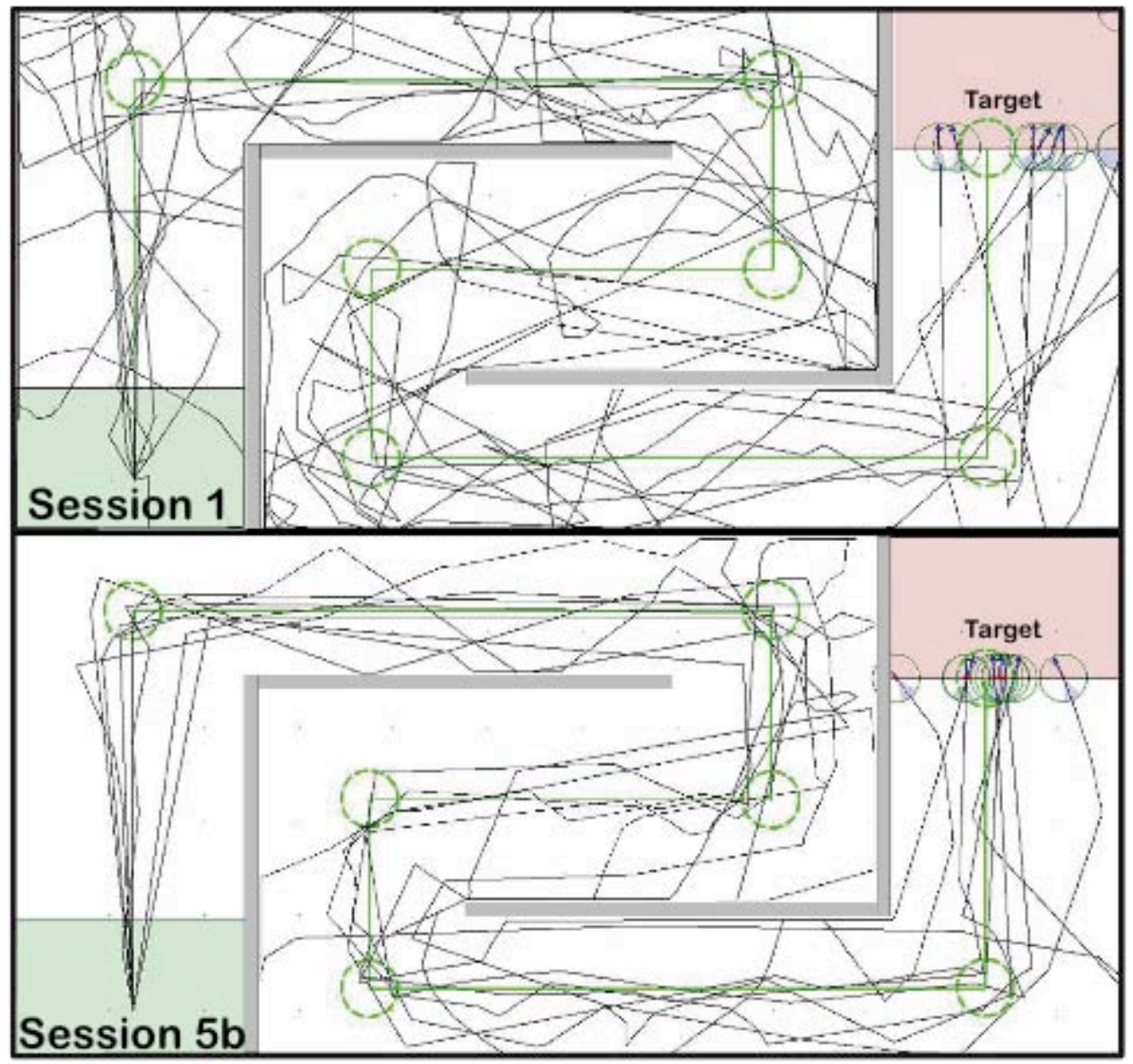

Figure 11) Improved performance using the ACS in a virtual obstacle course.

Individual paths (back lines) of able-bodied subjects in session 1 using the ACS. Training resulted in a decreased number of turns, collisions, and the path length in session $5 \mathrm{~b}$.

Training effects of the participants with tetraplegia were similar to able-bodied subjects (Figure 12 for examples). In general, T2 performed slightly below the level of the ablebodied subjects, while T1 performed slightly better. In most tests, their performance was still within the range of one standard deviation of the able-bodied subjects' performances. Due to general physical and mental exhaustion after wheelchair driving, session $5 \mathrm{~b}$ was skipped for subject $\mathrm{T} 2$, as well as the contraction duration measurement in session $5 \mathrm{~b}$ for subject $\mathrm{T} 1$.

Regarding reaction times, T1 was able to further improve his already high performance in the beginning in both contraction (session 1: $346 \mathrm{~ms}$, session 5b: $272 \mathrm{~ms}$, see Figure $12 \mathrm{~A}$ ) and relaxation times (session 1: $561 \mathrm{~ms}$, session 5b: $73 \mathrm{~ms}$ ). T2's reaction times varied strongly. For example, although T2 showed notable improvements in session 4, 
his PAM contractions were more delayed in session 5 a than in the beginning (session 1: $1008 \mathrm{~ms}$, session 5b: $1508 \mathrm{~ms}$, see Figure $12 \mathrm{~A}$ ). The same was true for relaxation time (session 1: $979 \mathrm{~ms}$, session 5b: $1359 \mathrm{~ms}$ ). Nevertheless, both subjects with tetraplegia improved their rate of maximum contractions notably. T1 managed to improve his initial $1.22 \mathrm{~Hz}$ to $1.55 \mathrm{~Hz}$ in session $5 \mathrm{~b}$, while T2 also improved, although to a lower level (session 1: $0.65 \mathrm{~Hz}$, session 5a: $0.93 \mathrm{~Hz}$ ). Both subjects did not improve their fine muscle control as measured by graded PAM activation (T2 session 1: 79.12\%, session 5a: 79.75\%, see Figure 12 B). T1's precision in PAM activation even decreased (session 1: 65.58\%, session 5b: 61.72\%). Here, a ceiling effect appeared, since both subjects performed above the mean of the able-bodied subjects (Figure $12 \mathrm{~B}$ ).

The maximum time of contraction again showed variations. Although T1 in the end was able to generate a slightly longer persistent PAM contraction (session 1: $43.9 \mathrm{~s}$, session 5a: $58.6 \mathrm{~s}$ ), this individual had some performance drops (e.g. session 3: $22.1 \mathrm{~s}$ ). The same applies for T2 (session 1: $34.2 \mathrm{~s}$, session 3: $7.3 \mathrm{~s}$, session 5a: $45.8 \mathrm{~s}$ ). The ability to lateralize PAM activation, on the other hand, improved notably in both subjects (Figure $12 \mathrm{C})$.

The driving skills measured in the virtual obstacle course improved notably in both subjects (Figure $12 \mathrm{D}$ and E, Figure $13 \mathrm{~B}$ ). T2 needed $30 \mathrm{~s}$ less at the end compared to onset of training to complete the course (session 1: $139.5 \mathrm{~s}$, session 5a: $104.9 \mathrm{~s}$ ). T1 improved by $20 \mathrm{~s}$, although his initial performance was already high (session 1: $91.0 \mathrm{~s}$, session 5b: $71.6 \mathrm{~s}$ ), with a maximum speed of only $50.3 \mathrm{~s}$ on session 4 . Both subjects shortened the driving path from 3993 pixels to 3409 pixels (T2) and from 4853 pixels to 2680 pixels, respectively (T1). 

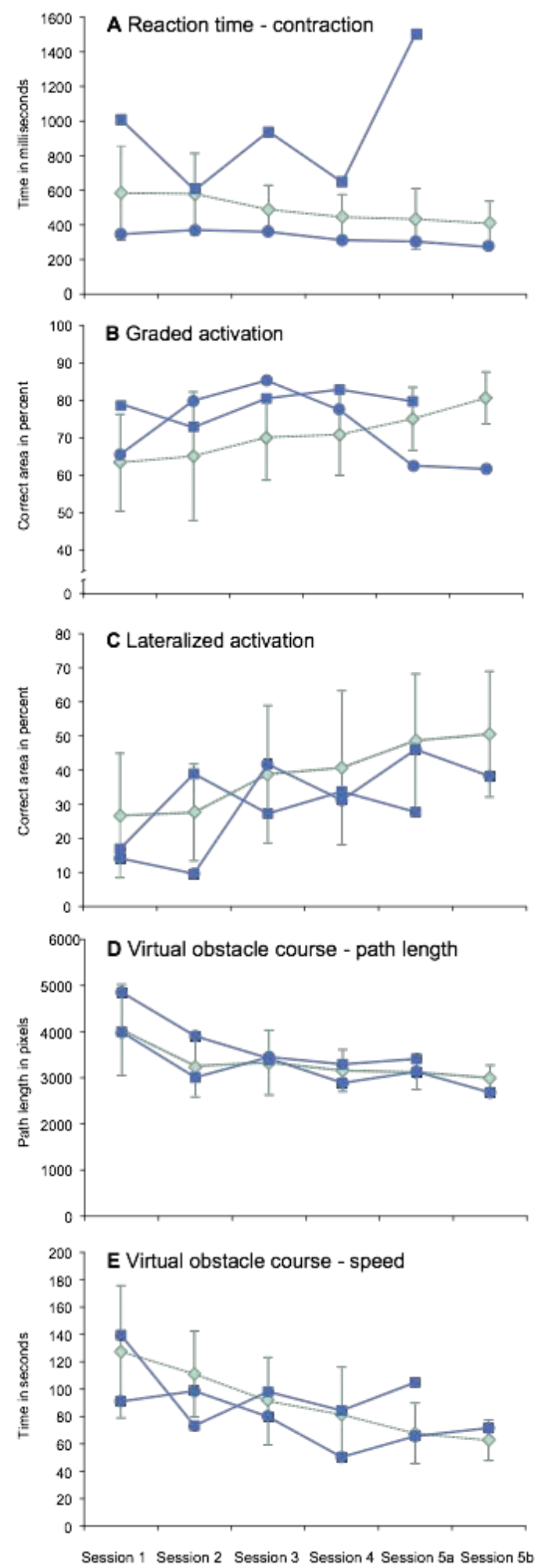

\section{Figure 12) Training effects of subjects with tetraplegia.}

Individual results of T1 (blue lines, circles) and T2 (squares) compared to able-bodied subjects' mean results (green line) with standard deviations in selected PAM performance criteria: reaction time of contraction (A), graded activation (B), lateralized activation (C) and path length (D) and speed (E) in a virtual obstacle course. 


\subsubsection{Wheelchair driving with PAM EMG-generated control signals}

In the second part of the study (session $5 \mathrm{~b}$ ), all subjects controlled a wheelchair with the ACS. All subjects, including those with tetraplegia, were able to drive the wheelchair along a straight, three-meter wide corridor for a distance of at least four meters by full co-activation of the PAMs on both sides. All managed to turn on command up to $360^{\circ}$ in both directions by selectively activating one side. In addition, both subjects with tetraplegia successfully completed a complex obstacle course (Figure 13 A). Both had one collision, but managed to complete the course within a reasonable time (T1: $86 \mathrm{~s}$; T2: $201.6 \mathrm{~s})$.

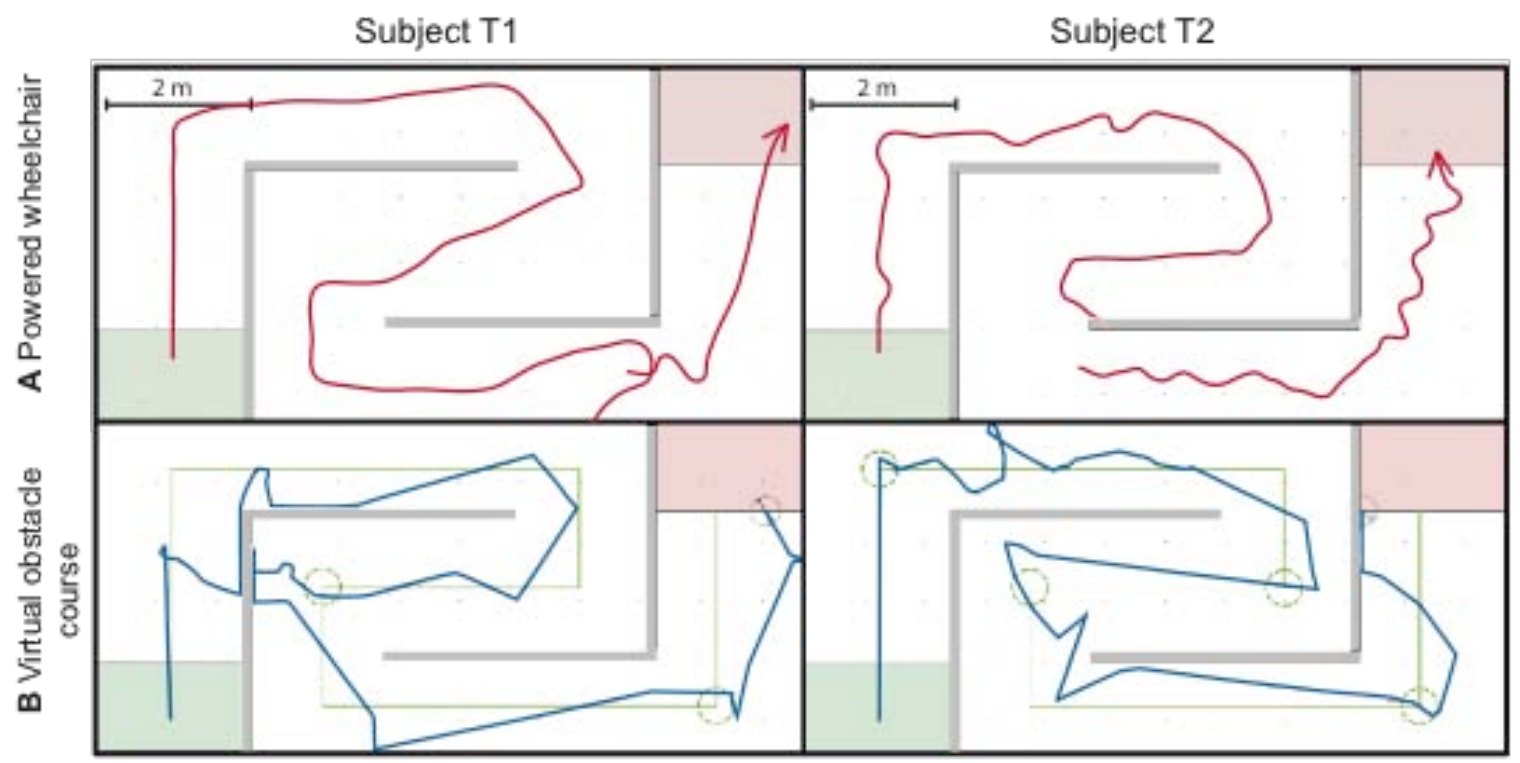

Figure 13) Real and virtual wheelchair driving using the ACS.

Powered wheelchair driving paths (A) of two subjects with tetraplegia in a real obstacle course. The path was filmed and manually digitalized into the corresponding virtual obstacle course. Subject T1 needed 1.45 min and subject T2 3.36 min.; both had one collision. Blue lines (B) show driving paths in an analogue virtual obstacle course in test 5 a (before wheelchair driving).

\subsubsection{Subject-reported outcomes on training and ACS performance}

Participants evaluated their ability to control PAM activation (four-point answering scale, $1=$ not at all, 4= a lot) to have improved during the study from a "moderate" level $\left(M_{1}=2.7\right)$ in the first training session to an almost maximum level $\left(M_{5}=3.8, t(9)=-6.128\right.$, $p=0.000$ ) at the end. When divided into subgroups, ear-wigglers initially rated their ability for PAM activation higher than NEW $\left(M_{E W, 1}=3.2, M_{N E W, 1}=2.2\right)$, but NEW almost 
reached the top level in the end $\left(M_{E W, 5}=4.0, M_{N E W, 5}=3.6\right)$. Subjects generally considered that their ability to activate each PAM separately had improved substantially with training (right PAM: $M_{1}$ 1.7, $M_{2} 3.25, t(9)=-6.012, p=0.000$; left PAM: $M_{1} 1.6, M_{2} 3.35$, $t(9)=4.389, p=0.002)$. These results actually reflect the objective results from the performance criteria. The required mental effort and concentration during the procedure decreased, though not significantly, as did self-perceived co-activation of facial muscles.

Subjects with tetraplegia considered the ACS to be technically mature enough for everyday use $(M=3.7)$ and were eager to use it $(M=4)$. Difficulty in precise command generation was judged to be "small" $(M=2.25)$. Specific driving maneuvers such as narrow or wide turns, and precision and time to reaction received medium scores $(M=2.5$ - 2.75). Both used a joystick control in daily life and hence called hands-free control a major advantage of the ACS. Suggestions for improvements were a selfcontrolled emergency stop and a reverse gear as well as control over electronic devices as an additional application area for the ACS. For safely using the ACS, T2 expected seven and T1 14 more necessary training days.

The NASA TLX (Task Load Index, answering scale: 0=very low - 100=very high) revealed a low general workload for subject T1 $(M=27)$ and medium workload for T2 $(M=57)$ when using the ACS. T1 gave medium scores to the subscales of mental demand and effort (50 each), while he judged the physical demand to be low (30). Surprisingly, he also judged his success as low (30). Temporal demands and frustration on the other hand did not matter to T1 at all (0). Frustration was also not important for T2 (0), however, mental, physical and temporal demand received medium-high scores (70). T2 had to put in a medium effort (50), and judged his own performance to be medium (50). 


\subsection{Discussion}

This study revealed two novel findings. First, voluntary activation of the auricular muscles, the prerequisite for an ACS, can be learned and trained in a short period of time. All subjects improved in various tests of the ACS's performance, including the important lateralization of PAM activation. The second major finding is that the ACS can serve as an alternative control system. Precise signal generation made it possible to control a powered wheelchair at a high performance level. Subjective evaluation was highly positive, rating the ACS as ready for daily use.

\subsubsection{Voluntary control of PAM activation}

The limited research on the auricular muscles reports conflicting evidence on whether humans can intentionally activate the auricular muscles (Berzin \& Fortinguerra, 1993; Serra et al., 1986). Our results, however, clearly demonstrate that the auricular muscles can be voluntarily controlled and that this control can be learned and trained. The training effect was most impressive in those subjects who had declared themselves unable to control their PAMs in the beginning. These non-ear-wigglers were actually able to attain the performance levels of the ear-wigglers in most of the studied outcome measures. These results indicate that control over the PAMs is largely independent of previous ability, is easy to learn and that considerable performance can be achieved even with little training. One exception is lateralized activation. Longer training may help the NEW to equal the EW's proficiency in this task, but this has to be shown in future studies. Participants with tetraplegia showed more unstable results than ablebodied subjects, however, since the group size was small we do not want to draw conclusions from this. Possible reasons for a difference might be their use of spasmolytic medication and participation in the regular intensive clinical therapy program (around six hours of daily physical and mental exercises).

The voluntary control of the PAMs learned by our subjects is not limited to a basic activation. Participants learned to perform complex actions, such as control over a lateralized and graded PAM activation or the rate of sequential contractions. Interestingly, the learning curve for this new skill is not necessarily a uniformly continuous increase. Sporadic or selective improvements (see e.g. Figure 10 C, F and B) 
can be partly explained by reported moments of insight, in which subjects "suddenly" understood what they had to do in order to activate their PAMs. This moment reportedly occurred most often on day three. This is directly reflected in the performance improvements on day three in lateralized activation and in the reaction time for contraction, but also in maximum contraction duration (Figure $10 \mathrm{~F}, \mathrm{~A}$ and $\mathrm{E}$, respectively). These results prove that control over the PAMs is easy to learn and considerable performance can be achieved even with a short training period.

The visual feedback of EMG activity used in our study was reported to be especially helpful for skill acquisition, as has been observed in training of voluntary activation of other inaccessible muscles (e.g. pelvic floor muscle or sphincter muscles (Theofrastous et al., 2002)). Results suggest that the immediate senso-motor feedback when controlling a wheelchair with the PAM might be even more effective: a contraction of the right PAM, for example, moves the whole body into the desired direction. Accordingly, subjects with tetraplegia had fewer collisions in a real world obstacle course than in the virtual obstacle course (Figure 11). The intuitive control commands of the ACS might thus increase the learning effect further with longer wheelchair sessions.

The ability to voluntarily control the auricular muscles suggests an involvement of the primary motor cortex (M1). Training control over the auricular muscles may thus have a direct influence on the neuronal plasticity of the involved cortical area. A neuronal reorganization caused by training usually interacts with an enhanced performance, e.g. in complex motor tasks (Karni et al., 1995). It is therefore likely that longer training of voluntary PAM activation would lead to a neuronal reorganization that would then further increase control over PAM activation. Since the motor cortex is widely inactive in subjects with tetraplegia, one might speculate that they have a higher training potential than able-bodied impaired individuals. Future studies should investigate whether longer training periods indeed lead to a neuronal reorganization and whether this would perhaps enable a more differentiated control over the muscles, for example separate activation of the posterior and superior auricular muscles. 


\subsubsection{Application of the ACS for wheelchair control}

Regarding the second finding, the results demonstrate that auricular muscle-generated EMG signals can serve as precise command signals. Improvements in driving capabilities resulted in higher speed and shorter driven path length in virtual wheelchair driving and in the successful generation of basic steering commands in a powered wheelchair.

Similar to other reports on wheelchair control systems (Han, Zenn Bien, Kim, Lee, \& Kim, 2003; J. D. R. Millán et al., 2009), more advanced wheelchair driving capabilities were assessed in the two subjects with tetraplegia since they are the main target group for this type of technology. Although these participants had no experience in ACS wheelchair control, they managed complex driving situations that approximate those of real life with only one collision each. In comparison to other control systems, a trapezius muscle controlled system produced poorer results with four to seven collisions on a straight 10 m track (Han et al., 2003). Brain Computer Interfaces (BCI) suffer from a low information transfer rate, low robustness and a high likelihood of false commands (Ryu \& Shenoy, 2009; Wolpaw et al., 2002), which makes precise wheelchair driving challenging (e.g. subjects completely failed to reach a designated position even with intelligent wheelchairs (J. D. R. Millán et al., 2009) or had difficulties reaching a target 2 $\mathrm{m}$ away (Tanaka et al., 2005)). The performance of subject T2 furthermore indicates that even with below-average results on the performance criteria, complex control signals can be successfully generated with the PAMs. Most notably, despite the short adjustment period (five minutes) subjects with tetraplegia reported only low-to-medium workload on the NASA TLX for using the wheelchair control system.

Precise signal generation determines safety aspects in electric wheelchair driving. With the ACS, users must avoid involuntary activations that can lead to false commands. Participants with tetraplegia estimated that they would require only 7 to 14 more days of training to achieve complete and safe control of the wheelchair using the ACS.

Besides precision, user safety largely depends on the ability to quickly and flexibly modulate the control signal, e.g. in the event of a sudden dangerous situation. Slow control signal modulation is a major drawback of some control systems, such as BCI (Diez et al., 2013; J. D. Millán et al., 2010), but also of voice control (Simpson \& Levine, 2002; Youdin et al., 1980). In order to avoid collisions when driving a wheelchair, shared control based on external sensors or intelligent algorithms (Iturrate, Antelis, \& Minguez, 
2009; J. D. R. Millán et al., 2009) can be employed, but this comes at the cost of individual control and flexibility. In our study, this signal speed was tested with reaction times for PAM contractions, which decreased with training to an average of $408 \mathrm{~ms}$. This is surprisingly close to reaction times for the right index finger that ranges from $280 \mathrm{~ms}$ to $320 \mathrm{~ms}$ for visual cues (Niemi \& Naatanen, 1981). A more detailed analysis of our data revealed that six individuals managed to generate an above-threshold PAM activation speed of even less than $400 \mathrm{~ms}$, with a mean reaction time of $323 \mathrm{~ms}$ (SD $39 \mathrm{~ms}$ ). Interestingly enough, half of these were initial non-ear-wigglers. These fast reaction times after such a short training time demonstrates the potential that the PAM has in signal generation. Fast signal generation results not only in better safety, but also permits higher driving speed, which considerably increases users' satisfaction with driving.

Another safety issue is the so-called Midas Touch problem. For control systems it is a challenge to distinguish between intentional and an unintentional signal, e.g. a signal gaze and a normal gaze in eye-tracking control (Barea et al., 2002). The advantage of the ACS becomes clear especially in this context: since the auricular muscles assume a function only in the context of the ACS, there is no interference with an original function as in the case of the eyes or hands. The ACS is hence minimizing the risk of involuntary commands.

Compared to other control systems, signal generation with the ACS has two special characteristics. First, the control is proportional: signal strength depends on muscle contraction strength. Results from e.g. graded PAM activation and online modification of the real wheelchair driving course suggest that the user can modulate the amplitude of the control signal by using different degrees of PAM activation in an analogue manner. Second, EMG signal transmission is continuous. The control signal is thus generated for as long as the muscle contracts, allowing constant control over the output action. Continuous signals are a major advantage over simple absolute signals, where subjects can only decide e.g. between $90^{\circ}$ turns or driving straight ahead, without the option to modify the command in real time, as it is the case e.g. with the basic sip-and-puff control system (Kim et al., 2013). Generating continuous command signals also remains a major challenge in BCI research (Diez et al., 2013; Donoghue, 2008; Wolpaw et al., 2002). 
The combination of both features in the ACS supports a joystick-like performance. They open a wide field of application for the ACS, particularly whenever a precise, constant control over an output action is required. When used to steer a wheelchair, users can simultaneously control the strength of the translational and rotational wheelchair movements, e.g. steering forward and left at the same time using bilateral PAM contractions with more PAM activation on the left side. In contrast, other control systems require the wheelchair to completely stop before rotational steering movements can be initiated (Simpson \& Levine, 2002; Tanaka et al., 2005). The possibility to drive S-curves on a slalom course with our system was hence particularly valued by our subjects with tetraplegia.

Additional commands can be assigned conveniently in the frame of a serial command, e.g. two short co-contractions switch the system to backgear. With the ACs, we have three raw signals (left ear, right ear and co-contraction) that can be combined to carry out additional commands.

We have demonstrated here that the PAM, hitherto regarded as rudimentary muscles with no dedicated function, can take on a complex control function. This differs from other control systems, e.g. tongue control (Kim et al., 2013; Lontis et al., 2010; Struijk, 2006) or even simple hand control, in which already present motor functions are assigned an additional purpose and thus interfere with the original motor activity. The sole control-purpose of this muscle minimizes the risk of unintentional commands, also known as the Midas Touch Problem. With the integration of the PAM into a new functional context, the ACS makes full use of the unexploited potential that this muscle has, particularly for individuals with tetraplegia, in whom only a few motor functions are preserved.

\subsubsection{Evaluation of the ACS: social implications}

Evaluation of the subjects with tetraplegia focused on an often-overlooked disadvantage of established wheelchair control systems, namely that they interfere with social interaction. Unlike the chin control or any mouth based control (Kim et al., 2013), activation of the PAM allows subjects to simultaneously move their head, chew and even talk while controlling the wheelchair, which confirms an earlier case report where PAM 
activation and speech did not interfere (Foldes \& Taylor, 2010). Only broad smiling provoked a PAM co-contraction in some cases. Thanks to the isolated position of the PAM, the social advantage over already existing systems therefore remains considerable. Independence is another crucial topic for individuals with tetraplegia. In tetraplegics who have a sense of enablement, depression scores do not differ from the general population (Middleton et al., 2007). Aside from greater independence in wheelchair control, an integrated domestics control system using the ACS could easily improve the self-sufficiency and thus life quality of persons with severe motor impairments by providing e.g. access to electronic devices or social media, as suggested by our subjects with tetraplegia. The ACS thus has great potential not only to improve mobility for persons depending on electric wheelchairs, but could also have a positive influence on social and psychological aspects, thereby enhancing their quality of life.

\subsection{Conclusion}

We showed that voluntary control of the auricular muscles can be learned by training. Motor learning in auricular muscle control does not proceed continuously but is rather determined by moments of insight. It is largely independent of prior proficiency and includes control over complex activation patterns, thus fulfilling the prerequisites for an auricular-based control system. This proof-of-principle should now be replicated with bigger sample sizes. The results of the powered wheelchair driving tests demonstrated that the ACS is an intriguing alternative to existing control interfaces. Its characteristics, such as short training time, proportional and continuous control as well as high signal quality and speed, and intuitive signal generation render it attractive not only for subjects with tetraplegia, but also for other wheelchair users and even application beyond the clinical use, whenever hands-free control is advantageous. 


\section{Independent control of two degrees of freedom in prosthetic hands with the ACS}

After providing evidence of the suitability of the ACS for precise signal generation in wheelchair control, we aimed to test its application in another important field of assistive devices, namely prosthesis control.

In this study, we evaluated whether integrating the ACS into an established prosthesis control scheme (hybrid ACS, hACS) can solve the problem with prosthesis control outlined in the introduction. Clinically established control modes can only control one active DOF at a time (Muzumdar, 2004; Zecca et al., 2002) which makes the use of a prosthetic hands slow and results in low acceptance rates (Wright et al., 1995). Likewise, these control interfaces cannot answer to the complexity of newly developed hand prostheses with several degrees of freedom (Atzori \& Muller, 2015; Belter et al., 2013; Farina et al., 2014).

Other alternatives proposed by the research community have not made a clinical impact due to technical issues (pattern recognition (Ning Jiang et al., 2012)) or interference with daily activities (e.g. foot switch, throat microphone (Carrozza et al., 2007; Mainardi \& Davalli, 2007). For the clinically established control options, typically the electrical activity of an agonist/antagonist muscle pair in the forearm is measured with surface electrodes and used to control either rotation or opening/closing of the prosthetic hand. In the following study, the same set up is used while additional muscle signals from the bilateral posterior auricular muscles allow the control over a second DOF. This hybrid ACS can provide an independent, simultaneous, proportional control of two DOFs of prosthetic hands. Ten able-bodied subjects and one subject with transradial amputation tested the performance of the hACS and the clinically established slope control (SL) and co-contraction (CC). Although subjects were untrained in all three controls, we expected them to perform better in two established tests using the hACS due to its easier control logic.

This study is prepared for publication under the title: "A hybrid auricular control system: direct, simultaneous, and proportional myoelectric control of two degrees of freedom in prosthetic hands." (Schmalfuss et al., n.d.). 


\subsection{Methods}

\subsubsection{Study protocol}

Ten able-bodied subjects (mean age: $28.5 \pm 7.8$ yrs; 3 female, 7 male) and one 50-yearold man with a transradial amputation participated in the study. In order to keep training time to a minimum we included subjects who were able to activate their ear muscles voluntarily. The research protocol was approved by the ethics committee of the University of Göttingen and was in accordance with the latest version of the Declaration of Helsinki. Written informed consent was obtained from each participant.

We compared two well-established prosthesis control interfaces, CC and SL, with the hACS. The subjects were allowed ten minutes to accustom themselves with each control interface before testing began. Each subject used the three interfaces to control the prosthetic hand (co-contraction, slope control, auricular control, each for approx. one hour). A session lasted approximately four hours.

Performance was compared using two validated tasks with practical relevance. In the clothespin relocation test (Amsuess, Goebel, Graimann, \& Farina, 2014; Hussaini \& Kyberd, 2016), subjects transferred three pins of the Rolyan Graded Pinch Exerciser ( $\sim 10 \mathrm{~N}$ grip force) from a horizontal to a vertical bar. The completion time (speed) and number of dropped pins (errors) were recorded. In the box and blocks test (Mathiowetz, Volland, Kashman, \& Weber, 1985) the number of square blocks that subjects were able to move from one box to another in one minute was counted. In this simple grasprelease task, rotation causes delays and results in fewer transported blocks. This test is, therefore, sensitive to false-positives in rotational control.

Since the position of the arm can affect accurate prosthesis control (Fougner et al., 2011; Ning Jiang et al., 2013), the clothespin test was conducted in three arm positions (low = test is located at knee height, middle = test is located at hip height, high = test is located at shoulder height). Five repetitions per arm position were performed in the same order for all subjects (middle, high, low), and each arm position was tested twice with each control interface (block 1 and block 2, 30 items total per control interface). To eliminate the potential bias of cross-method learning, the order of the three interfaces was randomized across subjects. 


\subsubsection{Prosthesis and control interfaces}

The artificial hand (System Electric Hand DMC plus with rotation unit, Otto Bock, Duderstadt, Germany) was affixed to a custom-made cast arm shaft, with a tube for the electronics, and the subject's arm was fixated in the shaft (Figure 14 a).

For all three control interfaces, bipolar surface EMG-amplifiers (13E200, Otto Bock, Duderstadt, Germany) were placed over the flexor and extensor muscles of the wrist (Figure 14 a). CC and SL were investigated with a commercially available control unit (Otto Bock, "Myorotonic"), while the hybrid ACS was implemented with a custom-made interface and control logic.

A)

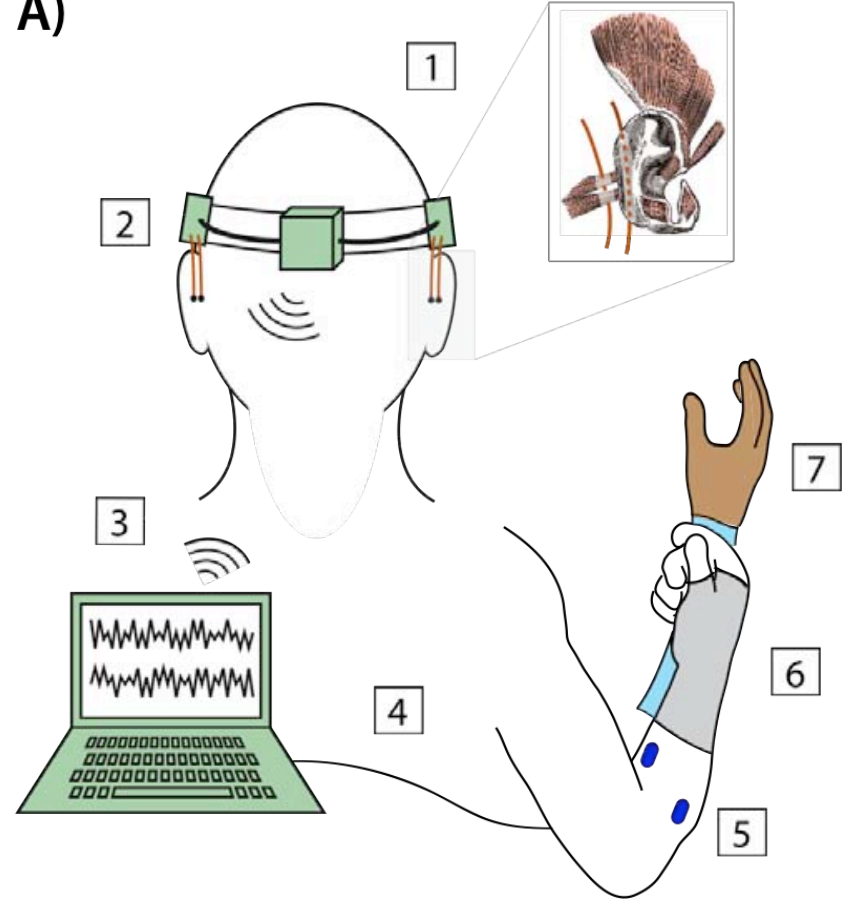

B)

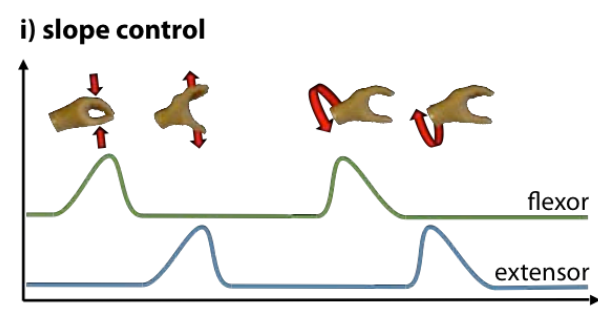

ii) co-contraction control

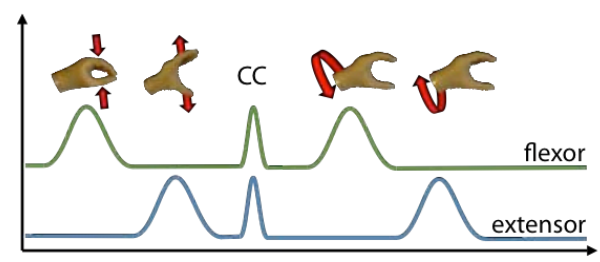

iii) hybrid auricular control

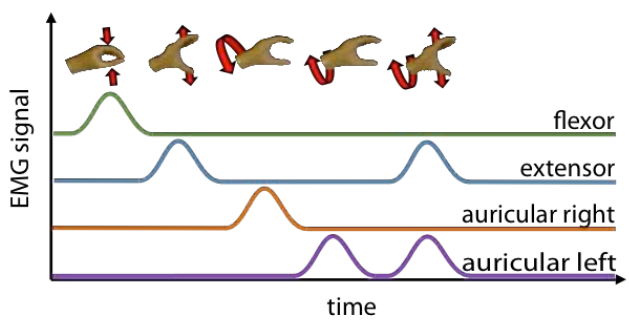

Figure 14) Set-up of the auricular control system (A) and signal generation for the control interfaces (B).

In the ACS (A), fine wire electrodes were inserted subcutaneously over the posterior auricular muscle and behind the pinna (1). The ends of the electrodes were connected to amplifiers mounted on a headset worn by the subject (2). The EMG signals were transmitted by a wireless Zig-Bee connection to the computer (3), where a specially developed software converted them into rotational signals that were transmitted by cable (4) to the prosthetic hand. For all three control systems, Otto Bock ${ }^{\mathrm{TM}}$ surface electrodes were fixed on the flexor and extensor muscles of the subjects' arm (5). The prosthetic hand was affixed to an arm shaft (6) that was put over the subjects' healthy hand. With the combination of the auricular and forearm signals, the prosthetic hand (7) could be rotated and opened /closed simultaneously. For signal generation (B) with slope (SL) control (i), a slow contraction of the flexor muscles closed the prosthesis, while a slow contraction of the extensor muscles opened it. A fast contraction of the flexors rotated the 
hand to the right and a fast contraction of the extensors rotated it to the left. A small pause was obligatory between the different action modes. With co-contraction (CC) control (ii), contracting the flexors closed the hand and contracting the extensors opened it. In order to rotate the hand, a simultaneous contraction of flexors and extensors (co-contraction) was required. Then, the contraction of the flexor produced a clockwise rotation of the prosthetic hand and a contraction of the extensor a counter clockwise rotation. With the auricular control (iii), activating the flexors and extensors closed and opened the hand, respectively. Activating the right auricular muscle rotated the prosthesis clockwise, while activating the left auricular muscle rotated it counter clockwise. A simultaneous activation of, e.g., the extensors and the left auricular muscle opened the hand and rotated it counter clockwise at the same time.

In $\mathrm{CC}$, activation of the flexor muscles closed the hand while activation of the extensor muscles opened it (Figure $14 \mathrm{~b}, \mathrm{i}$ ). In order to switch the actuation DOF of the prosthetic hand, the subjects had to generate a co-contraction of both muscle groups. After changing the actuation mode, activating the flexor muscles pronated and the extensor muscles supinated the prosthetic hand. A further co-contraction re-established control of opening and closing, giving a sequential control of the two actuation DOFs.

A similar principle applied for slope control (Figure $14 \mathrm{~b}$, ii). Here, the speed of a single contraction was used to determine the task. A slow contraction of the extensor muscles opened the hand whereas a quick contraction of the same muscles supinated the hand. The flexor muscles determined hand closing and pronation in the same manner.

For the hACS (Figure $14 \mathrm{~b}$, iii), activating the extensor and flexor muscles opened and closed the hand, respectively. Wrist rotation was proportionally controlled by the auricular muscles (left rotation by left auricular muscle, right rotation by right auricular muscle). With this approach, both actuation DOFs could be controlled simultaneously in a proportional manner and the actuation speed could be varied independently. Calibration and set up of the hACS was done as described in chapters 2.1.1 and 2.1.2. The difference between the normalized left and right PAM signals was converted via a custom-made interface into a pulse-width modulated signal to directly drive the prosthesis motor of the rotation unit proportionally in both directions.

\subsubsection{Statistical analysis}

For the clothespin relocation test, every subject performed 30 runs with each control interface, resulting in a total of 300 items per control interface for all subjects. We calculated two different repeated measures ANOVAs for the clothespin relocation data set (control interface $\mathrm{x}$ arm position; control interface $\mathrm{x}$ time) and analyzed the effects 
with post-hoc two-tailed t-tests. For each analysis the alpha level was Bonferronicorrected for multiple testing (type of control interface: $\alpha_{\text {corr }}=0.5 / 3=.0167$, arm position: $\alpha_{\text {corr }}=0.5 / 3=.0167$, time: $\alpha_{\text {corr }}=0.5 / 3=.0167$ ). For the first ANOVA, Mauchly's test indicated that the assumption of sphericity was violated for the main effect of control interface, $\chi^{2}(2)=20.16, p=.00$, of arm position $\chi^{2}(2)=8.65, p=.013$ and of the interaction $\chi^{2}(9)=29.57, p=.001$, therefore degrees of freedom were corrected using Huynh-Feldt estimates of sphericity ( $\varepsilon=.85$ for main effect of Control, $\varepsilon=.94$ for main effect of arm position and $\varepsilon=.90$ for interaction).

The data set of the box and blocks test was analyzed with another repeated measures ANOVA. Again, sphericity was violated, $\chi^{2}(2)=10.08, p=.006$, and Huynh-Feldt estimates of sphericity were used $(\varepsilon=.85)$. Three post-hoc t-tests were calculated and the alpha level was Bonferroni-corrected ( $\left.\alpha_{\text {corr }}=0.5 / 3=.0167\right)$.

The data of the subject with the transradial amputation was not used in the statistical analysis; only descriptive statistics are provided for these data.

\subsection{Results}

\subsubsection{Functionality of the control systems}

The subjects performed faster $\left(F(1.7,159.86)=230.08, p<0.001, \operatorname{Eta}^{2}=.710\right)$ in the clothespin relocation task with the hACS than with the other two interfaces (Figure 15 a). Post-hoc tests revealed the strong advantage of simultaneous control of both rotation and grasp for the hACS. Reliability of control measured by the number of dropped pins was particularly better with hACS (Figure $15 \mathrm{~b}$ ). An average of 2.8 pins were dropped using the hACS, while an average of 17.0 pins were dropped using SL and of 6.6 pins using CC.

In the box and blocks test with its simple grasp-release task with one DOF, there was a significant effect of the control interface $\left(F(1.71,75.27)=63.493, p<0.001, \operatorname{Eta}^{2}=.591\right)$. Post-hoc analysis revealed the same performance, i.e. same number of transported blocks, for the hACS and CC interface, while the performance with SL control was poorer (Figure 16). 

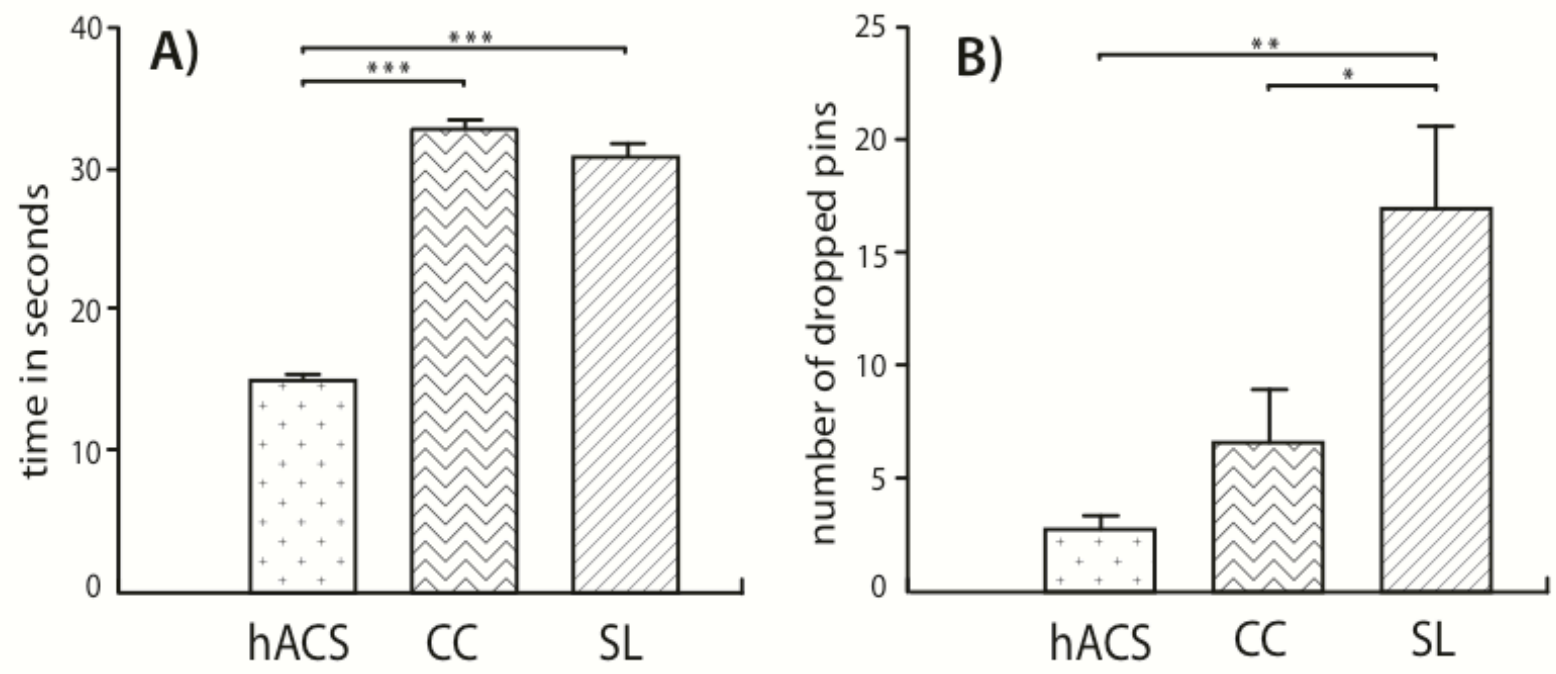

Figure 15) Speed and errors in ten able-bodied subjects in two-DOF clothespin relocation test (mean, SEM).

The hybrid ACS (hACS) performed significantly better than the two conventional control interfaces. They completed the task more rapidly (A) (pairwise comparison: hACS vs. CC $t(293)=24.38, p<0.001$; hACS vs. SL $t(299)=22.34, p<0.001)$ and dropped fewer pins (B). The statistical significance of the difference was greater for the comparison hACS versus SL (pairwise comparisons: ACS vs. SL $t(8)=4.18, p<0.01$, CC vs. SL $t(44)=10.76, p<0.05)$.

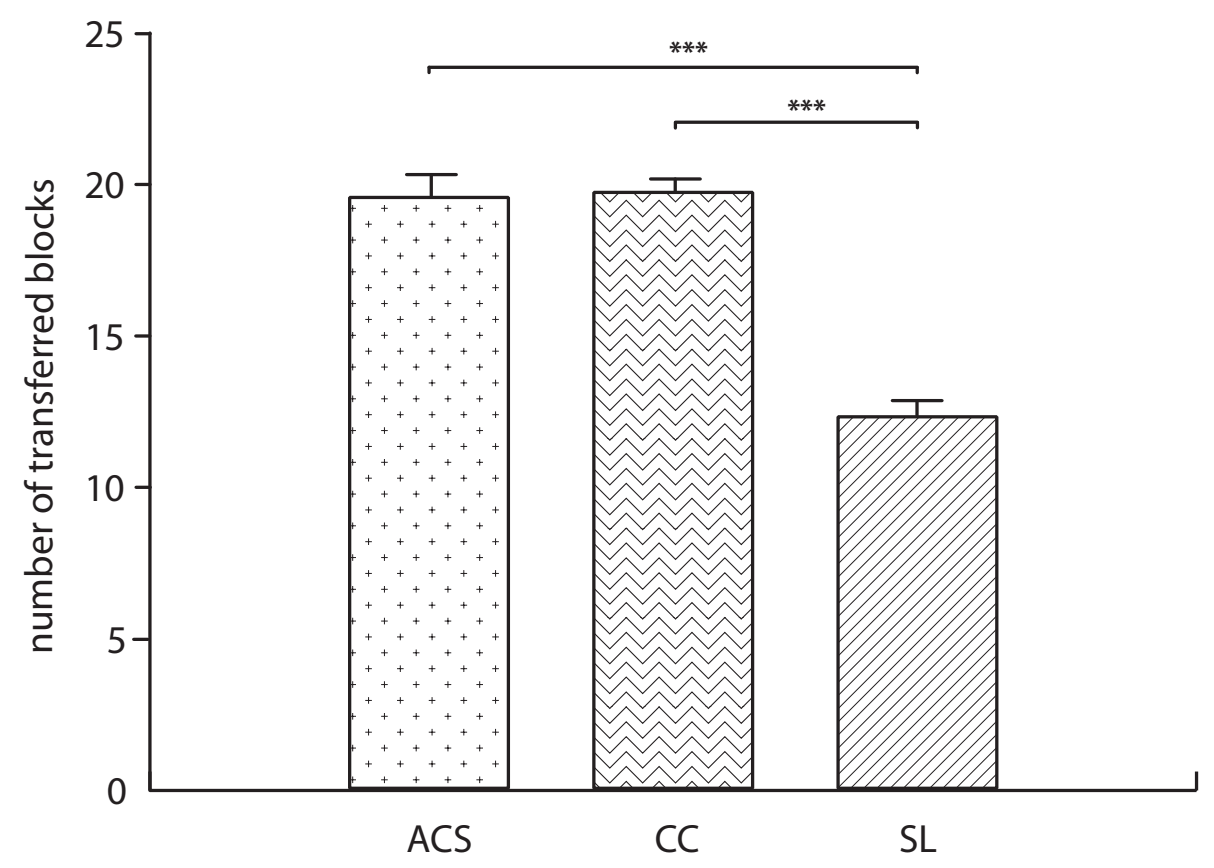

Figure 16) Results in one-DOF box and blocks test (mean, SEM).

hACS and CC performed equally well, while fewer blocks were dropped with SL. This indicates that when using hACS the subjects were able to inhibit involuntary ear movements to efficiently complete the task for which only the forearm signals for opening and closing the prosthesis were needed (pairwise comparisons: hACS vs. SL $t(49)=11.2, p<0.001$, CC vs. SL $t(44)=10.76, p<0.001)$. 


\subsubsection{Influence of arm position: robustness with hACS}

Arm position had a significant effect on completion time in the clothespin relocation task $\left(F(1.87,175.94)=30.1, p=.000, \mathrm{Eta}^{2}=.243\right.$, Figure 17), but the effect was not the same for the three control interfaces (control interface $\times$ arm position: $F(3.6,337.84)=2.5, p=.05$, $\mathrm{Eta}^{2}=.026$ ). We then normalized the completion time from the "high" arm position (that was the position with poorest performance) to the neutral "middle" arm position (setting it to 100\%). This delta was significantly greater for CC than hACS (t-tests), thus the impact of the arm position was less strong for the hACS (Figure 17). When using SL, precision also suffers from the high arm position, but not as much as when using CC.

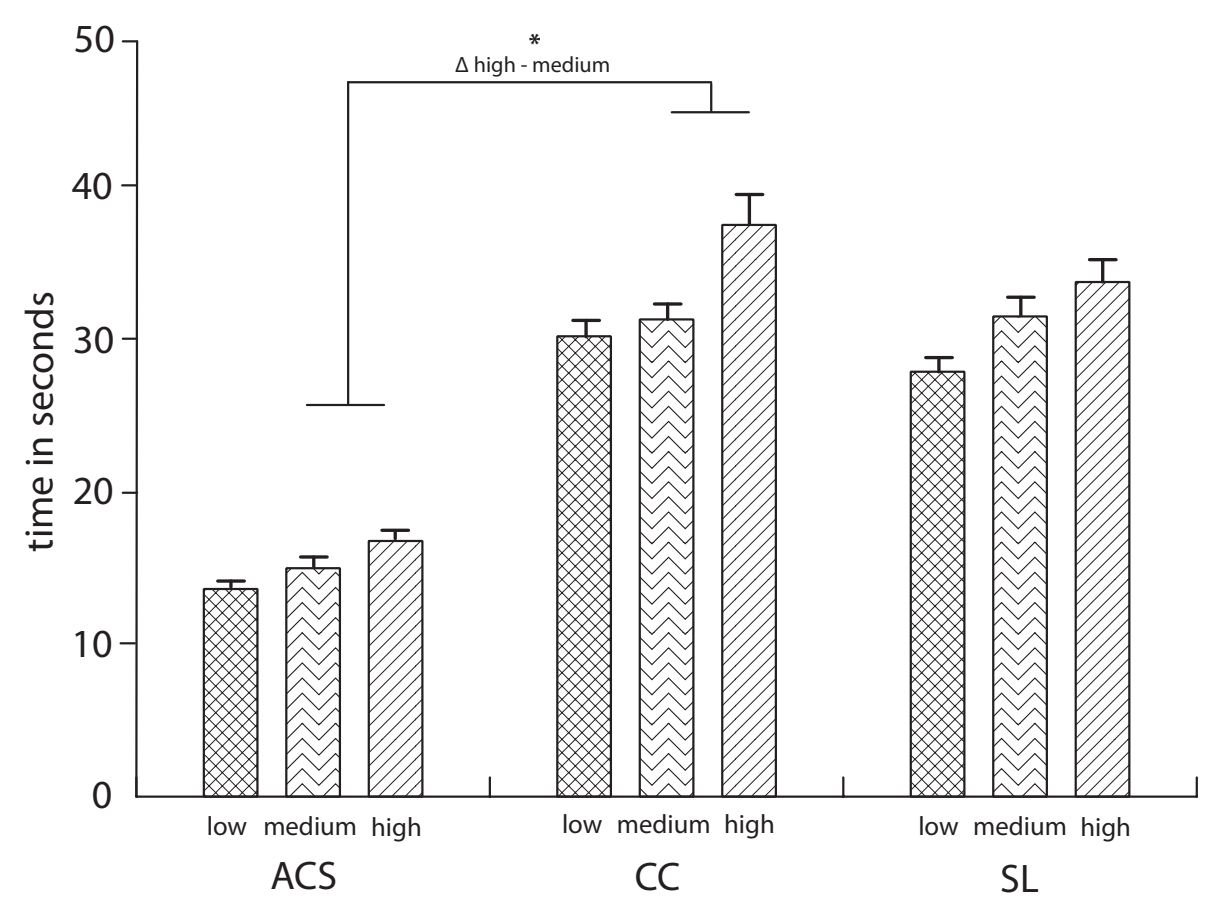

Figure 17) Influence of arm position on performance in the clothespin test (mean, SEM).

The arm position (low, middle, high) had a significant influence on performance in the clothespin relocation test. Test completion was most rapid with the arm in the lower position for all control interfaces, followed by the middle and high arm position. A comparison of the delta between the high and middle positions revealed that the negative effect of the arm position was less strong for the hACS than for the CC (pairwise comparison: $\Delta \mathrm{hACS}$ vs. $\Delta \mathrm{CC} t(98)=2.47, p<0.05$ ). Compared to SL, there was no significant difference either to CC or hACS. 


\subsubsection{Training effect}

Since none of the participants had any experience with prosthesis control, we expected significant training effects. Five runs of the clothespin relocation test were performed in each arm position using each of the three interfaces (block 1). The entire procedure was then repeated, giving two blocks of five runs per arm position. As expected, there was a difference between the two blocks $\left(F(1,44)=17.45, p=.000, \mathrm{Eta}^{2}=.284\right)$, albeit not for all prosthesis control conditions (time $\mathrm{x}$ control interface: $F(2,88)=4.79, p=.011$, $\left.\mathrm{Eta}^{2}=.098\right)$. Post-hoc t-tests revealed improved performance for hACS and SL in the second blocks but not for CC (Figure 18).

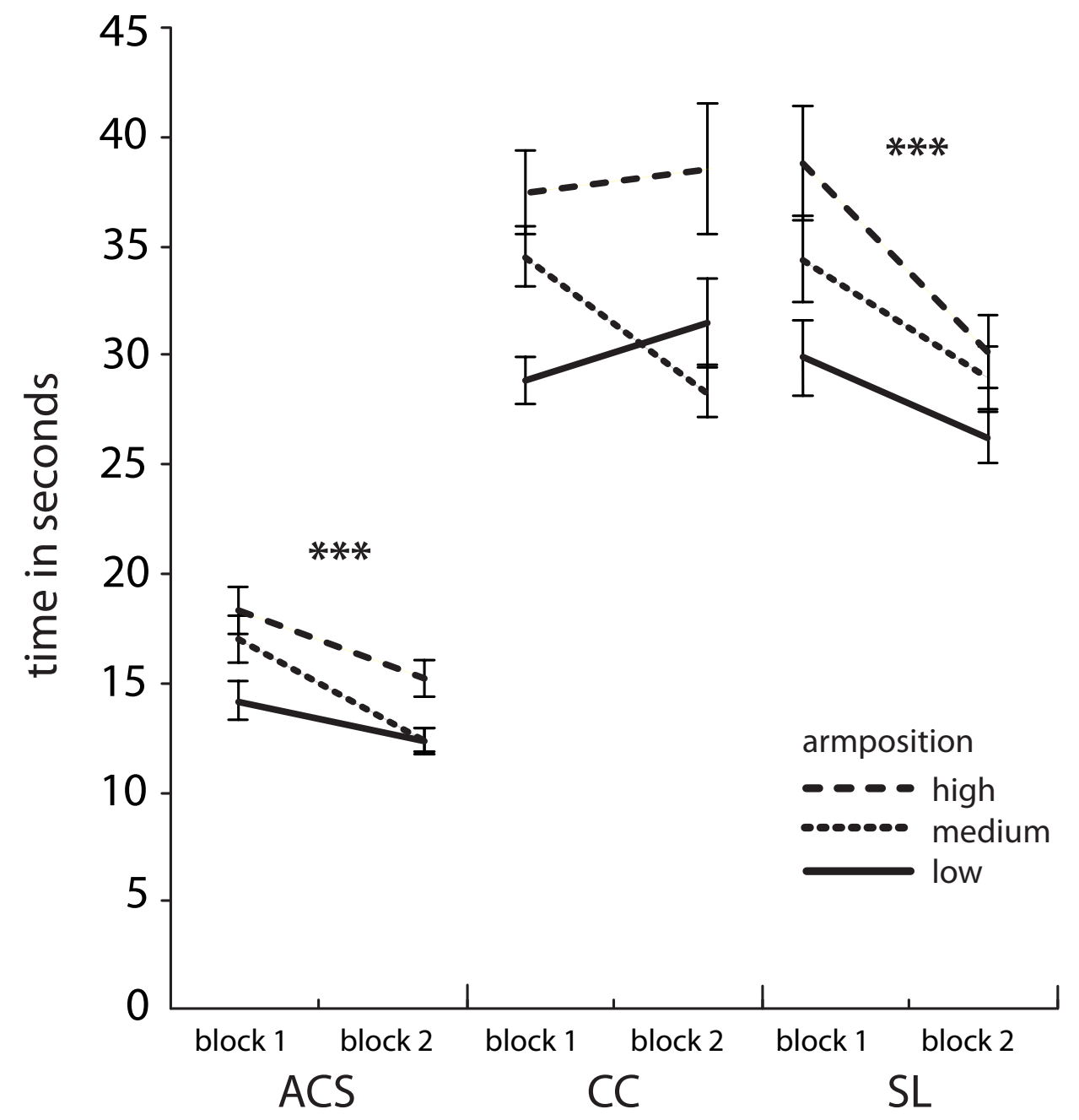

Figure 18) Training effect in the clothespin relocation test (mean, SEM).

A strong training effect was seen for hACS and SL. There was no significant training effect for CC (pairwise comparisons: hACS block 1 vs. hACS block $2 \mathrm{t}(149)=5.914, \mathrm{p}<0.001$; SL block 1 vs. SL block 2: $\mathrm{t}(149)=4.49$, $\mathrm{p}<0.001)$. The data suggest that this might be due to the more challenging high and low arm positions. 


\subsubsection{Results in a transradial amputee}

One 50-year-old man with a transradial amputation of the left forearm completed the same experimental sequence. In everyday life, he uses a hand prosthesis with a proportional open-close control using antagonistic forearm muscles. In his own words, he chose not to have a rotation because it was simpler for him to rotate the wrist with his contralateral hand than to use the established control interfaces, i.e. CC or SL. In the clothespin relocation test, he performed best with the hACS (Figure 19). His experience with myoelectric control is reflected in shorter completion times, particularly with CC and SL, compared to the able-bodied naïve subjects. Nonetheless, with the hACS, he was $60 \%$ faster than with CC and 30\% faster than with SL. In the box and blocks test, he transported more blocks with the hACS than with the SL (168\%, $\left.M_{h A C S}=27.2, M_{S L}=16.29\right)$ and only slightly fewer than with the CC $\left(M_{C C}=31.6,86 \%\right.$ of CC).

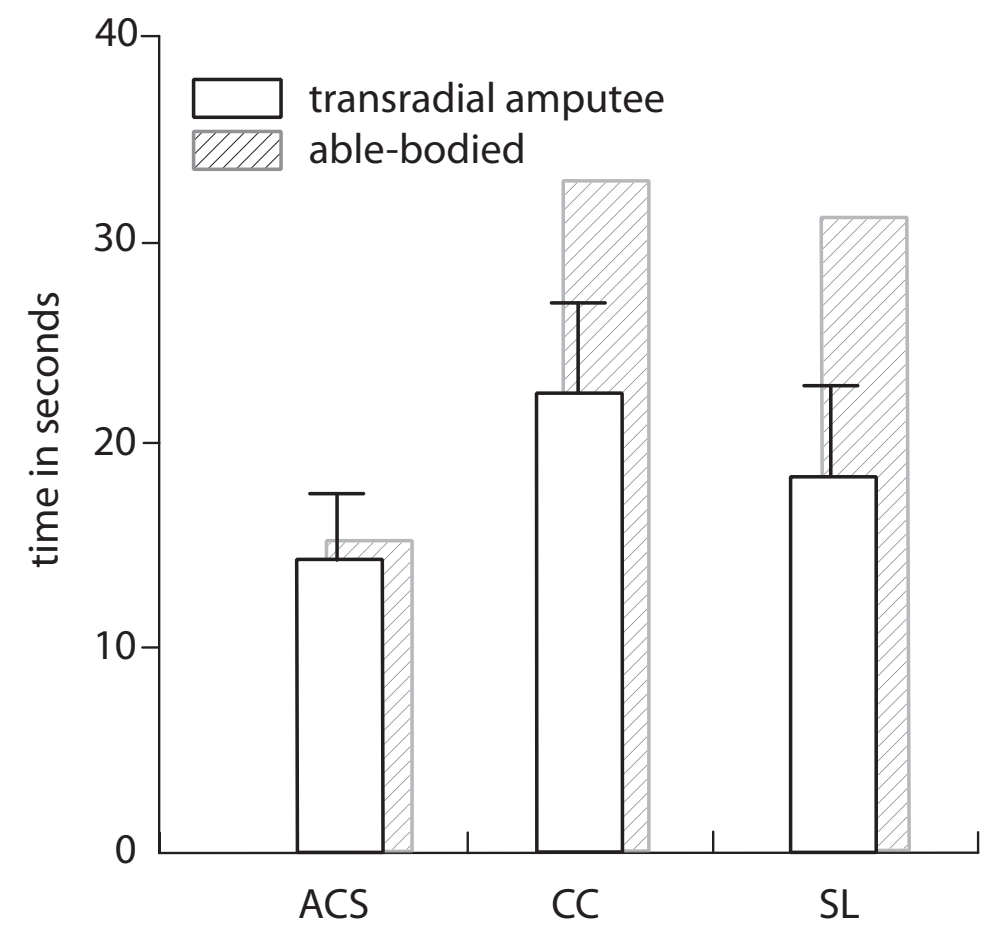

Figure 19) Results of the transradial amputee in the clothespin relocation test (mean, SD).

The amputee showed better performance with hACS than with the conventional control interfaces. The mean completion time with the other control interfaces was shorter than the means of the able-bodied sample (grey columns). 


\subsection{Discussion and conclusions}

In this proof-of-principle study, we presented a new concept of a hybrid myoelectric control interface that attributes a function to the formerly unexploited auricular muscles. The advantage of this approach is that both actuation DOFs can be controlled simultaneously and proportionally. In this study we compared this hybrid auricular control interface to two conventional control interfaces, CC and SL. The data from ablebodied subjects and from a forearm amputee with a hand prosthesis showed system performance superior to that of the two established control interface alternatives in standardized tests.

Subjects were able to relocate the clothespins more rapidly with the simultaneous control of rotation and grasp provided by the hACS. No interruptions for mode switching are required, which allows for very natural and fluent movements. As an example, rotating the hand back into the pronated position can be initiated while simultaneously opening the hand to release the pin on the vertical bar. Conversely, in CC, switching between the DOFs is slow and interrupts the fluid movement, while in SL, short pauses are required between contractions to permit detection of the slope.

The major drawback of both conventional control interfaces is their sequential nature of activation. SL attempts to minimize this problem by avoiding the tiresome and timeconsuming mode-switching of CC, and utilizes the slope of the EMG signal to classify different commands. However, our results did not show any difference between SL and CC in performance speed. The precise muscle activation and correct signal generation were presumably more complex in SL than with $\mathrm{CC}$, where the risk of incorrect activations was smaller. Learning to use SL was, therefore, more challenging but performance improved between the first and second block.

The hACS also allowed a greater precision, as measured by the number of dropped pins during the clothespin test. This quite remarkable accuracy of hACS suggests that the system is robust and has no trade-off between speed and accuracy.

Task completion time was shortest with hACS already in the first block that could indicate a more intuitive control than with the other interfaces. Training substantially improved performance with hACS and SL but not with CC, where the performance even worsened in the second block for the low and high arm positions (Figure 17). This is 
presumably due to the difficulty to reliably generate co-contractions in challenging arm positions. In these positions, the baseline muscle activation increases, as has been observed in elbow flexors (Howard, Hoit, Enoka, \& Hasan, 1986), thus possibly causing a tiring of the muscles that counteracts the learning effect.

The results of the box and blocks test documented the reliability of the hACS in situations in which the second DOF for rotation is not required and its activation should even be avoided. The subjects were able to avoid false ear muscle activation, which resulted in a performance similar to that with CC. On the other hand, with SL, fewer blocks were moved because in this control interface the grasp function is associated with slow contractions.

The acceptance rate of a control interface depends mainly on its controllability, which is determined by the accuracy of movement selection, the intuitiveness of actuating control, and the system response time (Englehart \& Hudgins, 2003). Our data revealed that the hACS is superior for the accurate selection of movements than the two other control systems. In addition, the hACS is programmed so that activation of the left ear muscles causes the hand to rotate counter clockwise ("left") while the right side induces the opposite motion. This leads to an intuitive grasp of the interface function, making it easy to use and learn. The fact that the ear muscles have no original function is an advantage over other proposed control options when it comes to interference with daily activities.

Finally, the hACS gives a fast response time with direct control of the two DOF. On the other hand, the logic of the two conventional control interfaces results in a delay of the desired output action (Amsuess et al., 2014). Other control alternatives invariably decrease the system response time (Englehart \& Hudgins, 2003; Mainardi \& Davalli, 2007). Adding an independent degree of freedom of control has previously resulted in affecting either the system response time, the intuitiveness of control (Amsuess et al., 2014), or the reliability of the interface (Hakonen, Piitulainen, \& Visala, 2015). In this study, the hACS has complied with all three characteristics that influence the acceptance rate, rendering it a viable alternative to established controls.

When compared to machine-learning-based control techniques, the hACS has the typical advantages of a direct control that maps the DOF to independent muscle signals. The performance of machine-learning-based control techniques is often greatly degraded by 
a change in arm position (Fougner et al., 2011; Ning Jiang et al., 2013). The small impact of arm position on hACS performance indicates a good robustness in daily use. This also indicates that effects of non-stationarities, which typically degrade the performance of machine-learning-based control interfaces over time (Vidovic et al., 2016), are also probably negligible.

Moreover, calibration of the hACS is simple; in contrast to time-consuming pattern learning, it only requires the adjustment of two gain factors and thresholds. To be suitable for daily use, the fine-wire electrodes could be replaced by implanted electrodes, such as a miniaturized version of the Implantable Myoelectric Sensor (Weir, Troyk, DeMichele, \& Kerns, 2005).

Although our results suggest that this interface is suitable for everyday use, a larger study with more prosthesis users is required to confirm this. The learnability of precise ear muscle activation that we have shown earlier (Schmalfuss et al., 2016) should also be replicated with a larger sample size.

In conclusion, we presented a novel control interface that allows the simultaneous and proportional control of a second DOF for myoelectric hand prostheses. In tasks that reflect daily life situations it responded more rapidly and more accurately than the two other most commonly used conventional myoelectric control techniques. We believe that it could be a viable add-on for lower-arm amputees with a prosthetic hand. 


\section{Neuronal effects of training the auricular control system for wheelchair control in subjects with tetraplegia.}

In the third study, the functionality of the ACS was tested for the first time in a clinical cohort of individuals with tetraplegia. The motor training involved in learning to use the ACS allowed us to investigate the nature of the post-SCI brain reorganization presented in the introduction. In chronic SCI, studies reported an expansion of the somatotopic representation of muscles in the primary motor cortex (Alkadhi et al., 2005; Curt, Bruehlmeier, Leenders, Roelcke, \& Dietz, 2002; Freund, Rothwell, Craggs, Thompson, \& Bestmann, 2011; Levy, Amassian, Traad, \& Cadwell, 1990; Lundell, Christensen, et al., 2011), as well as a shift of activation peaks either in direction of the de-efferented limb area (Bruehlmeier, 1998; Freund et al., 2013; Henderson et al., 2011; Mikulis et al., 2002) or, more posteriorly, in direction of the primary sensory cortex (Cramer et al., 2005; Green, Sora, Bialy, Ricamato, \& Thatcher, 1999; Turner et al., 2003). These changes might be influenced by the level of SCI lesion (Kokotilo et al., 2009).

The post-SCI brain reorganization can be maladaptive, e.g. lead to neuropathic pain, spasticity and phantom sensations (Moxon et al., 2014; Raineteau \& Schwab, 2001), and might further impair the motor performance (e.g. speed of movement, strength patterns)(Nardone et al., 2015; Wrigley et al., 2009). Progressive loss of grey matter in the somatosensory and motor cortex (Freund et al., 2013; Henderson et al., 2011; Jurkiewicz et al., 2007; Wrigley et al., 2009) is the harmful consequence of the inactivity of the affected brain parts (Freund, Weiskopf, et al., 2011). Although the functional relevance of this motor cortex reorganization is not fully understood (Moxon et al., 2014), it has been suggested that the loss of motor cortex function might be partly responsible for behavioral disability, and that it is important to "harness plasticity" (Silva et al., 2014) in order to exploit the full recovery potential of individuals with SCI. Whether this plasticity can be actively targeted in order to prevent potentially negative consequences is still unclear, but there are indications that such an intervention could be beneficial. For example, when unoccupied areas in the sensory cortex are invaded by neighboring areas and thus assume a new function, the observed grey matter loss is of a lesser magnitude (Henderson et al., 2011). Given the scarce information about activitydependent plasticity in individuals with SCI, it is important to increase the knowledge within this field (Kokotilo et al., 2009). This might not only help preserve affected brain 
areas, but could also help to optimize physiotherapeutic care and improve motoric output (Dunlop, 2008).

Therefore the aim of the present study was to provide further evidence of the functionality of the ACS in a clinical sample and with longer training times. Using fMRI and robot-guided TMS mapping, we tested the impact of motor training, i.e. the use of the PAMs for wheelchair control, on the post-SCI motor cortex reorganization, i.e. the expansion and localization of auricular motor maps.

This study is being prepared for publication under the title "Targeting brain reorganization: motor cortex changes in tetraplegic subjects after training with auricular wheelchair control".

\subsection{Methods}

\subsubsection{Study design}

Ten participants were recruited for the study (see Table 1). One dropped out after three days for reasons not related to our study. Nine participants $(39.7 \pm 16.5 \mathrm{yrs}$, mean \pm SD) trained auricular control of an electric wheelchair for one hour daily on 12 consecutive days. The level of the spinal cord injury (lesion level) ranged from C6 to C4. Daily assessments were performed to measure the training effect on e.g. unilateral auricular activation and actual wheelchair driving. Before and after training, PAM representations and the finger area (FT) as a reference of the de-efferented limb were mapped on the motor cortex using fMRI and TMS (day 0 = pre-training; day 13 = post-training).

All participants gave their written consent to the study. If unable to write, a designated representative signed for them. The study design was approved by the ethics committee of the University of Göttingen and was in accordance with the declaration of Helsinki. During the study, all participants were hospitalized in cooperating rehabilitation centers where they took part in the normal rehabilitation routine.

\subsubsection{Software-based training and testing of control capacity of the auricular muscles}

The set up of the ACS (Figure 20) and EMG signal recording are described in detail in chapters 2.1.1 and 2.1.2. The participants trained voluntary and unilateral PAM 
activation using visual feedback and video games on computer software (see chapter 2.1.5). When they had successfully completed a game of their choice, the computer training was abandoned in favor of actual wheelchair driving.

Prior to each training session, the signal strength was calibrated for each participant with an automated routine using the mean of three maximum contractions per side. A minimum activation threshold was set manually. Unilateral activation was then tested: the participants were instructed to activate the PAM of only one side for three seconds, three times in succession. In order to attain a $100 \%$ success rate, the intended side had to be activated to its individual maximum, without simultaneously activating the other side. Less strong contractions or co-activations gave lower scores. Performance (completion time and path length) in a virtual obstacle course was then tested. Steering signals for this virtual wheelchair were the same as for the real wheelchair (see below).

\begin{tabular}{|lllllll||}
\hline Subject & Age & Lesion level & $\begin{array}{l}\text { Time since } \\
\text { lesion }\end{array}$ & $\begin{array}{l}\text { Usual } \\
\text { wheelchair } \\
\text { control }\end{array}$ & $\begin{array}{l}\text { Able to } \\
\text { activate } \\
\text { PAM? }\end{array}$ & Remarks \\
\hline S 1 & 19 & C4 & 5 & joystick & Yes & \\
S 2 & 50 & C6 & 2 & manual & No & \\
S 3 & 43 & C5 & 22 & manual & Yes & \\
S 4 & 27 & C4 & 4 & chin control & No & drop out \\
S 5 & 57 & C6 & 5 & manual & No & \\
S 6 & 54 & C4 & 2 & joystick & No & \\
S 7 & 67 & C5 & 7 & chin control & No & \\
S 8 & 28 & C4 & 10 & chin control & No & \\
S 9 & 27 & C4 & 7 & chin control & No & \\
S 10 & 25 & C5 & 2 & joystick & No & \\
\hline
\end{tabular}

Table 1) Clinical information on the ten study participants.

Ten subjects $(39.7 \pm 16.5 \mathrm{yrs}$, mean $\pm \mathrm{SD}$ ) participated in the study. One subject dropped out due to illness not related to the study. Five participants had a C4 lesion level, three C5 and one C6. The mean time since the SCI lesion happened was 6 years and 1 month (SD 6 y $3 \mathrm{~m}$ ). The wheelchair controls used by our participants were manual (using the push rims), joystick with special T-handle or chin control. Two participants were able to activate their bilateral PAM prior to training. The others were naïve but managed to produce PAM contractions with visual EMG-feedback before the first fMRI scans. 

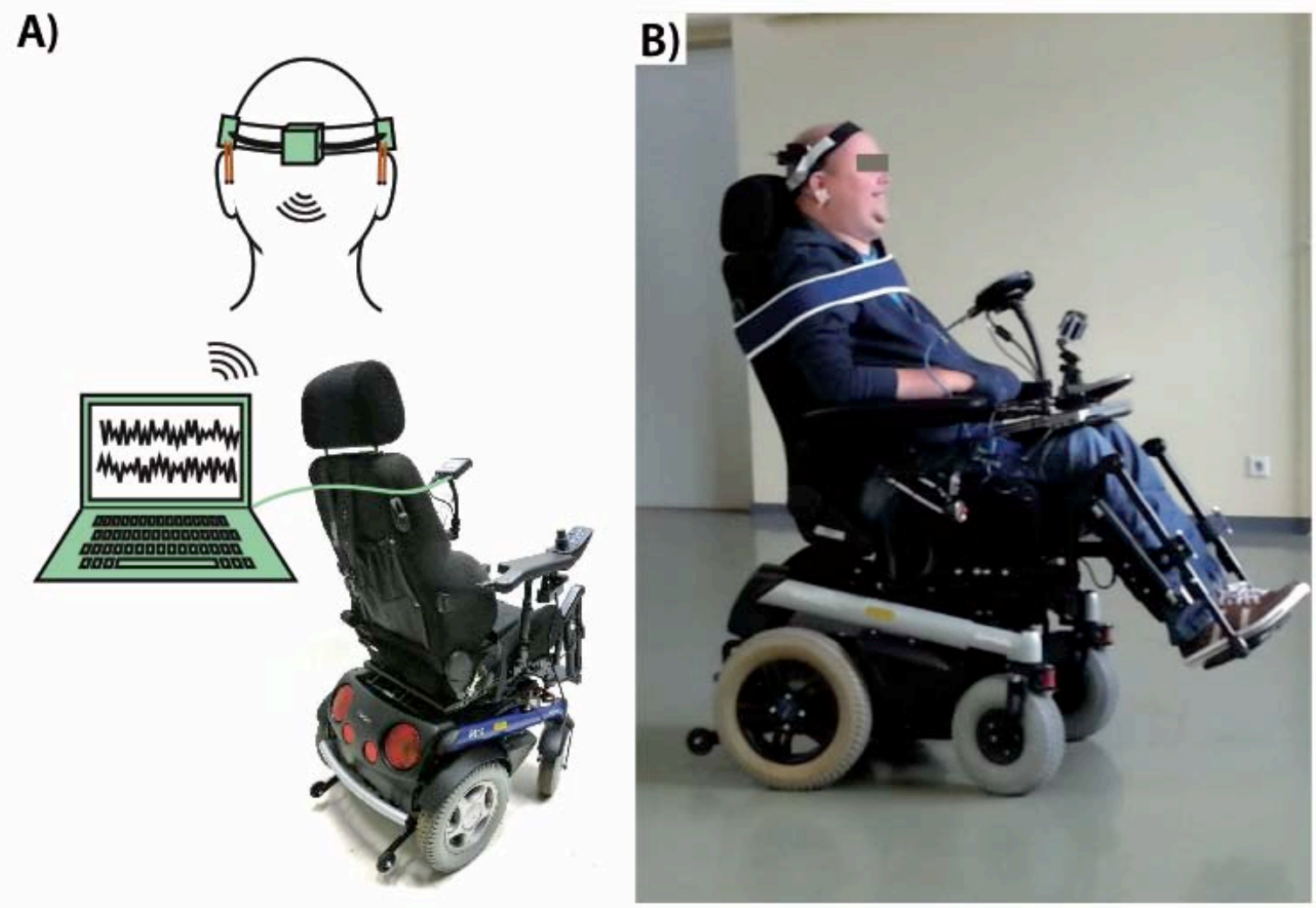

Figure 20) Wheelchair set-up with the auricular control system (ACS).

For recording the EMG, fine-wire electrodes were inserted subcutaneously over the posterior auricular muscle (PAM) and the pinna (A). The electrodes were connected to an amplifier that was mounted on headset. The EMG signals are transmitted wirelessly to a computer, where they are processed and converted into wheelchair steering commands, which are then transmitted by cable to the wheelchair. A subject with a lesion at the $\mathrm{C} 4$ level controlled the electric wheelchair solely with signals generated by his auricular muscles (B). The computer was positioned on his lap and connected to the external joystick interface. In order to ensure stability, the person was fastened to the wheelchair with an elastic band.

\subsubsection{Training and testing of the ACS applied to wheelchair control}

Driving performance with the ACS was assessed by measuring the time required to complete a real obstacle course that was arranged in the same pattern as the virtual obstacle course. The ideal path was ca. $23 \mathrm{~m}$ long and included three right and three left turns (see chapter 2.1.7, Figure 9). In addition, the participants had to complete a 12meter-long slalom course with three obstacles. In the event of a collision in either task (i.e. crossing the borders), the timer was stopped, and the wheelchair was manually placed back on track. In some cases, the testing routine had to be reduced to accommodate the condition of the participant (e.g. not feeling well, tired etc.). After finishing the driving tests, the remaining time was used for training under the 
supervision of the PI. The wheelchair commands are described in detail in chapters 2.1.3.

\subsubsection{User evaluation of the ACS for wheelchair control}

After completion of the study, the participants rated the perceived workload for using the ACS with the NASA TLX (Task Load Index, e.g. mental, physical and temporal demand, performance, effort and frustration, ten-point rating scale from $0=$ very little, to 100 = very high (Hart \& Staveland,1988; Rubio, Diaz, Martin, \& Puente, 2004)). The ACS was evaluated with the QUEST scale, which rated safety, comfort, ease of use, and learnability on a five-point scale from 1 = "not satisfied at all" to $5=$ "very satisfied", and with our own questionnaire (see supplementary material, p. 104) with a four-point scale from 1 = "not at all" to 4 = "a lot".

\subsubsection{Acquisition and analysis of functional imagining data}

Visual EMG feedback was performed prior to the first fMRI session to ensure isolated PAM activation during scanning. Surface EMG electrodes were fixed on the corrugator supercilii, the zygomaticus major and on the PAM on the right facial half. All participants were able to produce PAM contractions with minimal co-contraction of other facial muscles (mean cheek co-activation $=9.5 \%$ of cheek maximum, $\mathrm{SD}=4.6 \%$; mean eyebrow co-activation $=23 \%, \mathrm{SD}=13 \%)$. Two participants required a longer training period in order to avoid confounds from facial co-activations in the MRI scanner.

Imaging data was acquired at 3T in a Magnetom TIM Trio scanner (Siemens Healthcare, Erlangen, Germany) using a standard 32-channel phased-array head coil. Participants lay supine in the scanner with earplugs for noise protection. The head was stabilized with foam pads and the arms were stabilized with bands at the side of the trunk. In each session data collection lasted approximately 45 minutes.

First a T1-weighted 3D turbo fast, low-angle shot (FLASH), anatomical image at $1 \mathrm{~mm}^{3}$ isotropic resolution (repetition time (TR): $2250 \mathrm{~ms}$, inversion time: $900 \mathrm{~ms}$, echo time (TE): $3.26 \mathrm{~ms}$, and flip angle: $9^{\circ}$ ) was acquired. Four runs of functional blood-oxygenlevel-dependent (BOLD) contrast were then recorded using a T2*-weighted gradientecho echo-planar imaging (EPI) technique with simultaneous multi-slice acquisition (multiband factor: 3). Functional images had an in-plane resolution of $2 \times 2 \mathrm{~mm}^{2} .36$ 
slices of $2 \mathrm{~mm}$ thickness with a $0.2 \mathrm{~mm}$ gap between slices were acquired (253 repetitions with TR: $1050 \mathrm{~ms}$, TE: $36 \mathrm{~ms}$, flip angle: 50, acquisition matrix: 128x96, partial fourier $7 / 8$ ). In these runs, the participant performed blocks of bilateral PAM activation, imaginary bilateral finger tapping of both index fingers against the thumb (FT) and another PAM activation each lasting 4 minutes and 25 seconds. Each run consisted of 11 blocks of rest $(14 \mathrm{TR}=14.7 \mathrm{~s})$ and 10 blocks of the active condition ( 9 $\mathrm{TR}=9.45 \mathrm{~s}$ ). The beginning and the end of active blocks were cued by a green or red disk, respectively, on a screen at the end of the scanner tunnel which subjects could see in a mirror mounted above their head. The participants were carefully instructed to avoid any head movement or additional facial movements during PAM activation.

For data analysis, BrainVoyager QX (Brain Innovation, Maastricht, The Netherland), Matlab (MathWorks, Natick, MA) and the NeuroElf toolbox (neuroelf.net) were used. Data was preprocessed with standard parameters, using slice-scan time correction, 3D motion correction and temporal high-pass filtering (with a cutoff frequency of 2 cycles per run, i.e. $0.00765 \mathrm{~Hz}$ ). The functional data was co-aligned to the anatomical T1 dataset of each individual, transformed into Talairach space and spatially smoothed with a $5 \mathrm{~mm}$ FWHM Gaussian filter. High motion data points were identified using framewise displacement (FD) as described by (Power et al., 2014) (FD cutoff: 0.5mm). All data points with high motion plus the one preceding and the two following data points were excluded from the analysis. In addition, the estimated motion parameters (3 translations and 3 rotations) and the FD were included in the general linear model (GLM) models as predictors of no interest. The first four images of every functional run were discarded to allow for T1 equilibrium.

For the individual analysis of the functional data, periods of active task (movement or imagined movement) were contrasted with periods of rest. We calculated a GLM with boxcar predictors for task convolved with BV's standard 2-gamma hemodynamic response function (hrf) plus motion confounds. Data was corrected for temporal autocorrelation (AR-1).

Regions of interest (ROI) were marked manually on an inflated representation of the cortical surface of each participant's head. The area of the primary motor cortex (M1) was identified as the area between the depth of the central sulcus posteriorly, the crown of the precentral gyrus anteriorly, the lobus paracentralis medially and the lateral sulcus laterally. In addition, the supplementary motor area (SMA) was marked with the 
posterior border at the lobus paracentralis, and the anterior border at the vertical line that goes through the anterior commissure (Chainay et al., 2004).

We calculated a first set of ROI activation with a threshold at $p=0.05$ (Bonferronicorrected for amount of activated voxels) which was automatically reduced until at least 100 active voxels were detected. Since activation sizes diminished in the post-training session, thresholds decreased between sessions. Variable thresholds make activation sizes difficult to compare between sessions (Jurkiewicz et al., 2007; Mikulis et al., 2002). Therefore, we extracted the threshold from the post-training session for each participant. In another ROI analysis, these thresholds (mean $t(8)=4.379(0.517$ SD) were used for both sessions. Total sizes of activation clusters and center of gravities (COG) from the largest cluster were extracted for each participant, hemisphere and task. Additionally, we calculated a conjunction analysis of those areas that were activated during both PAM and FT movement with a random effect model (threshold at $t(8)>3.5$ ) for pre- and post-training sessions. For illustration purposes, we calculated a wholebrain group analysis with a random effect model (PAM vs. rest, FT vs. rest). We adopted a common threshold for all tasks at $p<0.00395$, corresponding to $t(8)>4.00$.

We compared the results from the pre-training session to fMRI data from able-bodied subjects acquired previously by our group ( $n=13)$ (Meincke et al., n.d.). The PAM task was the same as in our fMRI acquisition, but the finger task was different, involving a bilateral lateral spread of the index finger. Cluster sizes and COGs of PAM and executed FT activation during fMRI (mean threshold $t(12)=4.8$ ) were used for comparison. For more details on image acquisition, see Meincke et al., n.d..

\subsubsection{Robotic arm-controlled and image-guided TMS mapping procedure}

Two TMS mapping sessions were performed (pre- and post-training) in each participant using a Magstim $200^{2}$ magnetic pulse stimulator with a figure-of-eight coil $(70 \mathrm{~mm}$, Magstim Company, Whitland, UK, magnetic field strength at maximum stimulator output intensity (MSO) $2.2 \mathrm{~T}$ ).

The coil was positioned tangentially over the scalp with $45^{\circ}$ rotation to the sagittal plane using an image-guided TMS mapping technology. An Adept Viper s850 robotic arm (Adept Technology, Inc. Livermore, CA, USA) controlled by a robot navigation software (ANT, Enschede, Netherlands) running on a standard PC positioned the coil. The 
software calculated a 3D head model for each participant based on MRI data (3 T scanner, Magnetom Trio, Siemens, Erlangen, Germany). Feedback from a NDI Polaris Vicra infrared tracking camera allowed for compensation of head movements, thereby ensuring an exact positioning of the TMS coil (position error: $\pm 0.02 \mathrm{~mm}$ ). The navigation software was networked with the EMG recording system. Here, a script controlled the experiment, including online randomization of stimulation targets, triggering of the stimulation, and retesting of rejected grid points.

The center of a 7 x 7 grid with $1 \mathrm{~cm}$ distance was placed over the PAM hotspot identified on the left hemisphere ( $6 \mathrm{~cm}$ medial to Cz from the EEG 10-20 system, $2 \mathrm{~cm}$ frontal) in previous studies by our group (Meincke et al., n.d.). We chose a large grid in order to account for possible cortical reorganization and activation shifts following SCI (Freund, Weiskopf, et al., 2011; Green et al., 1998; Karl, Birbaumer, Lutzenberger, Cohen, \& Flor, 2001). TMS pulses at 65\% maximum stimulator output were applied at each grid point (a total of eight stimuli in a randomized order). Depending on the duration of the robotic arm movement, the interstimulus interval varied between 4 and 8 seconds.

For EMG of the PAM, the previously placed fine-wire electrodes were connected to the EMG recording system. TMS of the PAM was applied during pre-innervation (at least $20 \%$ of the individual maximum MEP size), which was cued by an acoustic signal $1 \mathrm{~s}$ before the TMS pulse. This technique has been shown to produce more reliable MEPs in small muscles like the PAM. If the participant failed to pre-innervate, an acoustic feedback was given and the target grid point was repeated later on.

The entire procedure, including preparation, lasted around three to four hours per session. In order to avoid exhausting the participant, only the left hemisphere was mapped. The stimulation protocol described here was adapted following the second participant because we found that our tetraplegia patients were unable to endure the initial, longer stimulation protocol due to physical fatigue and positioning problems.

Data analysis was done using Matlab (Math Works, Inc., Natick, MA, U.S.A.) and FSL FNIRT and FLIRT). The center of gravity (COG) was calculated from the PAM MEPs according to (Meincke et al., n.d.). 


\subsubsection{Statistical analysis of the data}

Behavioral data was normally distributed and analyzed with paired-samples t-tests. Kolmogorov-Smirnov tests showed that the fMRI and TMS data were not normally distributed, and we used the non-parametric Wilcoxon signed-rank test for comparisons and the non-parametric Spearman-Rho for correlations. Comparisons with the data of the able-bodied subjects were performed with the non-parametric Kruskal-Wallis-test for independent samples.

\subsection{Results}

\subsubsection{Training effects on voluntary control over the PAM}

The participants improved their ability to activate each PAM separately (see Figure 21 a) from $49 \%$ in the pre-training session to $78 \%$ post-training (pairwise comparison prevs. post-training: $t(8)=-4.465, p<0.01)$. Those with a higher lesion level had better unilateral activation in the pre-training session $(r=0.820, p=0.007)$, but this difference was attenuated through training $(r=0.484, p=0.186)$. In addition, individuals with a higher lesion level had a stronger learning effect in unilateral activation $(r=0.748$, $p=0.02$ ). The time needed to complete the virtual obstacle course decreased from 5.9 minutes in the pre-training session to 2.7 minutes in the post-training session (pairwise comparison: $t(7)=5.272, p=0.001$; see Figure 21 b). The average path length was reduced from 3144 pixel pre-training to 2137 post-training (pairwise comparison: $t(7)=6.095, p<0.001)$. We found a significant correlation between higher lesion levels and better results in the virtual obstacle course post-training (path length: $r=0.783$; $p=0.013$; completion time: $r=0.671 ; p=0.048$ ). This correlation was not significant pretraining (path length: $r=0.224 ; p=0.563$; completion time: $r=0.410 ; p=0.273$ ).

\subsubsection{Training effects on wheelchair driving using the ACS}

As in the virtual course, the ability of all participants to control a real wheelchair improved with training. The average time to complete the slalom decreased from $46 \mathrm{~s}$ to $23 \mathrm{~s}$ (pairwise comparisons pre- vs. post-training: $t(6)=6.321, p=0.001$; see Figure $21 \mathrm{c}$ ). Completing the complex real obstacle course took an average of $183 \mathrm{sec}$ on the first testing but decreased to $84 \mathrm{sec}$ (pairwise comparisons pre- vs. post-training: $t(8)=4.318$, $p=0.003$; see Figure $21 \mathrm{~d}$ ). Higher lesion levels correlated with better performance in the 
pre-training real obstacle course $(r=0.674, p=0.047)$ but this correlation was not detectable after training $(r=0.373, p=0.323)$. The higher lesion levels were also significantly correlated with better slalom performance after training $(r=0.671$, $p=0.048)$, but not before $(r=0.262, p=0.496)$.
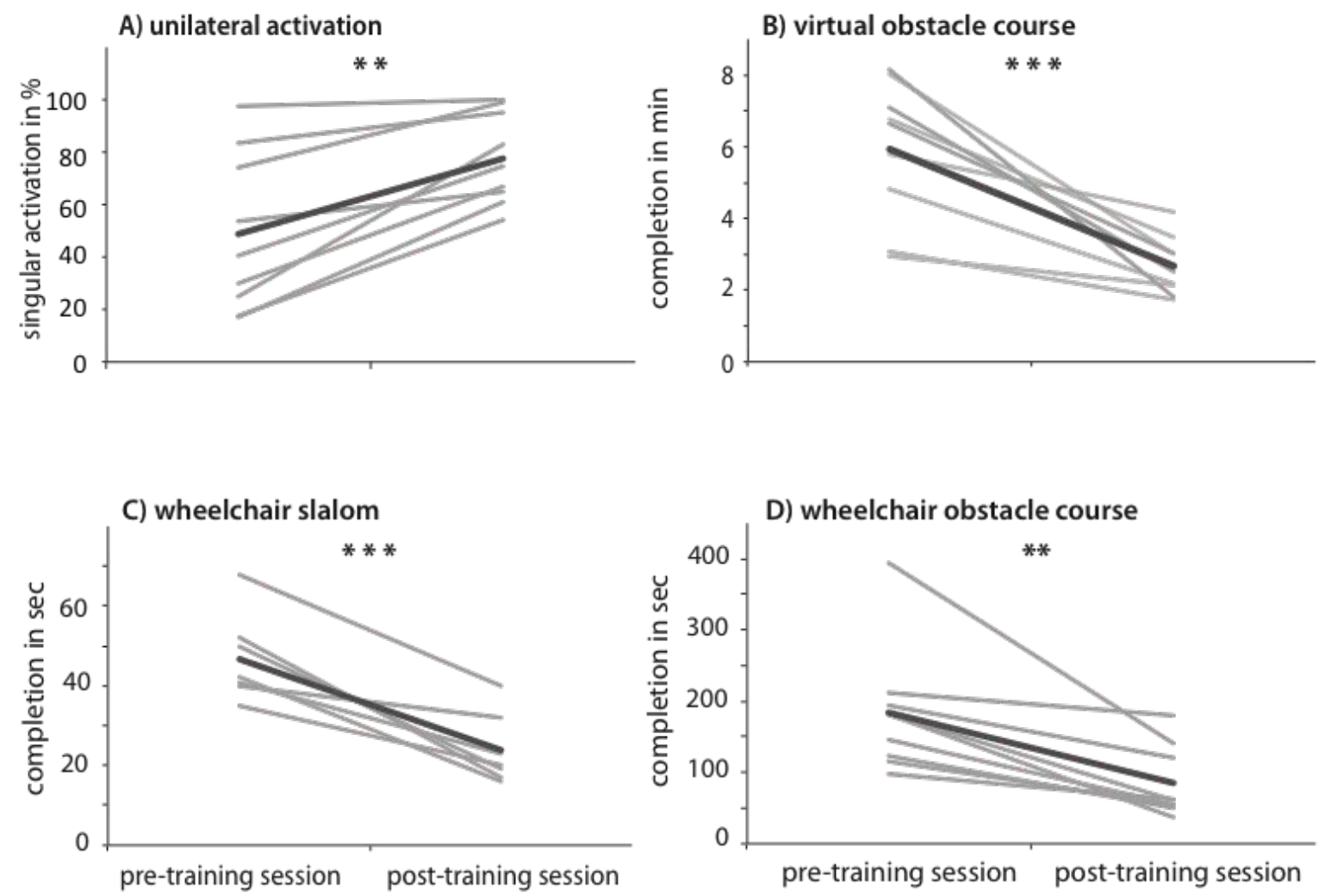

Figure 21) Effects of training on the control capacity of the PAM.

Individual results (light grey lines) and group means (dark grey) from pre-training and post-training sessions. The participants improved their ability to activate their auricular muscles unilaterally, i.e. to distinguish between left and right side (a). They completed the virtual obstacle course faster after training (b). The participants improved their ability to navigate a real wheelchair through a 12-meter slalom course with three obstacles only using their auricular muscles for steering (c). The complex, real obstacle course was completed faster in the real wheelchair after training (d). Significant differences are marked ** for $\mathrm{p}<0.01$ and ${ }^{* * *}$ for $\mathrm{p}<0.001$.

\subsubsection{User evaluation of the ACS for wheelchair control}

The NASA TLX (answering scale: $0=$ very low $-100=$ very high) revealed a low general workload for the group $(M=29)$. The mental demands for using the ACS (mental demand, effort and frustration) were judged as slightly higher $(M=37)$ than the physical demands $(M=21)$. Subjects in general rated their success as good $(M=06$, note that the answering scale is switched in this item: $0=\operatorname{good}-100=$ poor). 
The participants gave high scores for ACS in the QUEST (average score of 4.14 of the maximal 5), as well as in our own questionnaire (average score of 3.3 of maximal 4). In the QUEST, the participants rated the system as easy to learn ( $M=4.6$ out of 5$)$ and easy to use $(M=4.3)$. They were quite satisfied with safety, comfort and reliability of the system $(M=4.0)$. In our own questionnaire, the participants were content with the maneuvering characteristics of the ACS (e.g. wide turns, precision, response time; $M=3.5$ out of 4). They judged it to be ready for use in daily life $(M=3.6)$.

\subsubsection{Activation cluster size and centers of gravity during motor tasks in fMRI}

Mean coordinates of centers of gravity (COGs) of brain activations during PAM movement and imaginary finger tapping was not shifted significantly by training (see Table 2, Figure 22). However, we found a significant difference between the location of the PAM COG and the FT COG in the pre-training session, which had disappeared in the post-training session. This was true for the $\mathrm{x}$ (left hemisphere (LH) pre-training: $Z=-$ 2.073, $p=0.038$; LH post-training: $Z=-0.889, p=0.374$; right hemisphere (RH) pretraining: $Z=-2.666, p=0.008$; RH post-training: $Z=-1.244, p=0.214$ ) and y coordinates (LH pre-training: $Z=-2.666, p=0.008$; LH post-training: $Z=-1.599, p=0.110$; RH pre-training: $Z=-2.666, p=0.008$; RH post-training: $Z=-1.599, p=0.110$ ).

The size of the activations did not differ between the hemispheres in any of the tasks (all $p>0,1$; see Table 2, Figure 23, Figure 24), but it diminished with training. This reduction was significant for the PAM area in the left hemisphere $(Z=-2.429, p=0.015)$ but not in the right $(p>0.5)$. On an individual level, area size decreased in eight of the nine participants during PAM activation in the left hemisphere, while in the right hemisphere this was observed in only four of the nine (Figure 25). Here, five individuals activated slightly increased areas (mean growth: 892 vs. mean reduction -3282). The FT area in the right hemisphere also decreased significantly $(Z=-2.073, p=0.038)$ and showed a trend for the left hemisphere $(p=0.08)$.

Of interest is the observation that higher lesion levels correlated with greater M1 activations during PAM movement in the pre-training session (LH pre-training: $r=0.820$, $p=0.007$, Figure $26 \mathrm{~A}$ ), and that this effect was reversed by training, albeit not significantly (LH post-training: $r=-0.186 p=0.631$ ). The same proportion was present in the right hemisphere but the initial correlation was less strong (RH pre-training: 
$r=0.459, p=0.09$; RH post-training: $r=-0.261, p=0.498$ ). Higher lesions appeared to also be correlated with greater FT activation in the pre-training session but the reduction after training was less pronounced (LH pre-training: $r=0.745, p=0.021$, LH post-training: $r=0.298, p=0.436$; RH pre-training: $r=0.783, p=0.013$, RH post-training: $r=0.186$, $p=0.631)$.

Conjunction analysis revealed the location and extent of overlapping areas that were activated in both PAM and FT. Mean coordinates for the right hemisphere were $\mathrm{x}=36.2$ (9.5 SD); $y=-20.9$ (5.6 SD); $z=54.6$ (10.6 SD) for pre-training and $x=36$ (3.7 SD); $y=-19.5$ (7 SD); $z=55.2$ (3.6 SD) for post-training. For the left hemisphere, coordinates for conjunction activation were $\mathrm{x}=-34.6$ (6.3 SD); $\mathrm{y}=-20.1$ (7.5 SD); $\mathrm{z}=57.7$ (4.7 SD) for pretraining and $x=-34.1$ (10.6 SD); $y=-19.9$ (8 SD); $z=55.5$ (11.4 SD) for post-training. There was no significant shift in the mean COG coordinates after training. The size of the shared area decreased on average, but since the area increased in size in some individuals, there was no significant difference between sessions.

Seven of the nine participants had activations on the medial part of the primary motor cortex (i.e. leg area) when activating the PAM before training (Figure 23, Figure 24). However, after training, six of the nine no longer recruited this area. Only one individual had consistent bilateral activations after training, while two had only right or left activation. The picture was more diffuse for FT: four participants exhibited FT activations in the medial part of M1 in the pre-training session, and five post-training. However, size of activation decreased considerably (Figure 23).

Activation in the more frontal part of the medial wall, the SMA, was found for all tasks in all participants. COGs did not shift significantly between testing sessions. Activation size decreased in the second testing for the FT (RH: $Z=-2.310, p=0.021$ ) and almost significantly for the PAM (RH: $Z=-1.955, p=0.051$ ). In both tasks, cluster size reductions in the left hemisphere were not significant $(p>0.3)$. 


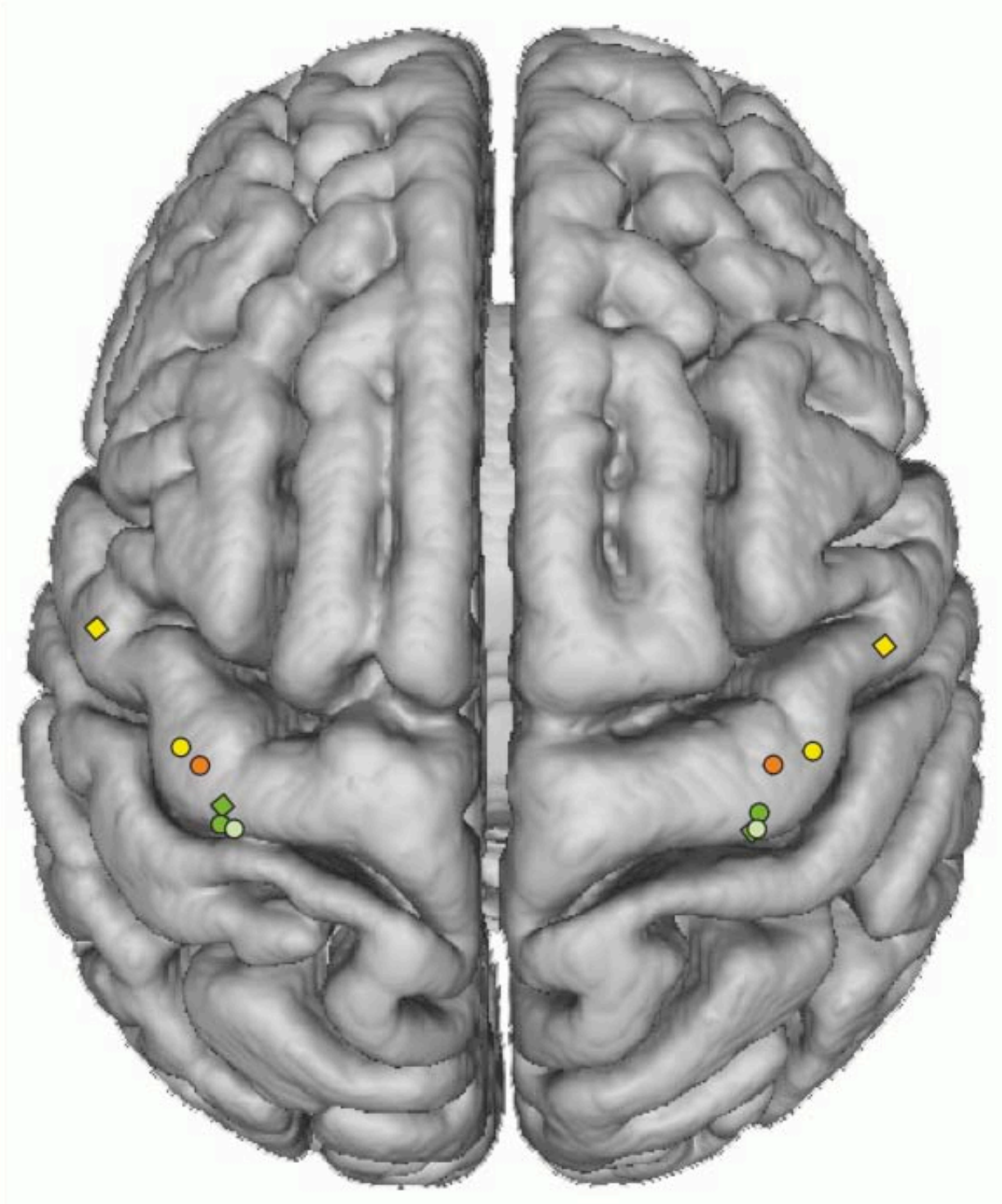

Figure 22) Centers of gravity (COG) of PAM and FT fMRI representations of SCI and able-bodied subjects.

Superior view of mean COGs of SCI subjects (circles) and able-bodied subjects (squares) projected on a 3D MNI standard brain. Coordinates were converted to MNI space using http://sprout022.sprout.yale.edu/mni2tal/mni2tal.html60. Pre-training PAM COGs are yellow, and the post-training results are orange. Finger tapping (FT) COGs are dark green (pre-training) and light green (post-training). Pre-training PAM COGs reveal a shift of the PAM COG into the de-efferented hand area in SCI-subjects compared to healthy subjects. Training moved the PAM COG further towards the COG of the hand area, which remained largely unchanged by training. 


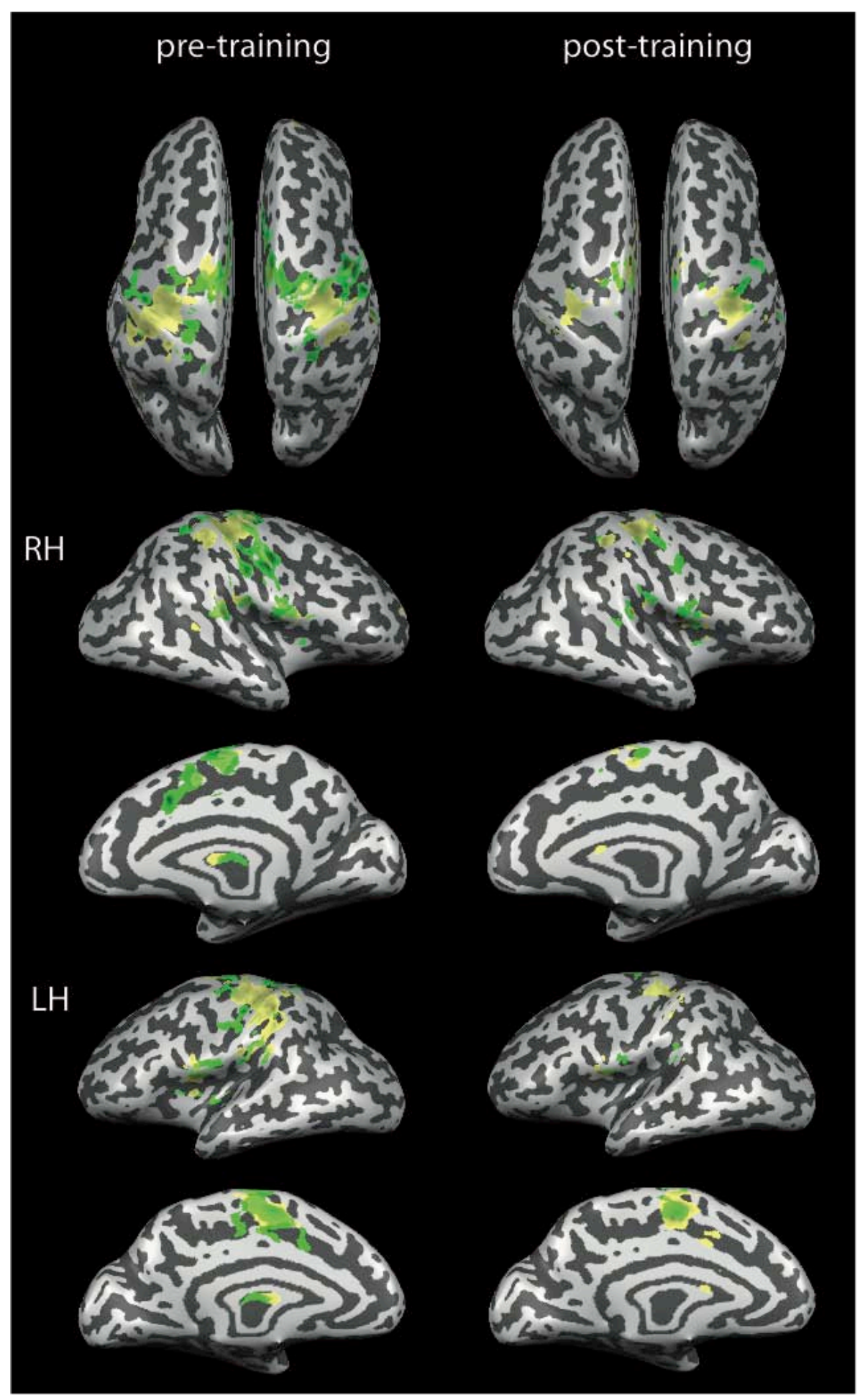

Figure 23) Reduction of motor-related activation cluster size in fMRI through training in SCIsubjects.

Whole brain fMRI activations for all tasks vs. resting state (green=PAM; yellow=FT, group averages $(\mathrm{n}=9)$ ) were calculated using a general linear model with random effects and then projected on the inflated brain surface of a representative subject. Data from the pre-training session is shown on the left and from posttraining on the right. At the top, the superior view of both hemispheres is shown. Below are the lateral and medial views of the right ( $\mathrm{RH})$ and left hemisphere ( $\mathrm{LH}$, in descending order). The threshold was set to $t(8)=4.00$, corresponding to $p<0.00395$. Darker colors indicate stronger $t$-values. Activated areas include the M1, S1, pre-motor cortex, SMA, pre-SMA and hypothalamus. Significant reductions of activation cluster sizes on the M1 through training were detected for the PAM (left hemisphere) and FT (right hemisphere). Recruitment of the medial leg area for PAM activation decreased with training. 


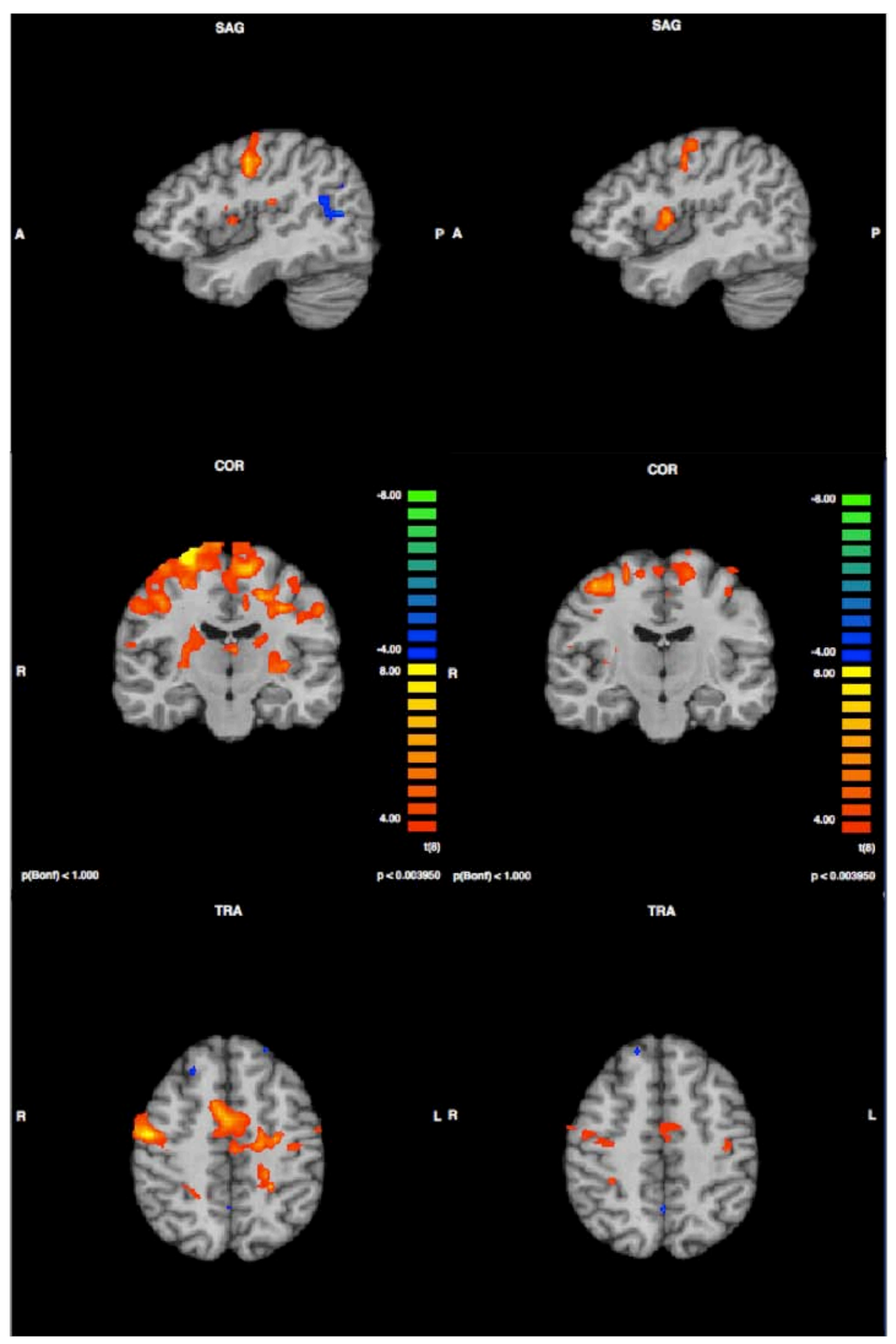

Figure 24) Whole brain group analysis of during PAM activation pre-training (left) and post-training (right).

Sagittal view of the right hemisphere (above), coronal view (middle) and transverse view (below) of mean activations at $\mathrm{x}=42 ; \mathrm{y}=15 ; \mathrm{z}=46$ in Talairach coordinates. A random effect model was applied (PAM vs. rest) at a threshold of $p<0.00395$, corresponding to $t(8)>4.00$. Motor training of the PAM led to reduced activation in the right hemisphere. 


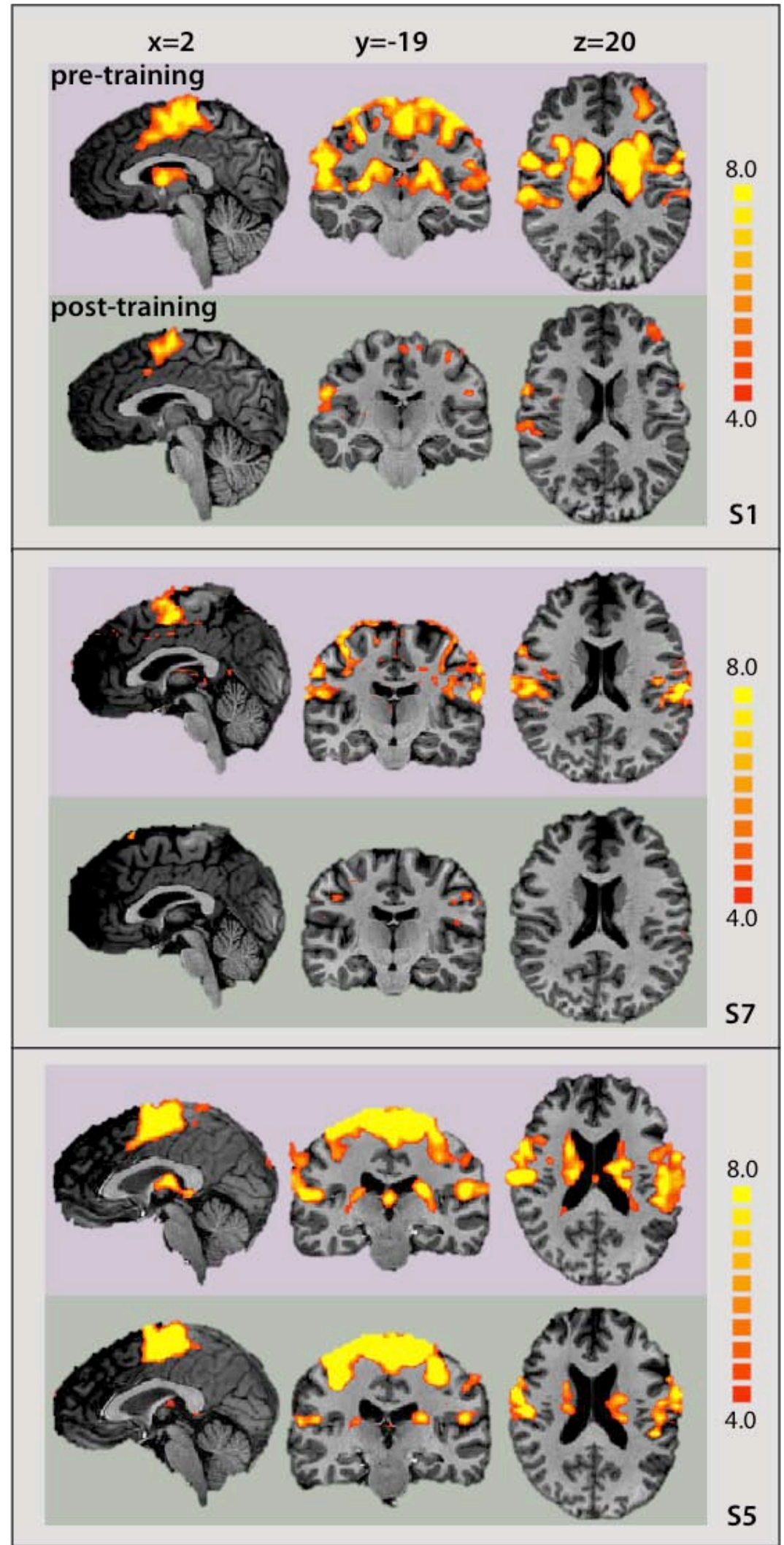

Figure 25) Single subjects' examples of pre- and post-training fMRI activation during PAM activity.

Sagittal view of the right hemisphere (left), coronal view (middle) and transverse view (right) of mean activations during PAM activity at $\mathrm{x}=2, \mathrm{y}=-19, \mathrm{z}=20$ in Talairach coordinates for three SCI subjects. The threshold is set at $p<0.000066$, corresponding to $t(1922)>4$. Subjects 1 and 7 display major activation reduction post-training on the motor cortex as well as in deeper brain structures, e.g. hypothalamus. In subject 5 , the reduction is less pronounced but still present. 


\subsubsection{Comparison of SCI-fMRI to data from able-bodied subjects}

Activation cluster sizes during PAM activity in the first testing were three times larger in our SCI group than in able-bodied subjects (see Table 2; Kruskal-Wallis-test for independent samples; left hemisphere: chi-square=4.688; $p=0.03$; right hemisphere: chisquare=7.293; $p=0.007$ ). This difference was abolished by training (LH: $p=0.85$; RH: $p=0.32$ ). With finger tapping there was no difference before training (all $p>0.05$ ), but able-bodied subjects had larger activation sizes after training in the right hemisphere (RH: chi-square=7.298; $p=0.006$; LH $p=0.089$ ).

Regarding the center of gravity of task-related activation, we found a significant shift of the PAM COG before training in all three dimensions compared to able-bodied subjects (see Table 2 for coordinates and Figure 22; all $p<0.05$ ). This difference persisted after training. The COGs for FT, on the other hand, were remarkably similar between SCI and able-bodied subjects before and after training (all $p>0.05$ ).

\subsubsection{Correlation of neuronal activation and behavioral performance}

We found a direct correlation between motor cortex activation sizes and performance in computer testing. In the pre-training session, those participants with more extensive left hemisphere activation during PAM performed better in unilateral activation than those with less extensive M1 activations ( $r=0.783, p=0.013$, Figure $26 \mathrm{~B}$ ). This changed in the post-training session, where those with less extensive activations had a somewhat more successful unilateral activation $(r=-0.383, p=0.308)$. The correlation was less strong for the right hemisphere, but showed the same directional shift with training (pre-training: $r=0.567$, post-training: $r=-0.417$ ). Accordingly, the better the unilateral activation was post-training, the larger were the cluster size reductions with training in the left hemisphere $(r=-0.717, p=0.030)$. Surprisingly, the pre-training FT cluster size also correlated with better unilateral activation (only right hemisphere, $r=0.700, p=0.036$ ).

We also found a direct correlation between size of pre-training left hemisphere motor cortex activation during PAM movement and post-training performance in the virtual obstacle course (path length: $r=-0.667 ; p=0.05$; completion time: $r=-0.650 ; p=0.058$ ). The correlation was weaker for the right hemisphere (path length: $r=-0.267 ; p=0.488$; completion time: $r=-0.517 ; p=0.154$ ). This means that subjects with more extensive pre- 
training M1 activations in the left hemisphere were faster after training. Also, the cluster size reduction in the left hemisphere (post-training - pre-training) for PAM activation correlated significantly with the virtual obstacle course completion time $(r=-0.733$; $p=0.025$ ). The same correlations were found for right hemisphere M1 activations during FT in the pre-training session (path length: $r=-0.733$; $p=0.025$; completion time: $r=-$ 0.783; $p=0.013$ ).

Regarding real wheelchair tasks, participants with increased PAM activations in the right hemisphere in the pre-training session were faster in the slalom in the final testing session ( $r=-0.67, p=0.05$, Figure $26 \mathrm{C}$ ). Again, this correlation was reversed after training $(r=0.17, p=0.6)$. There was a similar trend for the left hemisphere (pre-training: $r=-0.60$, $p=0.08)$ and a similar reversion post-training $(r=0.23, p=0.5)$. Interestingly, the same was true for the left hemisphere activations during FT imagination in the pre-training session $(r=-0.700, p=0.036)$. Cluster size reduction showed no significant correlations with wheelchair driving. Larger M1 activation sizes for PAM activation pre-training showed a trend to correlate with a faster performance of the real obstacle course in the first session, but only in the left hemisphere $(r=-0.628, p=0.07)$. 


\section{PAM fMRI activation cluster sizes \\ pre-training \\ post-training}

\section{A) Correlation to lesion level}

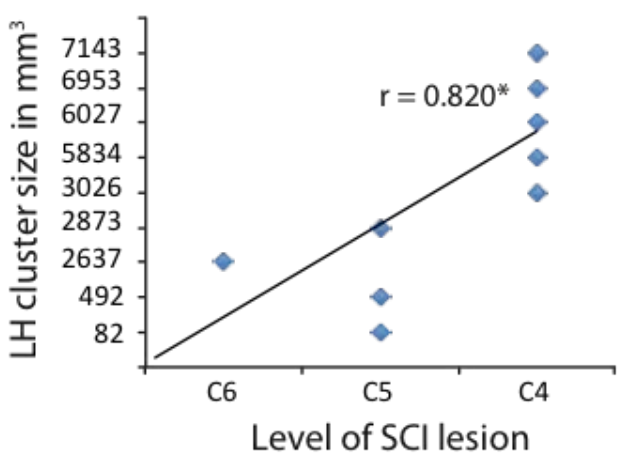

B) Correlation to unilateral activation of PAM

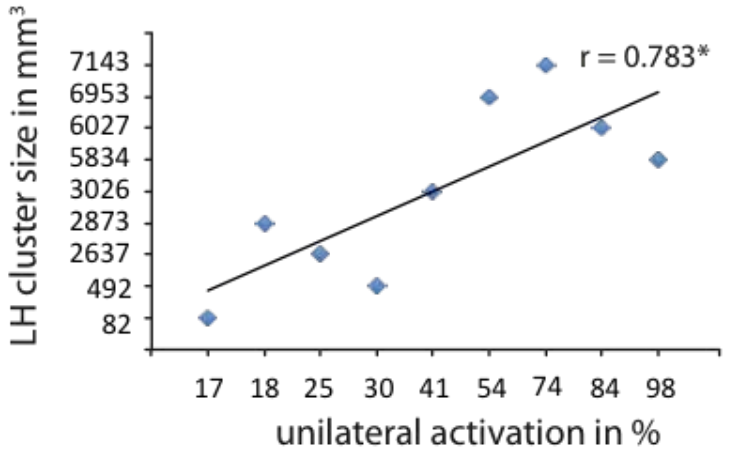

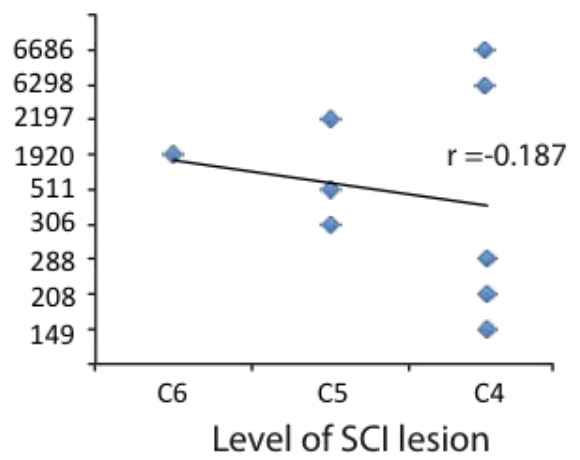

\section{C) Correlation to wheelchair performance - slalom}
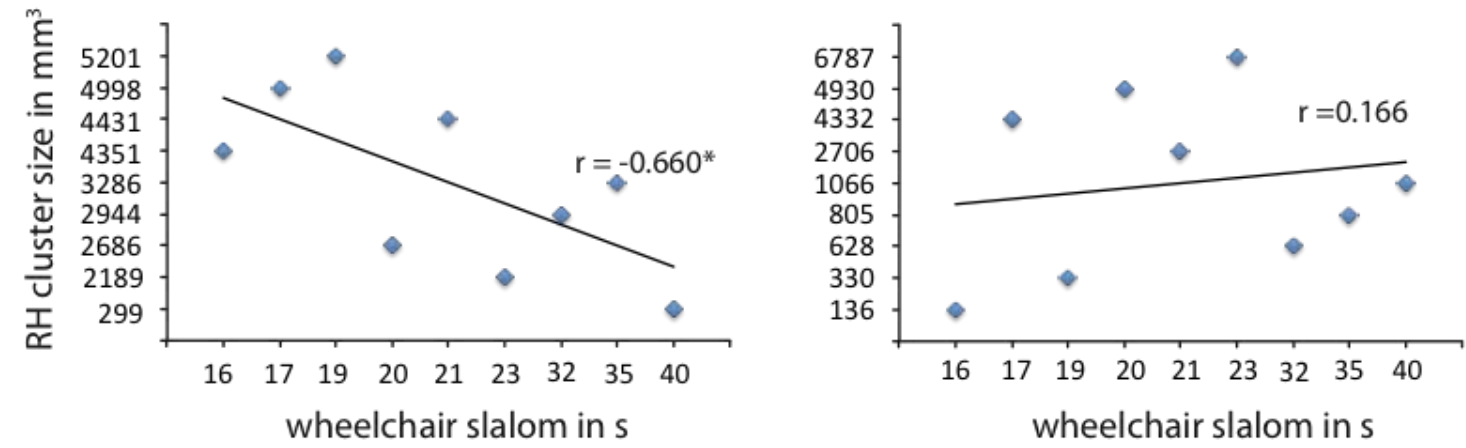

Figure 26) Spearman correlations of fMRI activation cluster sizes during PAM activity pre- and posttraining.

The PAM cluster sizes of the left hemisphere (LH) from the SCI subjects correlated significantly to the level of SCI lesion pre-training (A), i.e. participants with higher lesions had more extended activations on the motor cortex. This correlation was reversed through training, showing a tendency for training to inverse this effect (right side). On a behavioral level, in the pre-training session, those participants with larger LH cluster sizes performed better in unilateral activation (B). Through training, this correlation was turned negative, i.e. smaller cluster sizes tended to go along with improved unilateral activation. Cluster sizes in the right hemisphere in the pre-training session correlated with better wheelchair driving performance (faster slalom completion) posttraining (C). Again, this correlation was reversed through training; post-training cluster sizes did not correlate with better slalom performance. Note that non-parametric spearman correlations were used, which are based on ranks; hence the axes are not continuous. 


\subsubsection{Mapping of muscle representations using TMS}

Coordinates of mean center of gravities (COG) of PAM MEPs in MNI space, and cluster sizes from pre-and post-training sessions are given in Table 2 (see also Figure 27). The coordinates on the $\mathrm{x}$ and $\mathrm{z}$ axes did not change with training $(Z=-0.943, p=0.345 ; Z=-$ 0.631, $p=0.528$ ). However, we found a significant posterior shift on the $y$-axis with training $(Z=-2.023, p=0.043)$.

The effect of training on the size of M1 activation was not significant. However, like in the fMRI data, individuals with higher lesions showed significantly more extended activations in the pre-training session $(r=0.866, p=0.012)$, while this effect was completely abolished post-training $(r=0.0, p=1.0)$. Accordingly, participants with higher lesions showed a trend to greater cluster size reductions with training $(r=-0.722, p=$ $0.67)$.

A direct statistical comparison with the data of the able-bodied subjects was not possible due to the different stimulation settings. Their mean PAM COG was -50, 2 and 51 in MNI space (only left hemisphere, Figure 27), indicating a shift into the coronal direction in our SCI subjects.

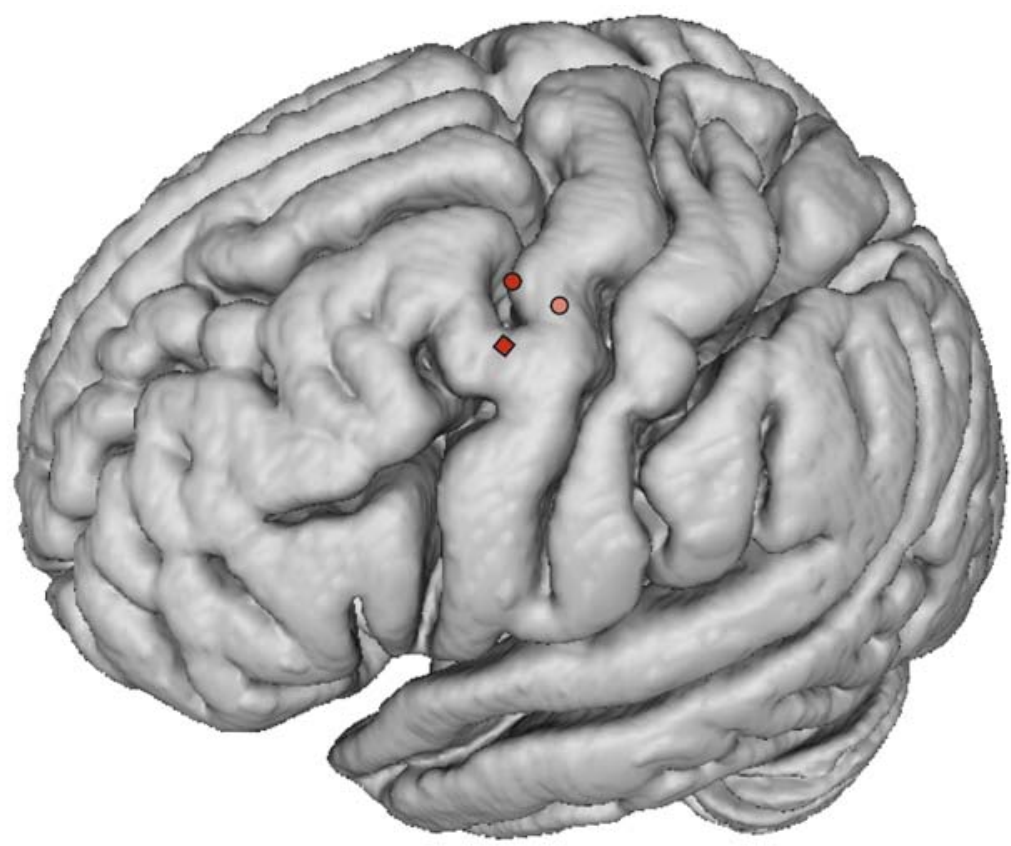

Figure 27) Centers of Gravity (COGs) of TMS motor evoked potentials of the right PAM.

COGs of participants with tetraplegia (circles) and able-bodied subjects previously acquired in our working group (square). Already pre-training (dark red), individuals with tetraplegia had a shift of COGs into medial direction when compared to able-bodied subjects. Training induced a posterior shift (light red). 


\section{Following page:}

Table 2) Talairach coordinates of centers of gravity (COGs) and activation cluster sizes of taskrelated activations in fMRI (in $\mathbf{~ m m}^{3}$ with standard deviations) in the primary motor cortex.

Subjects moved their auricular muscles rhythmically (PAM) or imagined bilateral finger tapping (FT) during fMRI, before training (pre-training) and after 12 days of auricular wheelchair control training (post-training). Significant differences between sessions are marked by *. Activation cluster sizes were significantly reduced during PAM and FT in the left and right hemisphere, respectively. For the same time points, TMS mapping identified mean COGs (in MNI space, with standard deviations) of the left hemisphere cortical area that evoked motor potentials in the right posterior auricular muscle. Training induced a posterior shift of the TMS COG. FMRI data was compared to fMRI data from 13 able-bodied subjects previously acquired in our working group. COGs of the PAM had shifted before training towards the hand area. Clusters size was three times greater in SCI than in able-bodied subjects before training, and this difference was reduced in the post-training session. 


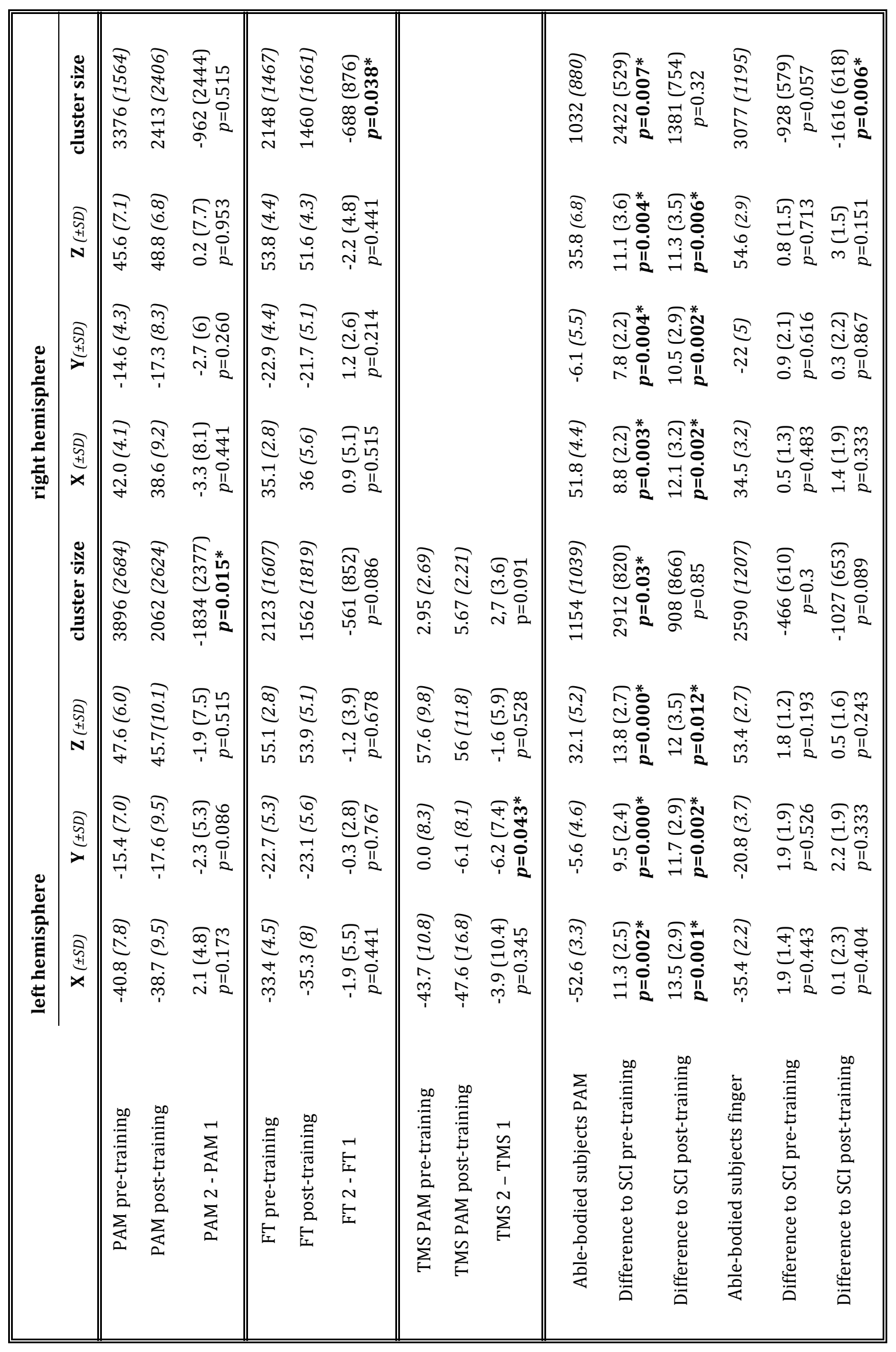




\subsection{Discussion}

For the first time, the functionality of the ACS was shown in individuals with spinal cord injuries. All participants acquired control over their auricular muscles, which they then improved. They were able to improve their performance in complex wheelchair tasks, which demonstrated the feasibility of the ACS as a reliable control system.

Functional MRI data prior to training revealed over-activation of the primary motor cortex during motor tasks in our SCI subjects when compared to data previously acquired from able-bodied subjects. This over-activation correlated with better performance in the ACS. Motor training led to a significant reduction of primary motor cortex activation. After training, less extended M1 activations correlated with better performance. The centers of gravity (COG) of M1 activity during PAM activation had shifted into the direction of the hand area before training. This shift was reinforced by training. The data from these SCI subjects reveals extensive brain reorganization before training and indicate a normalization of brain activity in the course of motor training.

\subsubsection{The ACS as wheelchair control: performance of SCI}

This was the first time that a group of individuals with tetraplegia was trained to use the ACS. They all improved their ability to activate their ear muscles and to distinguish between both sides. Although the system theoretically needs only small differences between the two ears to detect an intention to turn, better unilateral activation allows one to make use of the proportional and continuous features. For example, with a $50 \%$ single-sided activation, the wheelchair turns on the spot. With $70 \%$ activation of the left side and an additional activation $20 \%$ of the right side, the wheelchair turns left with a slight forward motion. Better unilateral activation thus permits a flexible, precise signal generation. The participants significantly improved their ability to activate only one auricular muscle. By the end of the training, they managed an average of $75 \%$ singlesided activation, i.e. without co-activation of the other ear. This final value allows for precise modulation of signal strength while driving an electric wheelchair.

Accordingly, the complex task of steering a real wheelchair improved significantly with training. The continuous and proportional signal generation permits e.g. the online modulation of commands and the direct control over the intended direction. This is an advantage over other control options, such as sip-and-puff or, potentially, brain 
computer interfaces (BCI)(Diez et al., 2013; Kim et al., 2013). Despite the variability in performance, the successful completion of wheelchair tasks shows that participants were able to make use of the flexibility provided by the ACS not only during computer testing, but also during actual wheelchair driving.

The participants not only learned to activate the new muscles but also to attribute a new function to these formerly unused muscles, i.e. that of generating reliable steering signals for their wheelchair. The results are particularly impressive because all but two participants had initially declared themselves unable activate their ear-muscles. Considering the long training time required for control systems such as BCI (Donoghue, 2008) this result is promising.

In a reproduction of the positive evaluations from our previous study (Schmalfuss et al., 2016), the SCI subjects were satisfied with the ACS. According to our own test and the NASA TLX (Hart \& Staveland, 1988), the mental demands for steering a wheelchair after two weeks of training were low, and in the overall evaluation of the system, the participants considered it to be ready for use in daily life.

The positive evaluation together with performance of our participants shows the ACS to be a feasible alternative whenever hands-free control is required. The results of the study showed that the inherent characteristics of the ACS, such as proportional and continuous control, can meet the specific needs of wheelchair users and make it a promising control interface.

\subsubsection{Motor cortex reorganization in chronic SCI: superior PAM control as a result of over-activation?}

In our SCI patients, fMRI activation sizes for motor imagery of FT activation are remarkably similar to those of movement execution by able-bodied subjects. The same relationship has been found earlier, where motor imagery activation size in SCI patients was similar to that of able-bodied subjects executing foot movements (Alkadhi et al., 2005).

In contrast, PAM-related motor cortex activations were three times more extended in SCI subjects than in able-bodied subjects. These larger PAM-related motor cortex activations support studies that have found an enlargement of motor cortex representations in SCI patients (Levy et al., 1990; Lundell, Christensen, et al., 2011; 
Moxon et al., 2014; Turner et al., 2003). Other studies have reported no change(Mattia et al., 2006; Sabbah et al., 2002) or decreased activation (Cramer et al., 2005; HotzBoendermaker et al., 2008). These differences might have been due to a heterogeneity of the studied patients, since those with higher lesion levels seem to have larger motor cortex activation (Bruehlmeier, 1998; Ghosh et al., 2009; Lundell, Christensen, et al., 2011). In accordance with this, we found in the pre-training data a correlation between higher SCI lesion levels and larger fMRI cluster size activation in the left motor cortex during PAM activity. The TMS data confirmed this correlation. Moreover, the same correlation was found for higher SCI lesion levels and larger motor cortex cluster sizes during FT imagination.

The mechanisms of this increased motor-related activation are still incompletely understood (Nardone et al., 2015). Previous studies have suggested that the expansion of motor-related cortex activation is associated to atrophy of the lateral spinal cord (Lundell, Christensen, et al., 2011), reduced afferent inhibition (Ziemann et al., 1998), or could be caused by an overuse of the muscles still available (Sabre et al., 2013). Since PAM had not been overused prior to the study, the latter explanation does not apply to our data. An alternative explanation might be that the disuse of large parts of the motor cortex leads to a reorganization, which enables a recruitment of broader cortical areas for any motor task. The resulting flexibility of the primary motor cortex can also be seen in the recruitment of the de-efferented leg and foot areas of the M1 (medial wall) for PAM activation in a majority of our subjects.

On a functional level, the over-activation of the M1 for motor tasks has so far been seen as a compensational mechanism (Lundell, Christensen, et al., 2011; Turner et al., 2003). The exact function is less clear but it has been proposed that it helps to secure motor output (Di Rienzo et al., 2014; Freund, Rothwell, et al., 2011; Moxon et al., 2014) and might lead to functional gain(Nardone et al., 2013). On the other hand, it might also be responsible for maladaptive changes that can lead to pain and spasticity (Raineteau \& Schwab, 2001) and thus actually be a hindrance to functional recovery (Cramer et al., 2005; Hotz-Boendermaker et al., 2008; Nardone et al., 2015) and impair motor performance (Freund, Weiskopf, et al., 2011). Our data provide the first evidence that the cortical expansion of motor maps in SCI is not necessarily maladaptive, but can actually help produce a superior outcome. In our study, those with a higher lesion level had significantly better scores in unilateral activation and real obstacle course 
performance in the pre-training sessions. Since we have seen that patients with higher lesion levels have larger activations, we believe that the superior performance might also be explained by the provision of additional neuronal resources that accompany a higher lesion. As has been suggested previously, increased activations might thus ensure the capability to respond to motoric requirements and help to respond to new tasks in a flexible manner (Raineteau \& Schwab, 2001). Correlations of more extended pretraining activation sizes and better performance in the post-training session e.g. between PAM and virtual obstacle course or real wheelchair slalom, support the interpretation that additional resources promote the response to new tasks and subsequently also increase the learning effect. When harvested appropriately, e.g. through physiotherapy, the brain reorganization could thus even result as an advantage. Future research should investigate whether with the appropriate exercises, SCI patients would be able to mobilize the additional neurological actuators and achieve better output in other tasks that involve muscles above the lesion level.

\subsubsection{Training reduces motor cortex activity and increases performance}

The initial over-activation was reduced by training during both motor tasks in our subjects. Likewise, the pre-training recruitment of the leg area was virtually abolished in seven of the nine participants after training. It can be ruled out that the decrease was caused by poorer performance, since all participants improved their performance with training. These results suggest that the abnormal over-activations of brain activity commonly found in chronic tetraplegic individuals (Cramer et al., 2005) can actually be normalized by motor training. Whereas before training, patients with higher lesion levels had had greater motor cortex activations during PAM activity, this correlation turned negative after training: higher lesion levels tended to be associated with less expanded motor-related M1 activation than lower lesion levels. The positive correlation of larger TMS cluster sizes with higher lesion levels was likewise abolished by training. The same was true for SMA activations in fMRI, where subjects with higher lesion levels had a significantly greater reduction of activation with training than those with lower lesion levels. In our study, motor training was apparently able to attenuate the influence of SCI lesion levels on motor network activity. 
On a behavioral level, the training-induced decrease in motor cortex activation was accompanied by better performance. Participants with a greater PAM cluster size reduction performed better post-training (e.g. in unilateral activation, virtual obstacle course performance). Remarkably, while in the pre-training session larger cluster sizes had correlated with better performance, this correlation was reversed post-training, for example in unilateral activation and wheelchair slalom. Motor training, thus, seems to change the dynamics of neuronal recruitment during motor tasks in SCI patients. When the motor cortex is engaged with new tasks, training replaces the initial over-activation with increased effectiveness.

In able-bodied subjects, skilled motor training usually leads to an extension of the cortical representation (Elbert \& Flor, 1999; Martin Lotze, Braun, Birbaumer, Anders, \& Cohen, 2003; Perez, Lungholt, Nyborg, \& Nielsen, 2004). However, little is known about the effects of motor training on SCI-specific brain reorganization (Kokotilo et al., 2009). To our knowledge there are only two case studies using TMS (Chisholm et al., 2015; Hoffman \& Field-Fote, 2007) and one series of cases using fMRI (Winchester et al., 2005) indicating that motor training (from $1 \mathrm{~h}$ to 3 weeks) might increase M1 activation in SCI subjects. The results of the TMS studies are not comparable to our fMRI data. The fMRI study, on the other hand, used fMRI tasks that involved impaired muscles below lesion level, leading to only minimal motor cortex activation in the beginning. The same applies to the study by Jurkiewicz et al. who found that functional improvement without training correlated with increased M1 activation during the first year of recovery (Jurkiewicz et al., 2007). The extensive pre-training M1 activation during the above lesion PAM activation in our participants might have led to different neuronal normalization mechanisms, i.e. a reduction in M1 activity.

The specific dynamics of neuronal activity in our study suggests that motor-training effects on cortical organization might be different in individuals with SCI than in ablebodied subjects. In our subjects with tetraplegia, improvement in behavioral performance goes along with a partial decrease of M1 activation and a parallel SMA decrease. A similar neuronal specification, i.e. decrease of neuronal activity associated with improved performance, has been found in the motor cortex of SCI subjects during motor imagery (Di Rienzo et al., 2014; Guillot et al., 2008). The principle of neuronal specification also operates in able-bodied subjects during motor tasks. For example, in professional sports experts recruit smaller motor areas than non-experts for motor 
preparation (Milton, Solodkin, Hluštík, \& Small, 2007). In line with our interpretation, this reduction is viewed as an increase in effectiveness, i.e. that the task can be completed using fewer resources (Poldrack, 2000). An increased understanding of the SCI-specific motor training effect on cortical organization might also be relevant in the context of BCI and help resolve issues with unstable signal classification and exceedingly long training times in individuals with tetraplegia (Kauhanen et al., 2006; Pfurtscheller, Müller, Pfurtscheller, Gerner, \& Rupp, 2003).

However, since SCI subjects did not specifically train motor imagery of FT, the question remains why fMRI activation decreased during the study. Conjunction analysis (shared area between FT and PAM activation) suggests that the peak of shared activation is actually located well inside the hand area. Since both representations are located in the same cortical area, the FT area might have been affected by the training-induced reorganization of the PAM area. Another possibility is that, since PAM activation and motor imagery of FT are both new tasks, the SCI-specific motor learning dynamics of over-activation followed by specification through training apply to both tasks. This interpretation is supported by correlations of FT cortex reduction and better ACS performance, which were similar for PAM-related cortex activity. A single imaging session might possibly have already improved the motor imagery quality and thus reduced the activation size, a correlation that has been shown previously (Alkadhi et al., 2005).

\subsubsection{Shifts of activation before and after training: which motor cortex areas are used?}

The similarities between the COGs of SCI and able-bodied subjects during imagined and executed finger movements are in line with other findings of motor imagery sharing the same neuronal substrates as physical execution (Di Rienzo et al., 2014; Porro et al., 1996; Sabbah et al., 2002). The post-SCI brain reorganization apparently did not affect the original motor program of the de-efferented cortex. In contrast, pre-training PAM COGs had already shifted considerably towards the hand area (Figure 22). This finding is in line with studies that report shifts into the de-efferented limb area in individuals with tetraplegia (Bruehlmeier, 1998; Mikulis et al., 2002) and contradicts the interpretation that these shifts occur predominantly in lower lesion SCI (Kokotilo et al., 2009; M Lotze, 
Laubis-Herrmann, Topka, Erb, \& Grodd, 1999). In addition to extensive brain reorganization, we still found a significant difference between the COGs of FT and PAM in the pre-training session. Training induced another, albeit smaller, PAM COG shift into the hand area, abolishing the pre-training difference.

This means that parts of the unused hand areas are recruited for the new task, while their original motor program remains intact. This "peaceful cohabitation" of different body representations in the same cortical area has been shown previously for phantom limb phenomena (Reilly \& Sirigu, 2008). Preservation of previous motor representations, therefore, does not exclude an involvement in new tasks for deefferented regions (Sabbah et al., 2002).

The shift of PAM COG was more pronounced in the TMS data. As in the fMRI data, SCI subjects showed different locations of PAM COG pre-training than able-bodied subjects. After SCI subjects had trained PAM activation, we found a clear posterior shift. These findings contradict the partial shift into the de-efferented limb area shown by the fMRI data. In general, the posterior shift in the post-SCI brain is interpreted as involving the primary sensory cortex (S1) for motor activities (Nardone et al., 2013). Green et al. argument that the pyramidal axons descending from the S1 might be spared more often from injury due to a more posterior location (Green et al., 1998), thereby achieving better motor outputs. This might also explain the difference between COGs in the fMRI and TMS data, since we did not include the S1 in the fMRI ROI-Analysis. Although all TMS COGs were well within the boundaries of the motor cortex, we cannot rule out the possibility that due to individual anatomical variations some frontal parts of the S1 were also stimulated and thus contributed to the posterior shift. Previous studies have found a similar difference between TMS and fMRI data, arguing that this might be due to a dispersed maximum of current flow, which might not be located directly under the TMS coil (Martin Lotze et al., 2003).

\subsubsection{Limitations of the study}

A limitation of our study is the small sample size, which is a problem in most studies with SCI subjects (Nardone et al., 2015). We also did not evaluate completeness of lesion or the motor score. We believe that these limitations may have contributed to the asymmetry of our results, i.e. that the PAM-related activation reduction following 
training was only significant in the left hemisphere. The pattern of correlations, e.g. that both right hemisphere PAM activation size and higher lesion levels correlated with better slalom performance, or that right hemisphere cluster sizes correlated with better slalom performance pre-training, but not post-training (as found for the left hemisphere in other tasks), suggests that the lateralization might not be functional, but due to experimental factors. It must be pointed out that asymmetry of fMRI results is a common problem in SCI studies (Freund et al., 2013) and that most studies with SCI subjects have trouble detecting consistent effects that apply to all tested subjects (e.g. (Cramer, Orr, Cohen, \& Lacourse, 2007; Green et al., 1999; Sabbah et al., 2002; Shoham, Halgren, Maynard, \& Normann, 2001; Turner et al., 2003)). This might be due to the heterogeneity of SCI patients (Kokotilo et al., 2009) who often differ in multiple important factors at the same time.

The scope of this study was to investigate the effects of motor training in SCI patients, but in order to infer a specificity of our results, the same training should be conducted with control subjects. However, motor training studies suggest different results in individuals in whom the somatotopic organization of the motor cortex is still intact, e.g. no over-activation, less activation reduction, or even increased activation through training.

\subsection{Conclusion}

In this study, we have provided further evidence that the ACS is indeed a viable control option for subjects with tetraplegia. All participants learned to activate their PAM and to generate precise wheelchair steering signals during the twelve days of training. Our results suggest that the brain reorganization in SCI subjects is two-fold: compared to able-bodied subjects, we found extensive brain reorganization with activation shifts into the de-efferented hand area and increased activation cluster sizes. Correlations of greater activations clusters with superior ACS performance suggest that the increased availability of neuronal structures can optimize motoric output. Motor training, on the other hand, caused a reduction in activation sizes during motor tasks, similar to neuronal specification (improved effectiveness) in other motor paradigms. This reduction might indicate a normalization of SCI brain activity through training. Whether the focus of neuronal activation is task-specific because a new muscle is used, or 
corresponds to general skill-acquisition should be addressed in future experiments. We provide the first evidence that the post-SCI brain reorganization can be specifically targeted with training interventions. These results could have important consequences for the adequate physiotherapeutic care of SCI subjects, e.g. choosing appropriate tasks in order to make use of additional neuronal potential, and possibly ameliorate negative consequences of the brain reorganization. 


\section{Conclusion of this thesis}

\subsection{Summary of the results}

In the presented studies we have tested the newly developed ACS in different applications for control of assistive devices. We have also investigated the effects of motor training during ACS use on the brain reorganization of subjects with tetraplegia.

In the first study, we trained ten able-bodied subjects and two individuals with tetraplegia for four consecutive days with visual feedback and computer games in their ability to voluntarily activate their bilateral PAM. All subjects showed significant improvements in different parameters of muscle control, e.g. contraction rate, length of contraction and importantly, unilateral activation. Significantly, there was no difference between those subjects who could already activate their PAM before the study and those who could not. By the fifth training day all subjects were able to generate reliable control signals, steering an electric wheelchair using only the ACS. Furthermore, subjects with tetraplegia were able to complete a complex obstacle course. With this study, we proved the feasibility of the ACS for wheelchair control, thereby making use of the inherent advantages of this control, e.g. not interfering with other activities and proportional and continuous signal generation.

In the second study, the ACS was combined with an established control mode in order to provide simultaneous control over two DOF for a prosthetic hand. 10 able-bodied subjects and one subject with transradial amputation used the hACS and two other clinically established control modes in two performance tests with a prosthetic hand. Even without training, performance results were superior with the hACS in terms of precision and speed. This result was replicated by the more experienced prosthesis user. The hACS could therefore resolve some of the problems with the established prosthesis controls and might be a viable alternative to other proposed academic control options.

In the third study, the clinical application of the ACS to wheelchair control was tested with nine subjects with tetraplegia. Twelve days of training resulted in significantly improved performance in the control over the auricular muscles (e.g. unilateral activation) and in the control of an electric wheelchair (e.g. slalom, obstacle course). fMRI and TMS data revealed extensive brain reorganization when compared to data from the able-bodied subjects before training, with shifts of activation maxima and 
increased activation cluster sizes on the primary motor cortex when moving their auricular muscles. Importantly, increased activation sizes were correlated with better performance, suggesting an increased flexibility due to additional neuronal resources. ACS-related motor training on the other hand reduced this excessive activation and caused a neuronal specification for the task. These results provide a first insight into SCI-specific activity-dependent neuronal plasticity and could have important consequences for the therapeutic care of individuals with SCI.

\subsection{Final conclusion and future directions}

With this series of studies we have shown that the ACS is indeed a viable control alternative for assistive devices. The ACS was able to respond to the different demands associated with both wheelchair and prosthesis control. Different inherent features of the ACS resulted in advantageous control properties. For example, the continuous signal transmission allowed for fluent, s-formed curves in wheelchair control and for flowing, natural hand movements in prosthesis control. The proportional control improved speed in prosthesis control, because unlike in e.g. SL, the signal amplitude could be used exclusively for speed of movement. In wheelchair driving, speed of the wheelchair was determined by strength of contraction. Subjects started with slower wheelchair driving and increased speed during the course of training in e.g. the obstacle course, showing that they were able to modulate speed according to their demands whilst making use of the proportional feature of this control interface. User evaluations of the ACS qualities were very positive and suggested that it was ready for daily use.

Significantly we attributed a new function to a previously unused muscle, making use of an unexploited potential especially relevant for individuals with high level tetraplegia. Conflicting results had previously been reported over this vestigial muscle and whether it could be voluntarily activated. With the presented studies, we provide evidence that the voluntary activation of the PAM can be learned and trained. Besides simple on-off activation, our subjects achieved complex activation patterns (e.g. unilateral activation, modulation of activation strength). We were able to show that despite being small, the auricular muscle can generate command signals precise enough for those in a 
wheelchair to complete a complex obstacle course, whilst also making fewer mistakes in prosthesis control.

Subjects learned control over this new muscle with relatively little training using simple visual feedback. In the first study we have seen that those subjects who were already able to activate their PAM before training, had an advantage on unilateral activation that persisted after training. From our following studies, we cannot definitively state whether this difference would be abolished through longer training or not. However, although e.g. better unilateral activation produces clearer signals and potentially widens the variety of possible signals, our system can also work with a minimal difference between both sides to detect and intended turn. If subjects can learn to produce a difference between both sides, they are thus able to use the ACS. The results from our studies suggest that also those subjects who are naïve to the task readily learn unilateral activation with little feedback training and are able to use the ACS for complex wheelchair driving. However, for a wider application of the ACS an epidemiological study is needed, in order to understand if the ability to learn voluntary PAM activation can be generalized to a larger population.

Co-contraction of the PAM during facial movements is a potential influence on signal quality. We did not systematically investigate this in our studies, because in our experience this co-activation was minimal. These co-activations can occur to a certain extent during e.g. broad smiling. However, from our experience the influence on signal quality was negligible, because in a normal environment these extreme facial movements occur rarely and can be avoided by the user if necessary, without limiting other activities.

We used semi-invasive fine-wire electrodes for EMG deduction of the PAM in order to improve signal quality. For a wider application, an implanted version would greatly reduce set up time and increase independence of the user.

The results presented here indicate that the ACS has a wide field of potential application. In theory it could also be used in a non-clinical context, whenever hands-free control is needed. Our subjects with tetraplegia suggested an integrated application for e.g. environmental control, in order to achieve tasks of daily living like switching on the lights or a television. We have provided evidence that the ACS is suitable for signal generation, thus alternative application fields are only a question of programming. Since 
the ACS has been developed for those individuals who do not have other viable control options, we only aimed to show that the wheelchair driving performance is satisfying. Although wheelchair-driving results were very positive, future work should include a direct comparison to other control options, such as the sip-and-puff, for benchmarking. For a clinical implementation of the ACS for wheelchair control, a potential combination with a shared control approach could be advantageous. We did not investigate safety aspects of the ACS, which could be considerably improved by e.g. external sensors or automatic obstacle avoidance.

In the last part of this thesis, first insights into a SCI-specific motor cortex organization were provided. This is the first time that data from a larger SCI sample has been reported that suggests that post-SCI brain reorganization is not static, but can be targeted with training. In order to improve rehabilitation for individuals with SCI, future research should address whether these effects can be generalized to other motor training paradigms. We have seen in our SCI subjects that improvement correlates with decrease in neuronal activity. Future studies should address the specificity of this neuronal specification with a similar motor training in able-bodied subjects. Difficulties in finding SCI subjects who are willing to affront the logistic challenges of study participation (e.g. transport, hospitalization) make small sample sizes a general problem in SCI studies. In order to make clear inferences on the nature of post-SCI brain reorganization, bigger sample sizes are necessary.

In conclusion, in this thesis a new control system for assistive devices was presented, which due to its inherent features could solve some of the problems with clinically established and academic control systems. We were able to show that the human auricular muscles can be voluntarily controlled and can generate precise control signals, hence attributing a new function to these unused muscles. The hybrid auricular-forearm prosthesis control can improve speed and precision of a prosthetic hand. We have shown that the ACS is a suitable alternative for individuals with tetraplegia, answering the need for precise, direct and fast wheelchair control that does not interfere with other activities. Importantly, this thesis has provided data for a better understanding of the SCI-specific brain reorganization. Cortical over-activation might lead to increased flexibility and apparently motor training is associated with neuronal specification, possibly inducing a normalization of brain activity. 


\section{Appendix}

\section{I) Questionnaire about ability to activate the PAM}

1) Wie gut kannst du deine Ohrmuskulatur aktivieren?

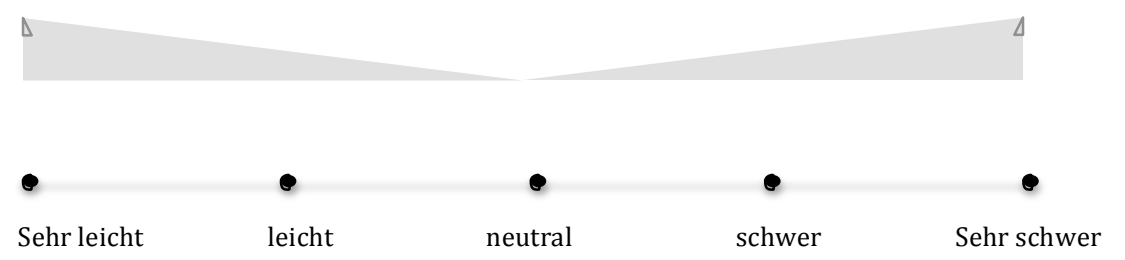

2) Wie anstrengend ist es für dich, deine Ohrmuskulatur zu aktivieren?

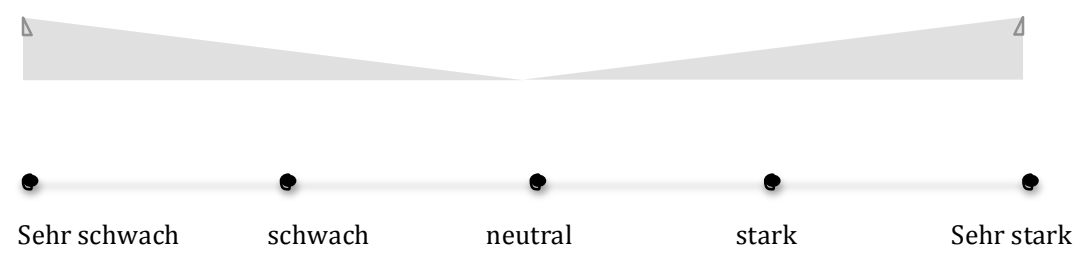

3) Wie sehr musst du dich auf deine Ohrmuskulatur konzentrieren, um einen Effekt zu erzielen?

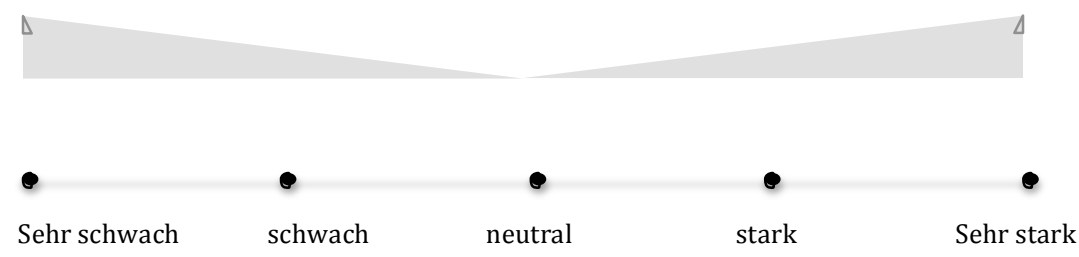

4) Wie leicht fällt es dir, die Ohrmuskulatur nur auf einer Seite zu aktivieren?

a) Links:

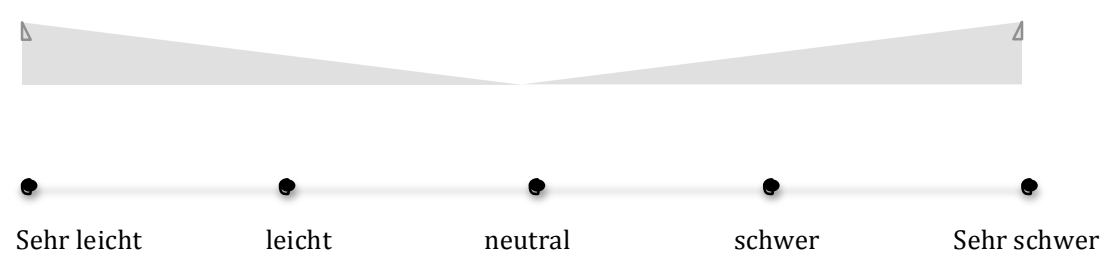

b) Rechts:

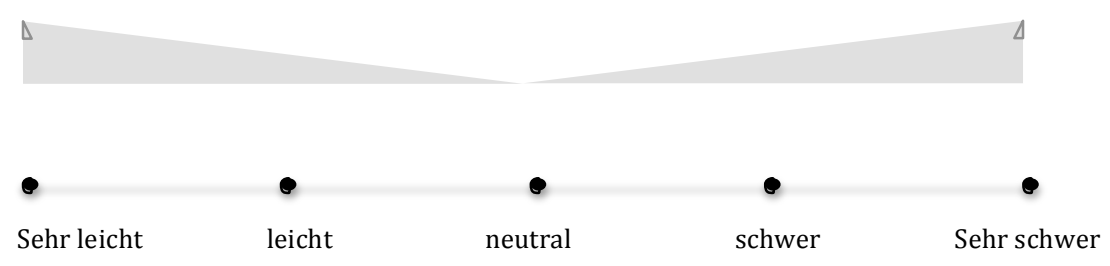


5) Wie sehr musst du andere Muskeln mit anspannen, um die Ohrmuskulatur zu aktivieren?

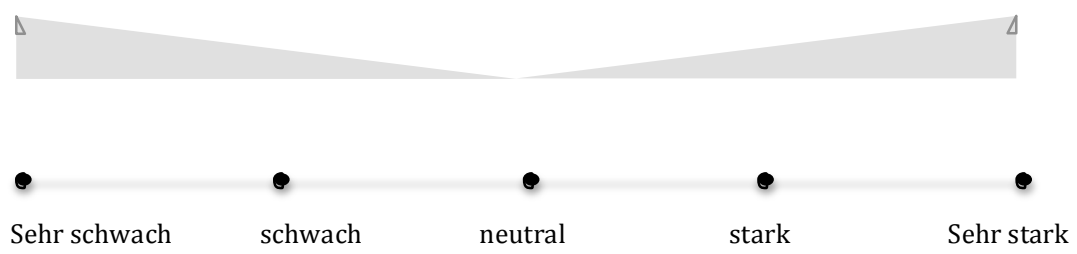




\section{II) Evaluation of the ACS}

1) Glauben Sie, es ist möglich mit diesem System einen Rollstuhl im Alltag zu nutzen?

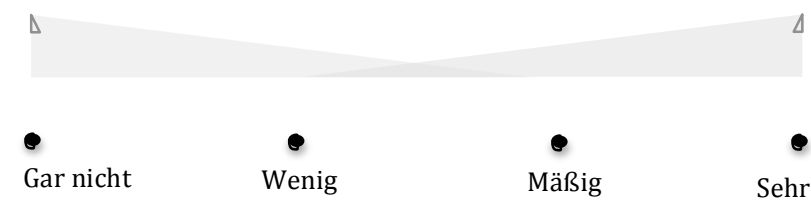

2) Wie schwierig war es für Sie, den Rollstuhl präzise zu steuern?

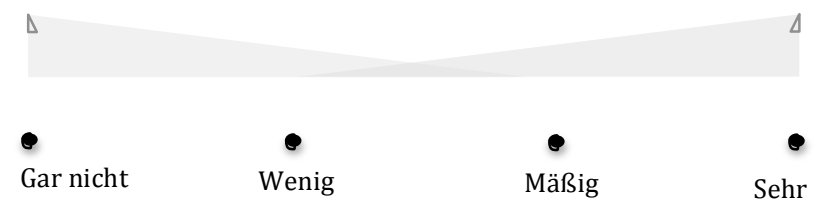

3) Würden Sie mit diesem System ihren Rollstuhl steuern wollen?

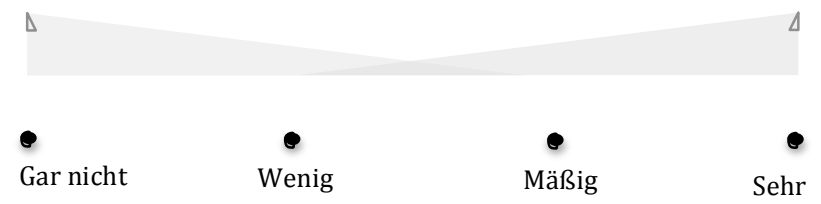

4) Wie gut funktionieren...

a) ... enge Kurven

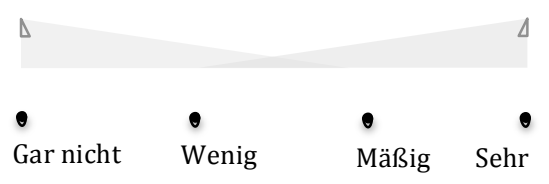

c) ... geradeaus fahren

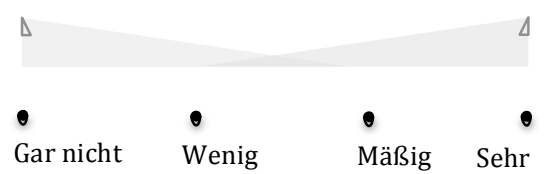

e) ... Genauigkeit

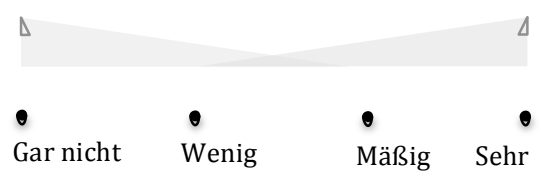

b) ... weite Bögen

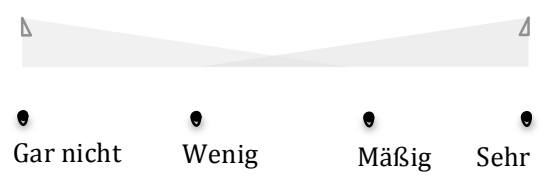

d) ... Reaktionsschnelle

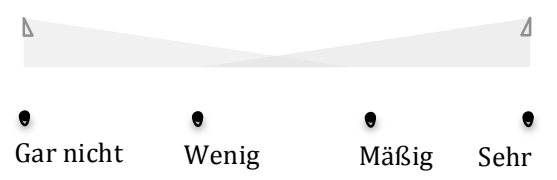

f) ... Schnelligkeit

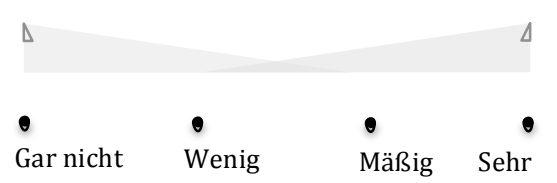


5) Wieviel Training glauben Sie wäre nötig um den Rollstuhl sicher zu beherrschen?

6) Was sind besondere Vorteile der Ohrsteuerung?

7) Was sind mögliche Schwierigkeiten, was könnte man verbessern?

8) Was wären - für Sie persönlich - andere sinnvolle Einsatzmöglichkeiten der Steuerung?

9) Hatten Sie bisher eine andere Rollstuhlsteuerung? Welche?

Wenn Ja,
a) wie sind Sie mit dieser zurecht gekommen?
b) Was sind Vorteile im Vergleich zur Ohrsteuerung?
c) Was sind Nachteile im Vergleich zur Ohrsteuerung?

10) Haben Sie sonst noch Kommentare, Verbesserungsvorschläge oder Anregungen für uns? 


\section{III) Supplementary material}

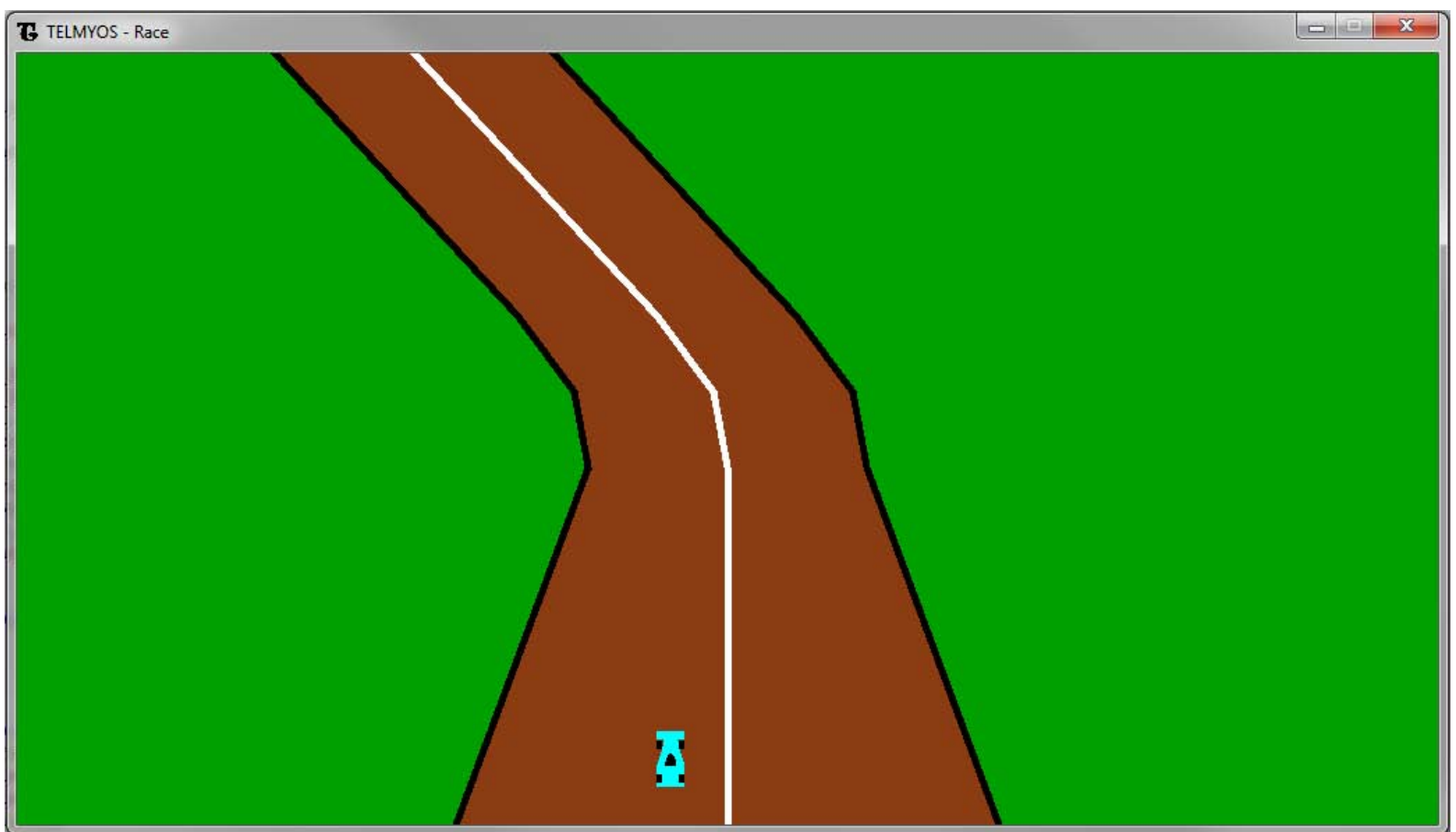

\section{Supp. Fig. 1) Training PAM activation - car race.}

In car race, the virtual car had to be kept on the moving track with left and right movements (left and right contractions). The percentage of the time that the virtual car was correctly driving on the path was reported as a feedback. Four levels with increasing difficulty (more narrow turns and paths) were included.

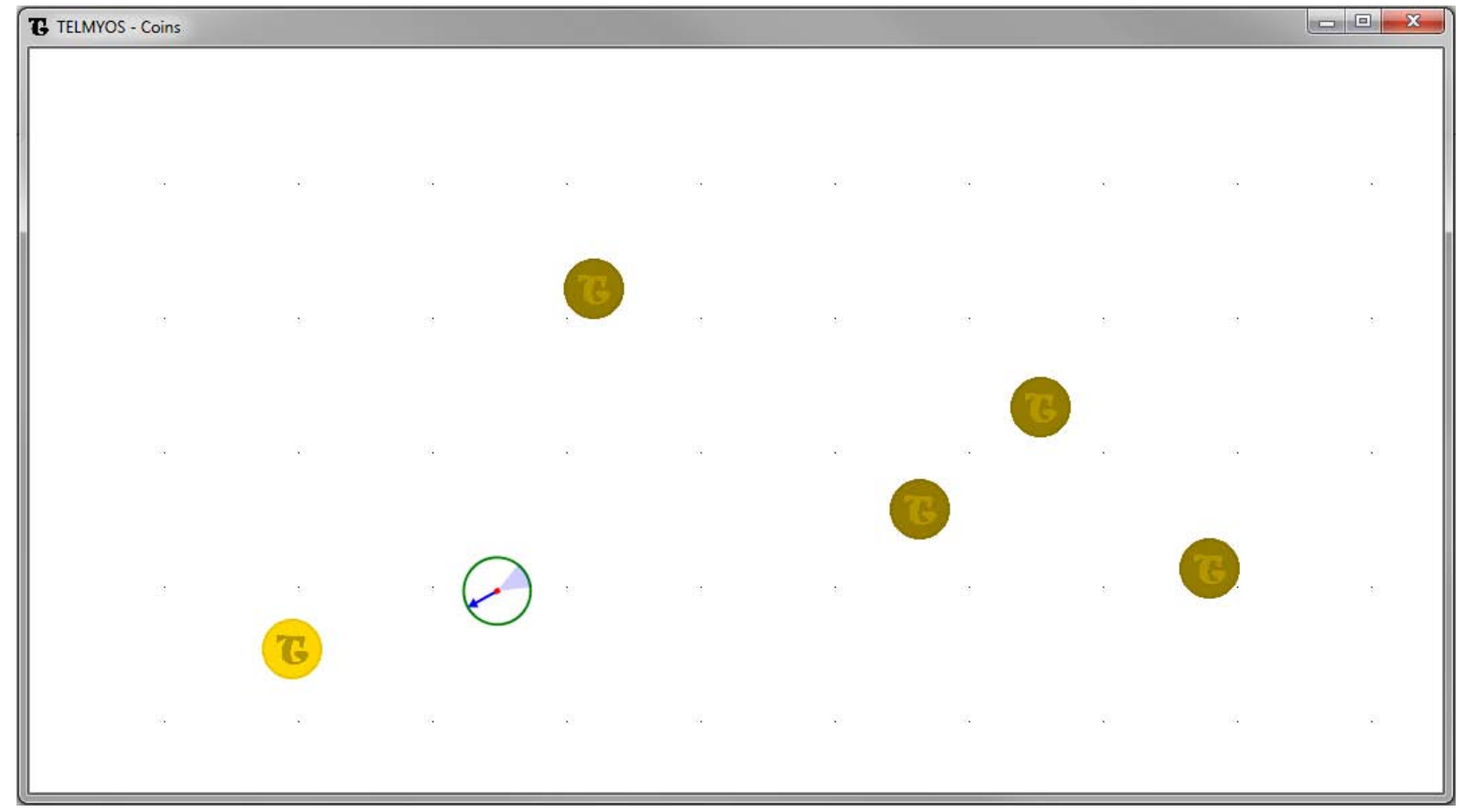

\section{Supp. Fig. 2) Training PAM activation - collecting coins.}

In coins collecting, subjects steered a virtual vehicle with the same steering signals as in real wheelchair driving. Whenever a coin popped up, the subject had to drive over it to collect it, then another one popped up until ten were collected. 


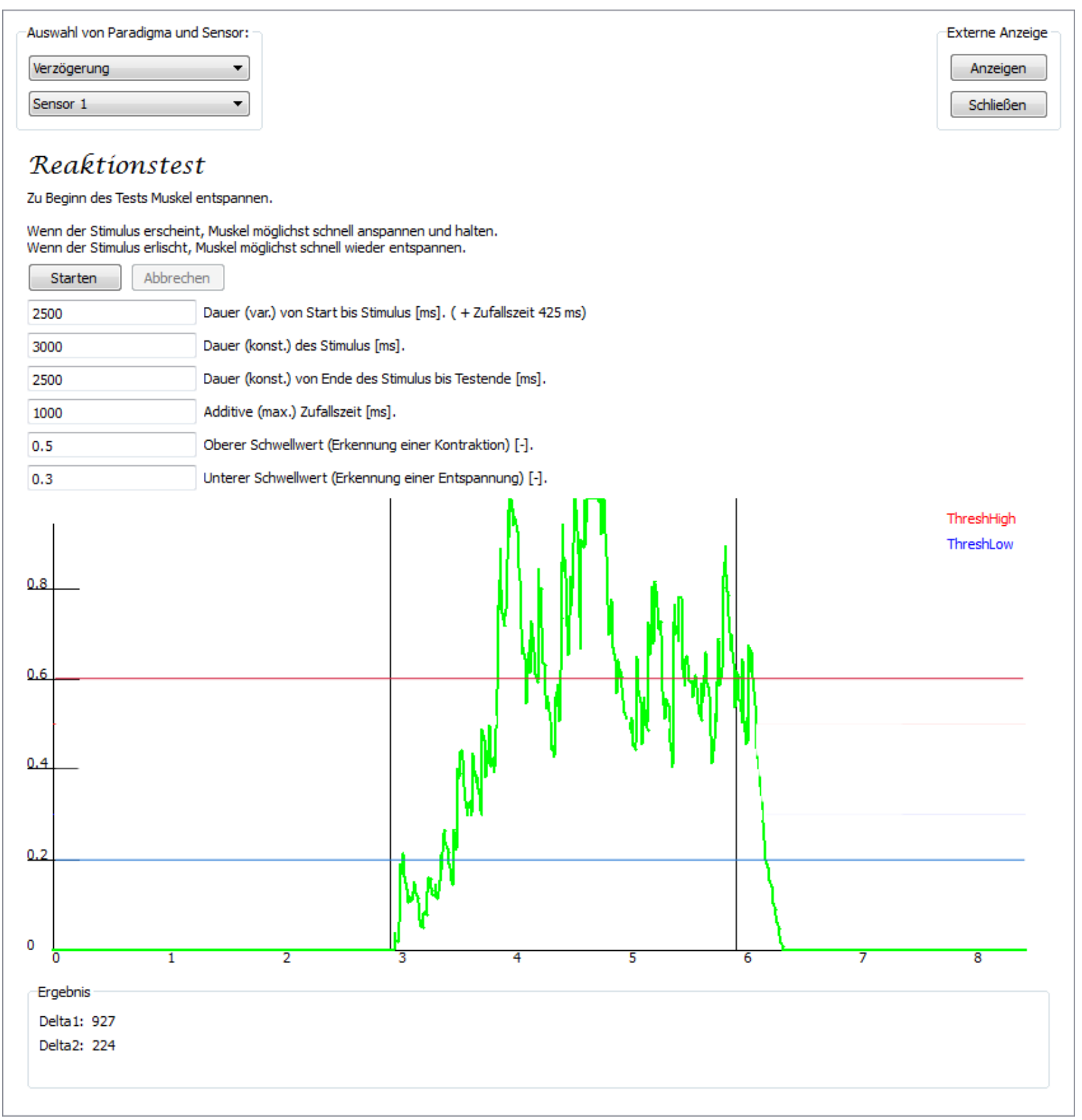

\section{Supp. Fig. 3) Test for reaction times (RT) of contraction and relaxation.}

Screenshot of the muscle activity (green line) during reaction time test. Subjects saw a green square on the screen and were told to contract the PAM as soon as the square turned red (indicated with (A)), hold the contraction and relax the muscle as soon as the square turned green again (indicated with (B)). The time between appearance of the red square and the successful contraction (passing the threshold of 0.6, (C)) was recorded as RT for contraction and the time between appearance of the green square and a correct relaxation (activation below the threshold of $20 \%$, (D)) as RT for relaxation. 


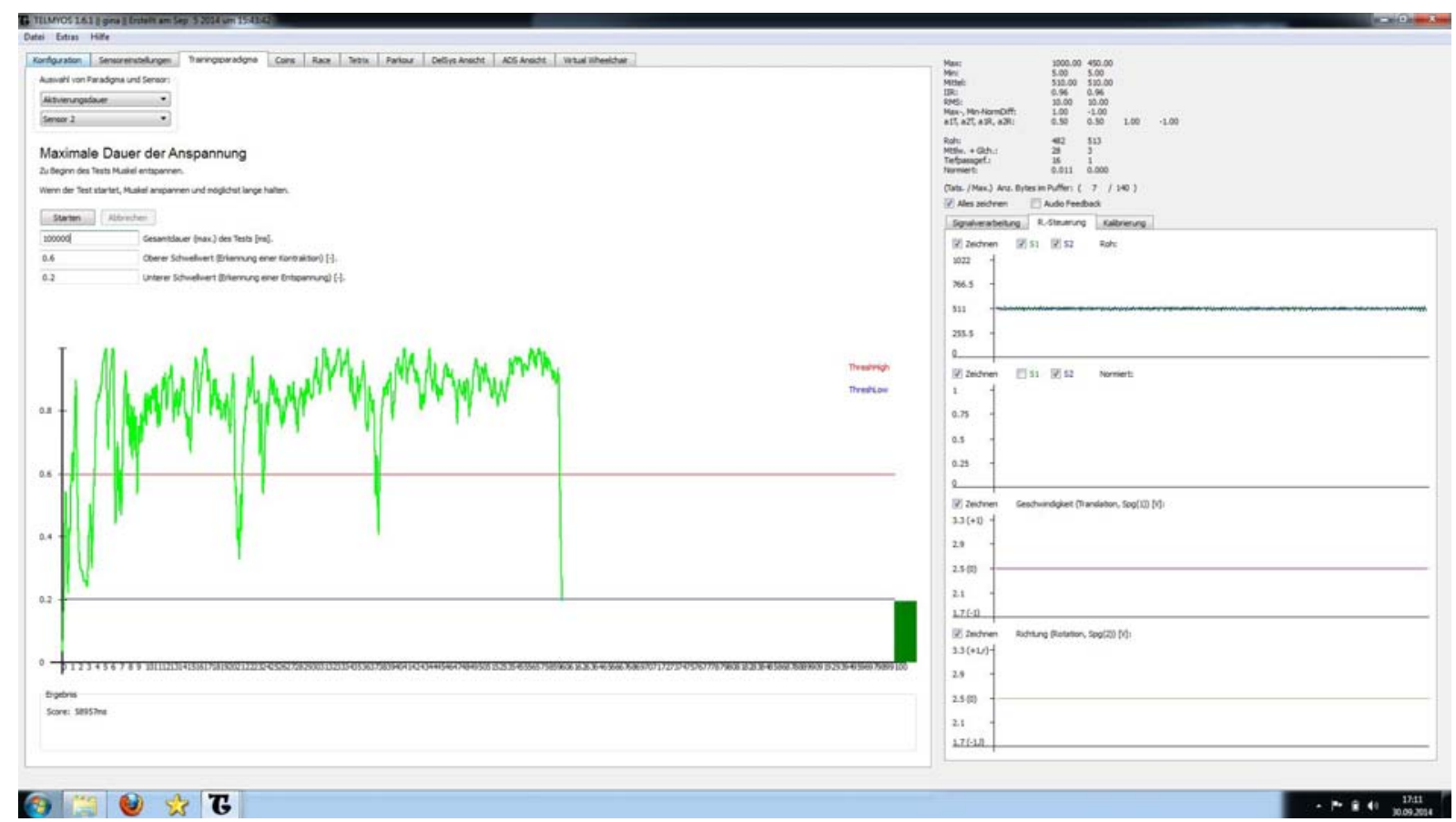

\section{Supp. Fig. 4) Test for maximum contraction duration.}

Screenshot of the software that converts the raw EMG signals (A) into normalized signals (B) and control signals ((C), translational signal, (D), rotational signal). Subjects were told to hold a full contraction as long as possible (E). Counting started when the activation passed the upper threshold of $60 \%$ (red line) and stopped when the EMG activity went below the relaxation threshold of $20 \%$ (blue line), i.e. when the contraction failed. This subject held the contraction for $59957 \mathrm{~ms}$, i.e. roughly $60 \mathrm{~s}$.

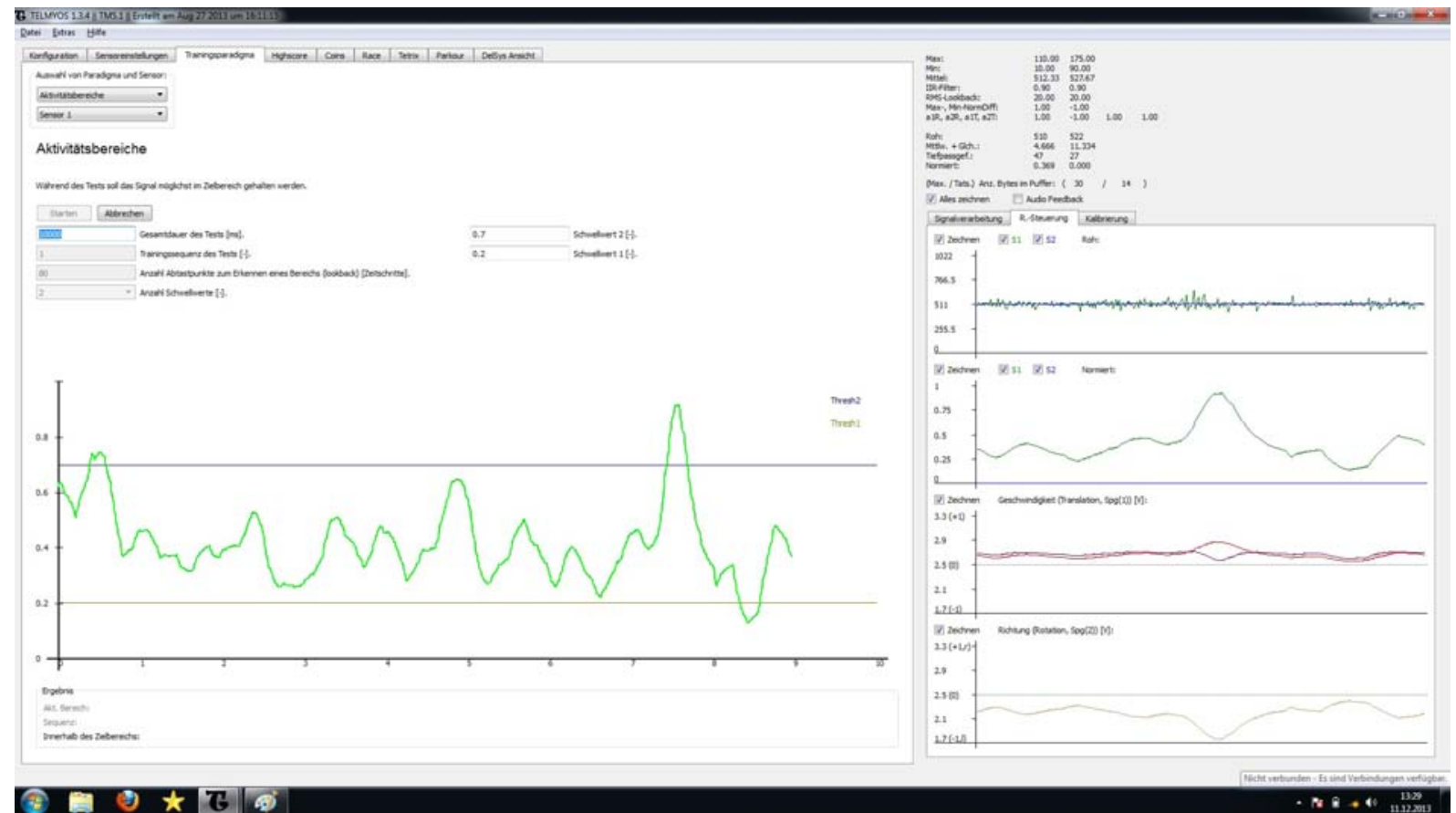

\section{Supp. Fig. 5) Test for graded PAM activity.}

Screenshot of the software that converts the raw EMG signals (A) into normalized signals (B) and control signals ((C), translational signal, (D), rotational signal). Subjects were told to hold the EMG activity between the two thresholds (E), i.e. between 0.2 and 0.7 (20\% and $70 \%$ of the normalized activity). This subject spent $92 \%$ of the time correctly activating its PAM between $20 \%$ and $70 \%$ (score $92 \%$ ), meaning that the subject can successfully grade the muscle activity to a medium activation. 

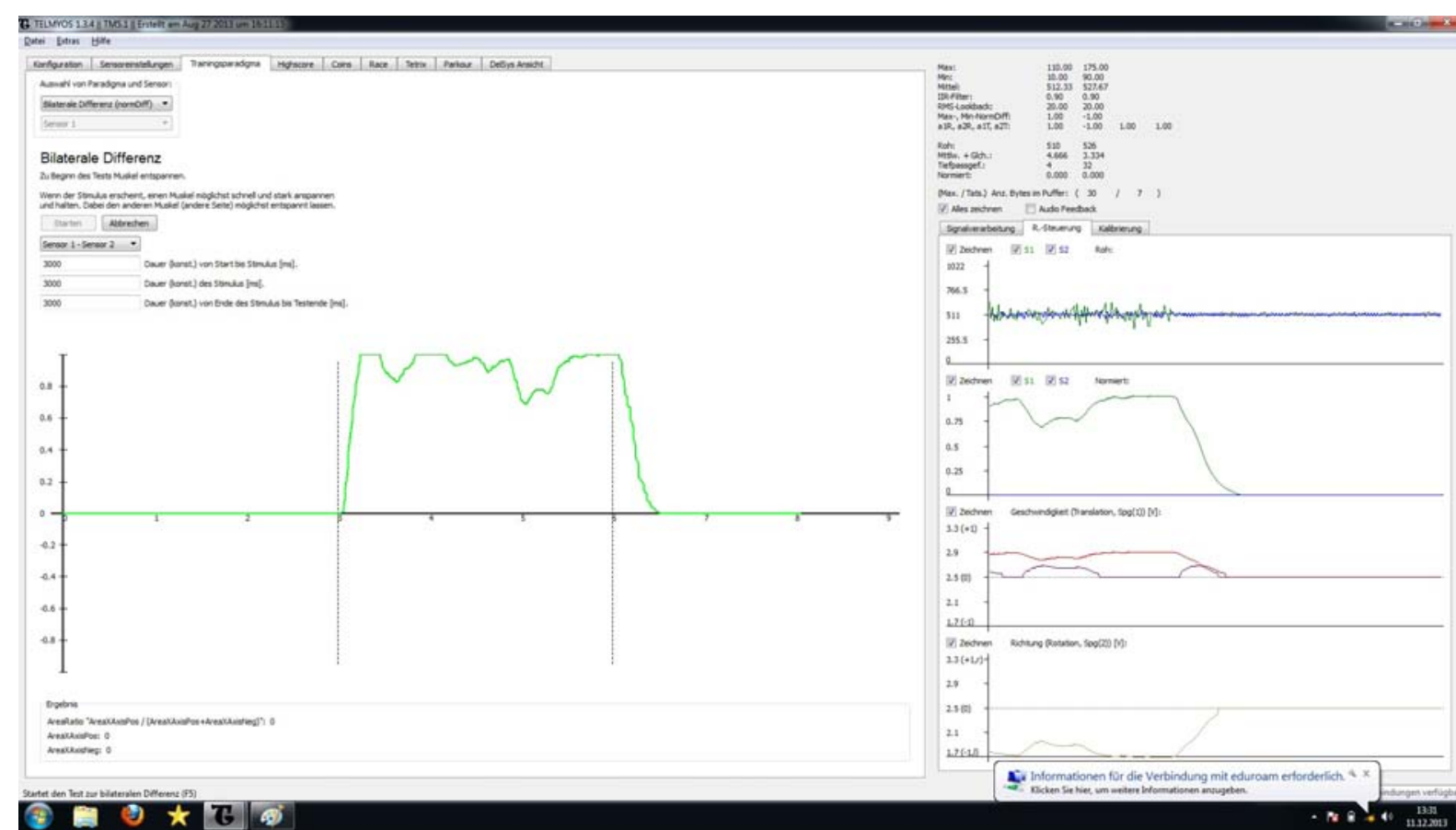

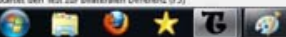

\section{Supp. Fig. 6) Test for lateralized activation.}

Screenshot of the software that converts the raw EMG signals (A) into normalized signals (B) and control signals ((C), translational signal, (D), rotational signal). Subjects were instructed to activate only one side selectively for $3 \mathrm{~s}$. The correct activation in this task was defined as the average individual strength of the correct PAM contraction in percent (e.g. 0.3 of maximum $=30 \%$ ). For the final score, the strength of the unintended contraction of the contralateral PAM is subtracted from the correct activation. This subject managed a final score of $87 \%$ (E), since there was no unintended activation (see (B), only one side is active) and almost full activation over three seconds was achieved. 

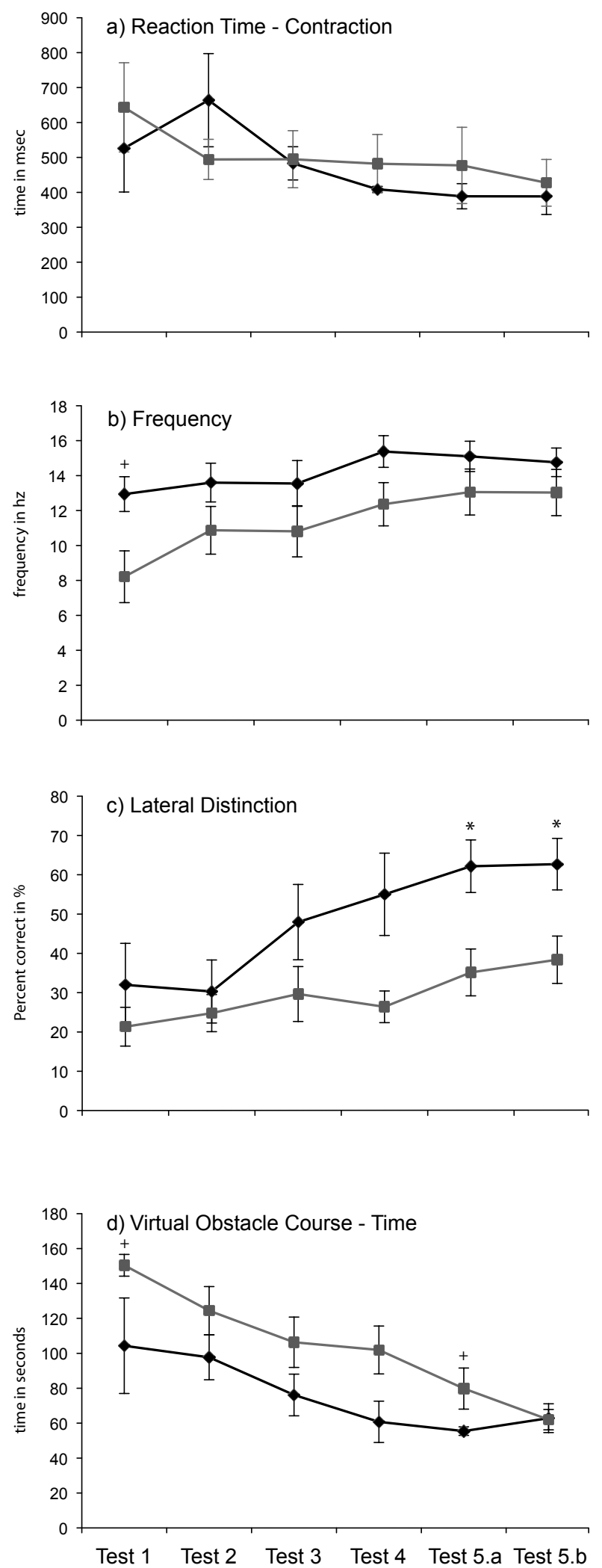

Supp. Fig. 7) Training effects for subgroups of ear-wigglers and non ear-wigglers.

Mean results with standard error for groups of ear-wigglers (green line, $n=5$ ) and non ear-wigglers (blue line, $n=5$ ) in PAM performance criteria: reaction time of contraction (A), frequency of contraction (B), lateralized activation (C) and speed in the virtual obstacle course (D). Significant differences between groups in session 1 and session $5 \mathrm{a}$ and session $5 \mathrm{~b}$ as tested with the independent groups t-tests are marked $+=p<0.05$ or $*=p<0.016$ (Bonferroni corrected alpha level). 


\begin{tabular}{|c|c|c|c|c|c|c|c|c|c|c|}
\hline Criteria & \multicolumn{3}{|c|}{$\begin{array}{l}\text { RM ANOVA } \\
\text { (Tests 1-5 b) }\end{array}$} & $\begin{array}{l}\text { Test } 1 \\
M \pm S D\end{array}$ & $\begin{array}{l}\text { Test } 5 \text { a } \\
M \pm S D\end{array}$ & \multicolumn{2}{|c|}{ vs. Test 1} & $\begin{array}{l}\text { Test } 5 \mathrm{~b} \\
M \pm S D\end{array}$ & \multicolumn{2}{|c|}{ vs. Test 1} \\
\hline RT of contraction & 2.992 & $0.020^{*}$ & .249 & $\begin{array}{l}584.65 \mathrm{~ms} \\
\pm 273.67\end{array}$ & $\begin{array}{l}433.04 \mathrm{~ms} \\
\pm 177.66\end{array}$ & --- & $0.017^{*}$ & $\begin{array}{l}407.88 \mathrm{~ms} \\
\pm 127.79\end{array}$ & -- & $0.007 *$ \\
\hline $\mathrm{RT}$ of relaxation & 4.606 & $0.002^{*}$ & .339 & $\begin{array}{l}691.92 \mathrm{~ms} \\
\pm 316.2\end{array}$ & $\begin{array}{l}524.02 \mathrm{~ms} \\
\pm 236.25\end{array}$ & -- & $0.005^{*}$ & $\begin{array}{l}462.46 \mathrm{~ms} \\
\pm 127.53\end{array}$ & --- & $0.005^{*}$ \\
\hline Contraction rate & 1.08 & $0.000^{*}$ & .545 & $\begin{array}{l}1.06 \mathrm{~Hz} \\
\pm 0.37\end{array}$ & $\begin{array}{l}1.41 \mathrm{~Hz} \\
\pm 0.26\end{array}$ & -4.56 & $0.001 *$ & $\begin{array}{l}1.39 \mathrm{~Hz} \\
\pm 0.25\end{array}$ & -3.85 & $0.004^{*}$ \\
\hline Graded activation & 3.882 & $0.005^{*}$ & .301 & $\begin{array}{l}63.44 \% \\
\pm 12.9 \\
\end{array}$ & $\begin{array}{l}75.11 \% \\
\pm 8.37\end{array}$ & -2.23 & 0.053 & $\begin{array}{l}80.63 \% \\
\pm 6.97\end{array}$ & -4.1 & $0.003^{*}$ \\
\hline Contraction duration & 6.767 & $0.000^{*}$ & .429 & $\begin{array}{l}50.25 \mathrm{~S} \\
\pm 34.28 \\
\end{array}$ & $\begin{array}{l}64.84 \mathrm{~s} \\
\pm 30.41 \\
\end{array}$ & -2.6 & 0.029 & $\begin{array}{l}70.55 \quad s \\
\pm 33.58 \\
\end{array}$ & -3.24 & $0.010^{*}$ \\
\hline Lateralized activation & 8.340 & $0.000^{*}$ & .481 & $\begin{array}{l}26.65 \% \\
\pm 18.3\end{array}$ & $\begin{array}{l}48.66 \% \\
\pm 19.53 \% \\
\end{array}$ & -5.44 & $0.000^{*}$ & $\begin{array}{l}50.54 \% \\
\pm 18.45\end{array}$ & -4.7 & $0.001 *$ \\
\hline \multicolumn{11}{|l|}{$\begin{array}{l}\text { Virtual obstacle } \\
\text { course }\end{array}$} \\
\hline Path length & 5.612 & $0.001 *$ & .412 & $\begin{array}{l}4035.9 \mathrm{px} \\
\pm 989.1\end{array}$ & $\begin{array}{l}3118.6 \mathrm{px} \\
\pm 369.4\end{array}$ & --- & $0.005^{*}$ & $\begin{array}{l}2996.9 p x \\
\pm 272.8\end{array}$ & --- & $0.012 *$ \\
\hline Speed & 6.147 & $0.000^{*}$ & .435 & $\begin{array}{l}127.39 \mathrm{~s} \\
\pm 48.38\end{array}$ & $\begin{array}{l}67.62 \quad \mathrm{~s} \\
\pm 22.08\end{array}$ & -- & $0.007^{*}$ & $\begin{array}{l}62.49 \mathrm{~s} \\
\pm 14.89\end{array}$ & -- & $0.021 *$ \\
\hline
\end{tabular}

Supp. Table 1) Summary of results and statistics of the PAM performance criteria in the first study.

Data from ten able-bodied subjects. Results of the repeated measures ANOVAs with the factor "time" for each criterion (F-values, p-values and effect size Eta2). Mean (M) and standard deviation (SD) in sessions $1,5 \mathrm{a}$ and $5 \mathrm{~b}$. Abbreviation px means pixels. T-values $(\mathrm{t})$ for the differences session 1 vs. session $5 \mathrm{a}$ and session 1 vs. session $5 b$ are displayed when data was normally distributed (Shapiro-Wilk test), if not, the non-parametric Wilcoxon Rank test was used. Significant $\mathrm{p}$-values are marked ${ }^{*}=\mathrm{p}<0.025$ (Bonferroni corrected alpha level). 


\section{Bibliography}

Abbott, A. (2006). Neuroprosthetics: In search of the sixth sense. Nature, 442(7099), 125-127. https://doi.org/10.1038/442125a

Alkadhi, H., Brugger, P., Boendermaker, S. H., Crelier, G., Curt, A., Hepp-Reymond, M. C., \& Kollias, S. S. (2005). What disconnection tells about motor imagery: Evidence from paraplegic patients. Cereb. Cortex, 15(2), 131-140. https://doi.org/10.1093/cercor/bhh116

Amsuess, S., Goebel, P., Graimann, B., \& Farina, D. (2014). Extending mode switching to multiple degrees of freedom in hand prosthesis control is not efficient. Conf Proc IEEE Eng Med Biol Soc, 2014, 658-661. https://doi.org/10.1109/EMBC.2014.6943677

Arshak, K., Buckley, D., \& Kaneswaran, K. (2006). Review of assistive devices for electric powered wheelchairs navigation. ITB J., 7(1). Retrieved from http://biomed.eeng.may.ie/ dbuckley/ITB_Journal_May_2006.pdf\#page=13

Asghari Oskoei, M., \& Hu, H. (2007). Myoelectric control systems-A survey. Biomed. Signal Process. Control, 2(4), 275-294. https://doi.org/10.1016/j.bspc.2007.07.009

Atkins, D. J., Heard, D. C. Y., \& Donovan, W. H. (1996). Epidemiologic overview of individuals with upper-limb loss and their reported research priorities. J Prosthet Orthot, 8. https://doi.org/10.1097/00008526-199600810-00003

Atzori, M., \& Muller, H. (2015). Control Capabilities of Myoelectric Robotic Prostheses by Hand Amputees: A Scientific Research and Market Overview. Front Syst Neurosci, 9, 162. https://doi.org/10.3389/fnsys.2015.00162

Barea, R., Boquete, L., Mazo, M., \& Lopez, E. (2002). System for assisted mobility using eye movements based on electrooculography. IEEE Trans Neural Syst Rehabil Eng, 10(4), 209-218. https://doi.org/10.1109/TNSRE.2002.806829

Bartschat, A., Rupp, R., Liebetanz, D., Meincke, J., Klinker, F., Hewitt, M., \& Reischl, M. (2012). A prototyping environment for evaluation of man-machine interfaces based on electromyographic activity. Biomed. Eng. / Biomed. Tech. https://doi.org/10.1515/bmt-2012-4243

Behrman, A. L., Bowden, M. G., \& Nair, P. M. (2006). Neuroplasticity After Spinal Cord Injury and Training: An Emerging Paradigm Shift in Rehabilitation and Walking Recovery. Phys. Ther., 86(10), 1406-1425. https://doi.org/10.2522/ptj.20050212

Belter, J. T., Segil, J. L., Dollar, A. M., \& Weir, R. F. (2013). Mechanical design and performance specifications of anthropomorphic prosthetic hands: a review. J. Rehabil. Res. Dev., 50(5), 599-618.

Berzin, F., \& Fortinguerra, C. R. H. (1993). EMG study of the anterior, superior and posterior auricular muscles in man. Ann. Anat., 175(2), 195-197. https://doi.org/10.1016/S0940-9602(11)80182-2

Biddiss, E., \& Chau, T. (2007). Upper limb prosthesis use and abandonment: a survey of the last 25 years. Prosthet Orthot Int, 31(3), 236-257. https://doi.org/10.1080/03093640600994581

Birbaumer, N., Ghanayim, N., Hinterberger, T., Iversen, I., Kotchoubey, B., Kübler, A., ... 
Flor, H. (1999). A spelling device for the paralysed. Nature, 398(6725), 297-298. https://doi.org/10.1038/18581

Bland, J. M., \& Altman, D. G. (1995). Multiple significance tests: the Bonferroni method, 310(6973), 170. Retrieved from http://www.ncbi.nlm.nih.gov/pubmed/7833759

Bruehlmeier, M. (1998). How does the human brain deal with a spinal cord injury? Eur. J. Neurosci., 10(September), 3918-3922. Retrieved from http://dx.doi.org/10.1046/j.1460-9568.1998.00454.x

Burke, D. A., Linden, R. D., Zhang, Y. P., Maiste, A. C., \& Shields, C. B. (2001). Incidence rates and populations at risk for spinal cord injury: A regional study. Spinal Cord, 39(5), 274-278. https://doi.org/10.1038/sj.sc.3101158

Carrozza, M. C., Persichetti, A., Laschi, C., Vecchi, F., Lazzarini, R., Vacalebri, P., \& Dario, P. (2007). A wearable Biomechatronic interface for controlling robots with voluntary foot movements, 12(1), 1-11. https://doi.org/10.1109/TMECH.2006.886250

Chainay, H., Krainik, A., Tanguy, M.-L., Gerardin, E., Le Bihan, D., Phane, S., \& Ricy, L. (2004). Foot, face and hand representation in the human supplementary motor $\begin{array}{lll}\text { arear } & \text { Neuroreport, 15(5), }\end{array}$ https://doi.org/10.1097/01.wnr.0000121902.83438.32

Chaves, E. S., Boninger, M. L., Cooper, R., Fitzgerald, S. G., Gray, D. B., \& Cooper, R. A. (2004). Assessing the influence of wheelchair technology on perception of participation in spinal cord injury. Arch. Phys. Med. Rehabil., 85(11), 1854-1858. https://doi.org/10.1016/j.apmr.2004.03.033

Chisholm, A. E., Peters, S., Borich, M. R., Boyd, L. A., \& Lam, T. (2015). Short-term cortical plasticity associated with feedback-error learning after locomotor training in a patient with incomplete spinal cord injury. Phys Ther, 95(2), 257-266. https://doi.org/10.2522/ptj.20130522

Cipriani, C., Controzzi, M., \& Carrozza, M. C. (2011). The SmartHand transradial prosthesis. J Neuroeng Rehabil, 8, 29. https://doi.org/10.1186/1743-0003-8-29

Clement, R. G. E., Bugler, K. E., \& Oliver, C. W. (2011). Bionic prosthetic hands: A review of present technology and future aspirations, 9(6), 336-340. https://doi.org/10.1016/j.surge.2011.06.001

Craig, A., Tran, Y., \& Middleton, J. (2009). Psychological morbidity and spinal cord injury: a systematic review. Spinal Cord, 47(2), 108-114. https://doi.org/10.1038/sc.2008.115

Cramer, S. C., Lastra, L., Lacourse, M. G., \& Cohen, M. J. (2005). Brain motor system function after chronic, complete spinal cord injury, 128(12), 2941-2950. https://doi.org/10.1093/brain/awh648

Cramer, S. C., Orr, E. L. R., Cohen, M. J., \& Lacourse, M. G. (2007). Effects of motor imagery training after chronic, complete spinal cord injury. Exp. Brain Res., 177(2), 233-242. https://doi.org/10.1007/s00221-006-0662-9

Curt, A., Bruehlmeier, M., Leenders, K. L., Roelcke, U., \& Dietz, V. (2002). Differential effect of spinal cord injury and functional impairment on human brain activation. $J$. Neurotrauma, 19(1), 43-51. https://doi.org/10.1089/089771502753460222

Darwin, C. (1859). On the origin of species by means of natural selection, or the 
preservation of favoured races in the struggle for life. London: John Murray.

Dayan, E., \& Cohen, L. G. (2011). Neuroplasticity subserving motor skill learning. Neuron, 72(3), 443-454. https://doi.org/10.1016/j.neuron.2011.10.008

Di Rienzo, F., Guillot, A., Mateo, S., Daligault, S., Delpuech, C., Rode, G., \& Collet, C. (2014). Neuroplasticity of imagined wrist actions after spinal cord injury: a pilot study. Exp. Brain Res., 233(1), 291-302. https://doi.org/10.1007/s00221-014-4114-7

Diez, P. F., Torres Muller, S. M., Mut, V. A., Laciar, E., Avila, E., Bastos-Filho, T. F., ... Sarcinelli-Filho, M. (2013). Commanding a robotic wheelchair with a highfrequency steady-state visual evoked potential based brain-computer interface. Med Eng Phys, 35(8), 1155-1164. https://doi.org/10.1016/j.medengphy.2012.12.005

Donoghue, J. P. (2008). Bridging the brain to the world: a perspective on neural interface systems. Neuron, 60(3), 511-521. https://doi.org/10.1016/j.neuron.2008.10.037

Dosen, S., Cipriani, C., Kostic, M., Controzzi, M., Carrozza, M. C., \& Popovic, D. B. (2010). Cognitive vision system for control of dexterous prosthetic hands: Experimental evaluation. J. Neuroeng. Rehabil., 7. https://doi.org/Artn 4210.1186/1743-0003-742

Dunlop, S. A. (2008). Activity-dependent plasticity: implications for recovery after spinal $\begin{array}{llll}\text { cord injury. Trends } & \text { N1(8), }\end{array}$ https://doi.org/10.1016/j.tins.2008.05.004

Edgerton, V. R., Tillakaratne, N. J. K., Bigbee, A. J., de Leon, R. D., \& Roy, R. R. (2004). Plasticity of the Spinal Neural Circuitry After Injury. Annu. Rev. Neurosci., 27(1), 145-167. https://doi.org/10.1146/annurev.neuro.27.070203.144308

Elbert, T., \& Flor, H. (1999). Magnetoencephalographic investigations of cortical reorganization in humans. Funct. Neurosci. Evoked Potentials Magn. Fields (EEG Suppl. 49), 284-291.

Elbert, T., Pantev, C., Wienbruch, C., Rockstroh, B., \& Taub, E. (1995). Increased cortical representation of the fingers of the left hand in string players. Science (80-. )., 270(1995), 305-307. https://doi.org/10.1126/science.270.5234.305

Engdahl, S. M., Christie, B. P., Kelly, B., Davis, A., Chestek, C. A., \& Gates, D. H. (2015). Surveying the interest of individuals with upper limb loss in novel prosthetic control techniques. J. Neuroeng. Rehabil., $12(1), \quad 53$. https://doi.org/10.1186/s12984-015-0044-2

Englehart, K., \& Hudgins, B. (2003). A robust, real-time control scheme for multifunction myoelectric control. IEEE Trans Biomed Eng, 50(7), 848-854. https://doi.org/10.1109/TBME.2003.813539

Englehart, K., Hudgins, B., \& Parker, P. (2001). Multifunction control of prostheses using the myoelectric signal. Intell. Syst. Technol. Rehabil. Eng., (MAY).

Esquenazi, A. (2004). Amputation rehabilitation and prosthetic restoration. From surgery to community reintegration. Disabil. Rehabil., 26(14-15), 831-836. https://doi.org/10.1080/09638280410001708850

Farina, D., Jiang, N., Rehbaum, H., Holobar, A., Graimann, B., Dietl, H., \& Aszmann, O. C. (2014). The extraction of neural information from the surface EMG for the control 
of upper-limb prostheses: emerging avenues and challenges. IEEE Trans Neural Syst Rehabil Eng, 22(4), 797-809. https://doi.org/10.1109/TNSRE.2014.2305111

Fehr, L., Langbein, W. E., \& Skaar, S. B. (2000). Adequacy of power wheelchair control interfaces for persons with severe disabilities: a clinical survey. J. Rehabil. Res. Dev., 37(3), 353-60. Retrieved from http://www.ncbi.nlm.nih.gov/pubmed/10917267

Foldes, S. T., \& Taylor, D. M. (2010). Discreet discrete commands for assistive and neuroprosthetic devices. IEEE Trans Neural Syst Rehabil Eng, 18(3), 236-244. https://doi.org/10.1109/TNSRE.2009.2033428

Fougner, A., Scheme, E., Chan, A. D. C., Englehart, K., \& Stavdahl, Ø. (2011). Resolving the Limb Position Effect in Myoelectric Pattern Recognition. IEEE Trans. Neural Syst. Rehabil. Eng., 19(6), 644-651. https://doi.org/10.1109/TNSRE.2011.2163529

Freund, P., Curt, A., Friston, K., \& Thompson, A. (2013). Tracking Changes following Spinal Cord Injury. Neurosci., 19(2), 116-128. https://doi.org/10.1177/1073858412449192

Freund, P., Rothwell, J., Craggs, M., Thompson, A. J., \& Bestmann, S. (2011). Corticomotor representation to a human forearm muscle changes following cervical spinal cord injury. Eur. J. Neurosci., 34(11), 1839-1846. https://doi.org/10.1111/j.14609568.2011.07895.x

Freund, P., Weiskopf, N., Ward, N. S., Hutton, C., Gall, A., Ciccarelli, O., ... Thompson, A. J. (2011). Disability, atrophy and cortical reorganization following spinal cord injury, 134(6), 1610-1622. https://doi.org/10.1093/brain/awr093

Ghosh, A., Sydekum, E., Haiss, F., Peduzzi, S., Zörner, B., Schneider, R., ... Schwab, M. E. (2009). Functional and anatomical reorganization of the sensory-motor cortex after incomplete spinal cord injury in adult rats. J. Neurosci., 29(39), 12210-12219. https://doi.org/10.1523/JNEUROSCI.1828-09.2009

Grafton, S. T. (2010). Unlocking communication with the nose. Proc Natl Acad Sci U S A, 107(32), 13979-13980. https://doi.org/10.1073/pnas.1009925107

Gray, H. (1918). Anatomy of the Human Body (18th ed.). Philadelphia: Lea \& Febiger. Retrieved from http://www.bartleby.com/br/107.html

Green, J. B., Sora, E., Bialy, Y., Ricamato, A., \& Thatcher, R. W. (1998). Cortical sensorimotor reorganization after spinal cord injury: an electroencephalographic study. Neurology, 50(4), 1115-1121. https://doi.org/10.1212/WNL.50.4.1115

Green, J. B., Sora, E., Bialy, Y., Ricamato, a, \& Thatcher, R. W. (1999). Cortical motor reorganization after paraplegia: an EEG study. Neurology, 53(4), 736-743. https://doi.org/10.1212/WNL.53.4.736

Guillot, A., Collet, C., Nguyen, V. A., Malouin, F., Richards, C., \& Doyon, J. (2008). Functional neuroanatomical networks associated with expertise in motor imagery. Neuroimage, $41(4)$ 1471-1483. https://doi.org/10.1016/j.neuroimage.2008.03.042

Hahne, J., Dahne, S., Hwang, H.-J., Muller, K.-R., \& Parra, L. (2015). Concurrent Adaptation of Human and Machine Improves Simultaneous and Proportional Myoelectric Control. IEEE Trans. Neural Syst. Rehabil. Eng., 19(6), 644-651. https://doi.org/10.1109/TNSRE.2015.2401134 
Hahne, J. M. M., Graimann, B., \& Muller, K. R. R. (2012). Spatial filtering for robust myoelectric control. IEEE Trans Biomed Eng, 59(5), 1436-1443. https://doi.org/10.1109/TBME.2012.2188799

Hakonen, M., Piitulainen, H., \& Visala, A. (2015). Current state of digital signal processing in myoelectric interfaces and related applications. Biomed. Signal Process. Control, 18, 334-359. https://doi.org/10.1016/j.bspc.2015.02.009

Han, J.-S., Zenn Bien, Z., Kim, D.-J., Lee, H.-E., \& Kim, J.-S. (2003). Human-machine interface for wheelchair control with EMG and its evaluation. Proc. Annu. Int. Conf. IEEE Eng. Med. Biol. Soc. EMBS, 2, 1602-1605. https://doi.org/10.1109/IEMBS.2003.1279672

Hardwick, R. M., Rottschy, C., Miall, R. C., \& Eickhoff, S. B. (2013). A quantitative metaanalysis and review of motor learning in the human brain. Neuroimage, 67, 283297. https://doi.org/10.1016/j.neuroimage.2012.11.020

Hargrove, L., Englehart, K., \& Hudgins, B. (2008). A training strategy to reduce classification degradation due to electrode displacements in pattern recognition based myoelectric control. Biomed. Signal Process. Control, 3(2), 175-180. https://doi.org/10.1016/j.bspc.2007.11.005

Hart, S. G., \& Staveland, L. E. (1988). Development of NASA-TLX (Task Load Index): Results of empirical and theoretical research. Adv. Psychol., 52, 139-183. Retrieved from http://www.sciencedirect.com/science/article/pii/S0166411508623869

Henderson, L., Gustin, S., Macey, P., Wrigley, P., \& Siddall, P. (2011). Functional reorganization of the brain in humans following spinal cord injury: evidence for underlying changes in cortical anatomy. J. Neurosci., 31(7), 2630-2637. https://doi.org/10.1523/JNEUROSCI.2717-10.2011

Hoffman, L. R., \& Field-Fote, E. C. (2007). Cortical reorganization following bimanual training and somatosensory stimulation in cervical spinal cord injury: a case report. Phys. Ther., 87(2), 208-223. https://doi.org/10.2522/ptj.20050365

Hotz-Boendermaker, S., Funk, M., Summers, P., Brugger, P., Hepp-Reymond, M. C., Curt, A., \& Kollias, S. S. (2008). Preservation of motor programs in paraplegics as demonstrated by attempted and imagined foot movements. Neuroimage, 39(1), 383-394. https://doi.org/10.1016/j.neuroimage.2007.07.065

Howard, J. D., Hoit, J. D., Enoka, R. M., \& Hasan, Z. (1986). Relative activation of two human elbow flexors under isometric conditions: a cautionary note concerning flexor equivalence. Exp. Brain Res., 62(1), 199-202. https://doi.org/10.1007/BF00237416

Huang, D., Qian, K., Fei, D. Y., Jia, W., Chen, X., \& Bai, O. (2012). Electroencephalography (EEG)-based brain-computer interface (BCI): A 2-D virtual wheelchair control based on event-related desynchronization/ synchronization and state control. IEEE Trans. Neural Syst. Rehabil. Eng., 20(3), 379-388. https://doi.org/10.1109/TNSRE.2012.2190299

Hussaini, A., \& Kyberd, P. (2016). Refined clothespin relocation test and assessment of motion. Prosthet Orthot Int. https://doi.org/10.1177/0309364616660250

Iturrate, I., Antelis, J., \& Minguez, J. (2009). Synchronous EEG Brain-Actuated Wheelchair with Automated Navigation. Icra 2009 Ieee Int. Conf. Robot. Autom. Vols 1-7, 2530- 
2537.

Jiang, N., Dosen, S., Farina, D., Signal, I., Magazine, P., Jiang, N., ... Farina, D. (2012). Myoelectric Control of Artificial Limbs: is There the Need for a Change of Focus? IEEE Signal Process. Mag., 29(5), 147-150. https://doi.org/10.1109/Msp.2012.2203480

Jiang, N., Dosen, S., Muller, K. R., \& Farina, D. (2012). Myoelectric Control of Artificial Limbs-Is There a Need to Change Focus? IEEE Signal Process. Mag., 29(5), 147-150. https://doi.org/10.1109/Msp.2012.2203480

Jiang, N., Muceli, S., Graimann, B., \& Farina, D. (2013). Effect of arm position on the prediction of kinematics from EMG in amputees. Med. Biol. Eng. Comput., 51(1-2), 143-151. https://doi.org/10.1007/s11517-012-0979-4

Johansen, D., Sebelius, F., Jensen, S., Bentsen, B., Popovic, D. B., \& Andreasen Struijk, L. N. (2016). A comparative study of virtual hand prosthesis control using an inductive tongue control system. Assist Technol, 28(1), 22-29. https://doi.org/10.1080/10400435.2015.1070303

Jurkiewicz, M. T., Mikulis, D. J., McIlroy, W. E., Fehlings, M. G., \& Verrier, M. C. (2007). Sensorimotor Cortical Plasticity During Recovery Following Spinal Cord Injury: A Longitudinal fMRI Study. Neurorehabil. Neural Repair, 21(6), 527-538. https://doi.org/10.1177/1545968307301872

Karl, A., Birbaumer, N., Lutzenberger, W., Cohen, L. G., \& Flor, H. (2001). Reorganization of motor and somatosensory cortex in upper extremity amputees with phantom limb pain. J. Neurosci., 21(10), 3609-18. https://doi.org/21/10/3609 [pii]

Karni, A., Meyer, G., Jezzard, P., Adams, M. M., Turner, R., \& Ungerleider, L. G. (1995). Functional MRI evidence for adult motor cortex plasticity during motor skill learning. Nature, 377(6545), 155-8. https://doi.org/10.1038/377155a0

Karni, A., Meyer, G., Rey-Hipolito, C., Jezzard, P., Adams, M. M., Turner, R., \& Ungerleider, L. G. (1998). The acquisition of skilled motor performance: Fast and slow experience-driven changes in primary motor cortex. Proc. Natl. Acad. Sci., 95(3), 861-868. https://doi.org/10.1073/pnas.95.3.861

Kauhanen, L., Nykopp, T., Lehtonen, J., Jylanki, P., Heikkonen, J., Rantanen, P., ... Sams, M. (2006). EEG and MEG Brain Computer Interface for Tetraplegic Patients. IEEE Trans. Neural Syst. Rehabil. Eng., 14(2), 190-193.

Kiang, N.-S., Crist, A. H., French, M. A., \& Edwards, A. G. (1963). Postauricular electric response to acoustic stimuli in humans. Q. Prog. Report, Massachusetts Inst. Technol., 218- 225.

Kim, J., Park, H., Bruce, J., Sutton, E., Rowles, D., Pucci, D., ... Ghovanloo, M. (2013). The tongue enables computer and wheelchair control for people with spinal cord injury. Sci. Transl. Med., 5(213), 166-213. https://doi.org/10.1126/scitranslmed.3006296

Kirshblum, S. C., Burns, S. P., Biering-Sorensen, F., Donovan, W., Graves, D. E., Jha, A., ... Waring, W. (2011). International standards for neurological classification of spinal cord injury (revised 2011). J Spinal Cord Med, 34(6), 535-546. https://doi.org/10.1179/204577211X13207446293695

Kokotilo, K. J., Eng, J. J., \& Curt, A. (2009). Reorganization and Preservation of Motor Control of the Brain in Spinal Cord Injury: A Systematic Review. J Neurotrauma, 
26(11), 2113-2126. https://doi.org/10.1089/neu.2008.0688

Larson, E., Terry, H. P., \& Stepp, C. E. (2012). Audio-visual feedback for electromyographic control of vowel synthesis. In Proceedings of the Annual International Conference of the IEEE Engineering in Medicine and Biology Society, EMBS (pp. 3600-3603). https://doi.org/10.1109/EMBC.2012.6346745

Lebedev, M. A., \& Nicolelis, M. A. L. (2006). Brain-machine interfaces: past, present and future. Trends Neurosci., 29(9), 536-546. https://doi.org/10.1016/j.tins.2006.07.004

Levy, W. J., Amassian, V. E., Traad, M., \& Cadwell, J. (1990). Focal magnetic coil stimulation reveals motor cortical system reorganized in humans after traumatic quadriplegia. Brain Res., 510(1), 130-134. https://doi.org/10.1016/00068993(90)90738-W

Lim, P., \& Tow, A. (2007). Recovery and Regeneration after Spinal Cord Injury: A Review and Summary of Recent Literature. Ann. Acad. Med. Singapore, 36(1), 49-57.

Lohse, K. R., Wadden, K., Boyd, L. A., \& Hodges, N. J. (2014). Motor skill acquisition across short and long time scales: A meta-analysis of neuroimaging data. Neuropsychologia, 59(1), 130-141. https://doi.org/10.1016/j.neuropsychologia.2014.05.001

Lontis, E. R., Lund, M. E., Christensen, H. V, Bentsen, B., Gaihede, M., Caltenco, H. A., \& Andreasen Struijk, L. N. (2010). Clinical evaluation of wireless inductive tongue computer interface for control of computers and assistive devices. Conf Proc IEEE Eng Med Biol Soc, 2010, 3365-3368. https://doi.org/10.1109/IEMBS.2010.5627924

Losier, Y., Englehart, K., \& Hudgins, B. (2011). Evaluation of shoulder complex motionbased input strategies for endpoint prosthetic-limb control using dual-task paradigm. J. Rehabil. Res. Dev., 48(6), 669-678. https://doi.org/10.1682/Jrrd.2010.08.0165

Lotze, M., Braun, C., Birbaumer, N., Anders, S., \& Cohen, L. G. (2003). Motor learning elicited by voluntary drive, Brain, 126(4), 866-872. https://doi.org/10.1093/brain/awg079

Lotze, M., Laubis-Herrmann, U., \& Topka, H. (2006). Combination of TMS and fMRI reveals a specific pattern of reorganization in M1 in patients after complete spinal cord injury. Restor. Neurol. Neurosci., 24(2), 97-107.

Lotze, M., Laubis-Herrmann, U., Topka, H., Erb, M., \& Grodd, W. (1999). Reorganization in the primary motor cortex after spinal cord injury - A functional Magnetic Resonance (fMRI) study. Restor. Neurol. Neurosci., 14(2-3), 183-187. Retrieved from http://www.scopus.com/inward/record.url?eid=2-s2.00032935973\&partnerID=40\&md5=520515c87b3a7d7994dc6d666be5d52c

Lundell, H., Barthelemy, D., Skimminge, A., Dyrby, T. B., Biering-Sørensen, F., \& Nielsen, J. B. (2011). Independent spinal cord atrophy measures correlate to motor and sensory deficits in individuals with spinal cord injury. Spinal Cord, 49(1), 70-75. https://doi.org/10.1038/sc.2010.87

Lundell, H., Christensen, M. S., Barthélemy, D., Willerslev-Olsen, M., Biering-Sørensen, F., \& Nielsen, J. B. (2011). Cerebral activation is correlated to regional atrophy of the spinal cord and functional motor disability in spinal cord injured individuals. 
Neuroimage,

$54(2)$,

1254-1261.

https://doi.org/10.1016/j.neuroimage.2010.09.009

Mainardi, E., \& Davalli, A. (2007). Controlling a prosthetic arm with a throat microphone. Conf Proc IEEE Eng Med Biol Soc, 2007, 3035-3039. https://doi.org/10.1109/IEMBS.2007.4352968

Mathiowetz, V., Volland, G., Kashman, N., \& Weber, K. (1985). Adult Norms for the Box and Block Test of Manual Dexterity. Am. J. Occup. Ther., 39(6), 386-391. Retrieved from http://ajot.aota.org/article.aspx?articleid=1884839

Mattia, D., Cincotti, F., Mattiocco, M., Scivoletto, G., Marciani, M. G., \& Babiloni, F. (2006). Motor-related cortical dynamics to intact movements in tetraplegics as revealed by high-resolution EEG. Hum. Brain Mapp., 27(6), 510-519. https://doi.org/10.1002/hbm.20195

Meier, R. H. (2004). History of Arm Amputation, Prosthetic Restoration, and Arm Amputation Rehabilitation. Funct. Restor. Adults Child. with Up. Extrem. Amputation, $1-7$.

Meincke, J., Hewitt, M., Reischl, M., Rupp, R., Schmidt-Samoa, C., \& Liebetanz, D. (n.d.). Cortical Representation of Auricular Muscles in Humans: A Robot-Controlled TMS Mapping and fMRI Study. In Preparation.

Middleton, J., Tran, Y., \& Craig, A. (2007). Relationship between quality of life and selfefficacy in persons with spinal cord injuries. Arch. Phys. Med. Rehabil., 88(12), 16431648. https://doi.org/10.1016/j.apmr.2007.09.001

Mikulis, D. J., Jurkiewicz, M. T., McIlroy, W. E., Staines, W. R., Rickards, L., Kalsi-Ryan, S., ... Verrier, M. C. (2002). Adaptation in the motor cortex following cervical spinal cord injury. Neurology, 58(5), 794-801. https://doi.org/10.1212/WNL.58.5.794

Millán, J. D. R., Galan, F., Vanhooydonck, D., Lew, E., Philips, J., Nuttin, M., ... Nuttin, M. (2009). Asynchronous non-invasive brain-actuated control of an intelligent wheelchair. Proc. Annu. Int. Conf. IEEE Eng. Med. Biol. Soc. EMBS, 3361-3364. https://doi.org/10.1109/IEMBS.2009.5332828

Millán, J. D., Rupp, R., Müller-Putz, G. R., Murray-Smith, R., Giugliemma, C., Tangermann, M., ... Mattia, D. (2010). Combining Brain-Computer Interfaces and Assistive Technologies: State-of-the-Art and Challenges. Front Neurosci, 4, 1-16. https://doi.org/10.3389/fnins.2010.00161

Milton, J., Solodkin, A., Hluštík, P., \& Small, S. L. (2007). The mind of expert motor performance is cool and focused. Neuroimage, 35(2), 804-813. https://doi.org/10.1016/j.neuroimage.2007.01.003

Moxon, K. A., Oliviero, A., Aguilar, J., \& Foffani, G. (2014). Cortical reorganization after spinal cord injury: Always for good? Neuroscience, 283, 78-94. https://doi.org/10.1016/j.neuroscience.2014.06.056

Muzumdar, A. (2004). Powered upper limb prostheses: control, implementation and clinical application. Springer.

Nardone, R., Höller, Y., Brigo, F., Orioli, A., Tezzon, F., Schwenker, K., ... Trinka, E. (2015). Descending motor pathways and cortical physiology after spinal cord injury assessed by transcranial magnetic stimulation: a systematic review. Brain Res., 1619, 139-154. https://doi.org/10.1016/j.brainres.2014.09.036 
Nardone, R., Höller, Y., Brigo, F., Seidl, M., Christova, M., Bergmann, J., ... Trinka, E. (2013). Functional brain reorganization after spinal cord injury: Systematic review of animal and human studies. Brain Res., 1504, 58-73. https://doi.org/10.1016/j.brainres.2012.12.034

National SCI Statistical Center. (2015). Spinal cord injury facts and figures at a glance. J. Spinal Cord Med., 33(August), 439-440.

Niemi, P., \& Naatanen, R. (1981). Foreperiod and Simple Reaction-Time. Psychol. Bull., 89(1), 133-162. https://doi.org/Doi 10.1037//0033-2909.89.1.133

Oldfield, R. C. (1971). The assessment and analysis of handedness: the Edinburgh inventory. Neuropsychologia, 9(1), 97-113. Retrieved from http://www.ncbi.nlm.nih.gov/pubmed/5146491

Ortiz-Rosario, A., \& Adeli, H. (2013). Brain-computer interface technologies: From signal to action. Rev. Neurosci., 24(5), 537-552. https://doi.org/10.1515/revneuro-20130032

Perez-Maldonado, C., Wexler, A. S., \& Joshi, S. S. (2010). Two-Dimensional Cursor-toTarget Control From Single Muscle Site sEMG Signals. IEEE Trans. Neural Syst. Rehabil. Eng., 18(2), 203-209. https://doi.org/10.1109/TNSRE.2009.2039394

Perez, M. A., Lungholt, B. K. S., Nyborg, K., \& Nielsen, J. B. (2004). Motor skill training induces changes in the excitability of the leg cortical area in healthy humans. Exp. Brain Res., 159(2), 197-205. https://doi.org/10.1007/s00221-004-1947-5

Pfurtscheller, G., Müller, G. R., Pfurtscheller, J., Gerner, H. J., \& Rupp, R. (2003). "Thought" - Control of functional electrical stimulation to restore hand grasp in a patient with tetraplegia. Neurosci. Lett., 351(1), 33-36. https://doi.org/10.1016/S03043940(03)00947-9

Pistohl, T., Cipriani, C., Jackson, A., \& Nazarpour, K. (2013). Abstract and proportional myoelectric control for multi-fingered hand prostheses. Ann Biomed Eng, 41(12), 2687-2698. https://doi.org/10.1007/s10439-013-0876-5

Plotkin, A., Sela, L., Weissbrod, A., Kahana, R., Haviv, L., Yeshurun, Y., ... Sobel, N. (2010). Sniffing enables communication and environmental control for the severely disabled. Proc Natl Acad Sci $U$ S A, 107(32), 14413-14418. https://doi.org/10.1073/pnas.1006746107

Poldrack, R. A. (2000). Imaging Brain Plasticity: Conceptual and Methodological IssuesA Theoretical Review. Neuroimage, 12(1), 1-13. https://doi.org/10.1006/nimg.2000.0596

Porro, C. A., Francescato, M. P., Cettolo, V., Diamond, M. E., Baraldi, P., Zuiani, C., ... di Prampero, P. E. (1996). Primary motor and sensory cortex activation during motor performance and motor imagery: a functional magnetic resonance imaging study. J. Neurosci., 16(23), 7688-7698. https://doi.org/8922425

Post, M. W., van Asbeck, F. W., van Dijk, a J., \& Schrijvers, a J. (1997). Services for spinal cord injured: availability and satisfaction. Spinal Cord Off. J. Int. Med. Soc. Paraplegia, 35(2), 109-115. https://doi.org/10.1038/sj.sc.3100362

Powell, M. A., \& Thakor, N. V. (2013). A Training Strategy for Learning Pattern Recognition Control for Myoelectric Prostheses. J. Prosthet. Orthot., 25(1), 30-41. https://doi.org/10.1097/JP0.0b013e31827af7c1 
Power, J. D., Mitra, A., Laumann, T. O., Snyder, A. Z., Schlaggar, B. L., \& Petersen, S. E. (2014). Methods to detect, characterize, and remove motion artifact in resting state

Neuroimage,

84

320-341. https://doi.org/10.1016/j.neuroimage.2013.08.048

Raineteau, 0., \& Schwab, M. E. (2001). Plasticity of motor systems after incomplete spinal cord injury. Nat. Rev. Neurosci., 2(4), 263-273. https://doi.org/10.1038/35067570

Reilly, K. T., \& Sirigu, A. (2008). The motor cortex and its role in phantom limb phenomena. Neuroscientist, 14(2), 195-202. https://doi.org/10.1177/1073858407309466

Rouse, E. J., Nahlik, D. C., Peshkin, M. A., \& Kuiken, T. A. (2011). Development of a Model Osseo-Magnetic Link for Intuitive Rotational Control of Upper-Limb Prostheses. Ieee Trans. Neural Syst. Rehabil. Eng., 19(2), 213-220. https://doi.org/10.1109/Tnsre.2010.2102365

Roy, F. D., Zewdie, E. T., \& Gorassini, M. A. (2011). Short-interval intracortical inhibition with incomplete spinal cord injury. Clin. Neurophysiol., 122(7), 1387-1395. https://doi.org/10.1016/j.clinph.2010.11.020

Rubio, S., Diaz, E., Martin, J., \& Puente, J. M. (2004). Evaluation of subjective mental workload: A comparison of SWAT, NASA-TLX, and workload profile methods. Appl. Psychol. Int. Rev. Appl. Int., 53(1), 61-86. https://doi.org/Doi 10.1111/J.14640597.2004.00161.X

Ryu, S. I., \& Shenoy, K. V. (2009). Human cortical prostheses: lost in translation? Neurosurg Focus, 27(1), E5. https://doi.org/10.3171/2009.4.FOCUS0987

Sabbah, P., Schonen, S. De, Leveque, C., Gay, S., Pfefer, F., Nioche, C., ... Cordoliani, Y. (2002). Sensorimotor Cortical Activity in Patients with Complete Spinal Cord Injury : A Functional Magnetic Resonance Imaging Study. J. Neurotrauma, 19(1).

Sabre, L., Tomberg, T., Kõrv, J., Kepler, J., Kepler, K., Linnamägi, Ü., \& Asser, T. (2013). Brain activation in the acute phase of traumatic spinal cord injury. Spinal Cord, 51(8), 623-629. https://doi.org/10.1038/sc.2013.41

Scheme, E., \& Englehart, K. (2011). Electromyogram pattern recognition for control of powered upper-limb prostheses: state of the art and challenges for clinical use. J. Rehabil. Res. Dev., 48(6), 643-659.

Scherer, M. J., \& Cushman, L. A. (2001). Measuring subjective quality of life following spinal cord injury: A validation study of the assistive technology device predisposition assessment. Disabil. Rehabil., 23(9), 387-393. https://doi.org/10.1080/09638280010006665

Schmalfuss, L., Hahne, J., Farina, D., Hewitt, M., Kogut, A., Doneit, W., ... Liebetanz, D. (n.d.). A hybrid auricular control system: direct, simultaneous, and proportional myoelectric control of two degrees of freedom in prosthetic hands.

Schmalfuss, L., Rupp, R., Tuga, M. R., Kogut, A., Hewitt, M., Meincke, J., ... Liebetanz, D. (2016). Steer by ear: Myoelectric auricular control of powered wheelchairs for individuals with spinal cord injury. Restor. Neurol. Neurosci., 34(1), 79-95. https://doi.org/10.3233/RNN-150579

Schmalfuß, L., Rupp, R., Tuga, M. R., Kogut, A., Hewitt, M., Meincke, J., ... Liebetanz, D. 
(2015). Steer by ear: Myoelectric auricular control of powered wheelchairs for individuals with spinal cord injury. Restor. Neurol. Neurosci., 34(1), 79-95. https://doi.org/10.3233/RNN-150579

Serra, G., Tugnoli, V., Cristofori, M. C., Eleopra, R., \& De Grandis, D. (1986). The electromyographic examination of the posterior auricular muscle. Electromyogr Clin Neurophysiol, 26(8), 661-665. Retrieved from http://www.ncbi.nlm.nih.gov/pubmed/3830046

Shoham, S., Halgren, E., Maynard, E. M., \& Normann, R. a. (2001). Motor-cortical activity in tetraplegics. Nature, 413(6858), 793. https://doi.org/10.1038/35101651

Silva, N. A., Sousa, N., Reis, R. L., \& Salgado, A. J. (2014). From basics to clinical: A comprehensive review on spinal cord injury. Prog. Neurobiol., 114, 25-57. https://doi.org/10.1016/j.pneurobio.2013.11.002

Simpson, R. C., \& Levine, S. P. (2002). Voice control of a powered wheelchair. IEEE Trans Neural Syst Rehabil Eng, 10(2), 122-125. https://doi.org/10.1109/TNSRE.2002.1031981

Struijk, L. N. (2006). An inductive tongue computer interface for control of computers and assistive devices. IEEE Trans Biomed Eng, 53(12), 2594-2597. https://doi.org/10.1109/TBME.2006.880871

Struijk, L. N., Lontis, E. R., Bentsen, B., Christensen, H. V, Caltenco, H. A., \& Lund, M. E. (2009). Fully integrated wireless inductive tongue computer interface for disabled people. Conf Proc IEEE Eng Med Biol Soc, 2009, 547-550. https://doi.org/10.1109/IEMBS.2009.5333192

Tanaka, K., Matsunaga, K., \& Wang, H. O. (2005). Electroencephalogram-based control of an electric wheelchair. Ieee Trans. Robot., 21(4), 762-766. https://doi.org/Doi 10.1109/Tro.2004.842350

Theofrastous, J. P., Wyman, J. F., Bump, R. C., McClish, D. K., Elser, D. M., Bland, D. R., \& Fantl, J. A. (2002). Effects of pelvic floor muscle training on strength and predictors of response in the treatment of urinary incontinence, 21(5), 486-490. https://doi.org/10.1002/nau.10021

Torkia, C., Reid, D., Korner-Bitensky, N., Kairy, D., Rushton, P. W., Demers, L., \& Archambault, P. S. (2015). Power wheelchair driving challenges in the community: a users' perspective. Disabil. Rehabil. Assist. Technol., 10(3), 211-215. https://doi.org/10.3109/17483107.2014.898159

Turner, J. a, Lee, J. S., Schandler, S. L., \& Cohen, M. J. (2003). An fMRI investigation of hand representation in paraplegic humans. Neurorehabil. Neural Repair, 17, 37-47. https://doi.org/10.1177/0888439002250443

Ungerleider, L. (2002). Imaging Brain Plasticity during Motor Skill Learning. Neurobiol. Learn. Mem., 78(3), 553-564. https://doi.org/10.1006/nlme.2002.4091

Velliste, M., Perel, S., Spalding, C., Whitford, A., Schwartz, A., \& Mckhann, G. M. (2008). Cortical Control of a Prosthetic Arm for Self-feeding. Nature, 453(2), 1098-1101. https://doi.org/10.1227/01.NEU.0000335793.88007.CE

Vidovic, M. M.-C., Hwang, H.-J., Amsuss, S., Hahne, J. M., Farina, D., \& Muller, K.-R. (2016). Improving the Robustness of Myoelectric Pattern Recognition for Upper Limb Prostheses by Covariate Shift Adaptation. IEEE Trans. Neural Syst. Rehabil. Eng., 
24(9), 961-970. https://doi.org/10.1109/TNSRE.2015.2492619

Weir, R. F., Troyk, P. R., DeMichele, G., \& Kerns, D. (2005). Technical Details of the Implantable Myoelectric Sensor (IMES) System for Multifunction Prosthesis Control. Conf Proc IEEE Eng Med Biol Soc., 7337-7340. https://doi.org/10.1109/IEMBS.2005.1616206

Wilson, S. A. K. (1908). A note on an associated movement of the eyes and ears in man. Rev Neurol Psychiatry, 6, 331-336.

Winchester, P., McColl, R., Querry, R., Foreman, N., Mosby, J., Tansey, K., \& Williamson, J. (2005). Changes in supraspinal activation patterns following robotic locomotor therapy in motor-incomplete spinal cord injury. Neurorehabil. Neural Repair, 19(4), 313-324. https://doi.org/10.1177/1545968305281515

Wolpaw, J. R. R., Birbaumer, N., McFarland, D. J. J., Pfurtscheller, G., \& Vaughan, T. M. M. (2002). Brain-computer interfaces for communication and control. Clin Neurophysiol, 113(6), 767-791. https://doi.org/S1388245702000573 [pii]

Wright, T. W., Hagen, A. D., \& Wood, M. B. (1995). Prosthetic Usage in Major Upper Extremity Amputations. J. Hand Surgery-American Vol., 20a(4), 619-622. https://doi.org/Doi 10.1016/S0363-5023(05)80278-3

Wrigley, P. J., Gustin, S. M., Macey, P. M., Nash, P. G., Gandevia, S. C., Macefield, V. G., ... Henderson, L. A. (2009). Anatomical changes in human motor cortex and motor pathways following complete thoracic spinal cord injury. Cereb. Cortex, 19(1), 224232. https://doi.org/10.1093/cercor/bhn072

Wyndaele, M., \& Wyndaele, J.-J. (2006). Incidence, prevalence and epidemiology of spinal cord injury: what learns a worldwide literature survey? Spinal Cord, 44(9), 523529. https://doi.org/10.1038/sj.sc.3101893

Youdin, M., Sell, G. H., Reich, T., Clagnaz, M., Louie, H., \& Kolwicz, R. (1980). A voice controlled powered wheelchair and environmental control system for the severely disabled. Med Prog Technol, 7(2-3), 139-143. Retrieved from http://www.ncbi.nlm.nih.gov/pubmed/7393174

Young, A. J., Hargrove, L. J., Kuiken, T., \& others. (2011). The effects of electrode size and orientation on the sensitivity of myoelectric pattern recognition systems to electrode shift. IEEE Trans Biomed Eng, 58(9), 2537-2544.

Yousefi, B., Huo, X., \& Ghovanloo, M. (2011). Preliminary assessment of Tongue Drive System in medium term usage for computer access and wheelchair control. Conf Proc IEEE Eng Med Biol Soc, 2011, 5766-5769. https://doi.org/10.1109/IEMBS.2011.6091427

Yousefi, B., Huo, X., Kim, J., Veledar, E., \& Ghovanloo, M. (2012). Quantitative and comparative assessment of learning in a tongue-operated computer input device-part II: navigation tasks. IEEE Trans Inf Technol Biomed, 16(4), 633-643. https://doi.org/10.1109/TITB.2012.2191793

Zecca, M., Micera, S., Carrozza, M. C., \& Dario, P. (2002). Control of multifunctional prosthetic hands by processing the electromyographic signal. Crit Rev Biomed Eng, 30(4-6), 459-485.

Ziegler-Graham, K., MacKenzie, E. J., Ephraim, P. L., Travison, T. G., \& Brookmeyer, R. (2008). Estimating the prevalence of limb loss in the United States: 2005 to 2050. 
$\begin{array}{llll}\text { Arch Phys } \quad \text { Med } & \text { Rehabil, } & \text { 422-429. }\end{array}$ https://doi.org/10.1016/j.apmr.2007.11.005

Ziemann, U., Corwell, B., \& Cohen, L. G. (1998). Modulation of plasticity in human motor cortex after forearm ischemic nerve block. J. Neurosci., 18(3), 1115-1123. https://doi.org/10.1155/2012/913472 


\section{List of abbreviations}

ACS

auricular control system

ASIA

american Spinal Injury Association

ANOVA

analysis of Variance

ATD-PA

assistive Technology Device Predisposition Assessment

BCI

brain computer interface

BDNF

brain-derived neurotrophic factor

BOLD

blood-oxygen-level-dependent

CC

co-contraction control

CNS

central nerve system

COG

centers of gravity

DOF

degree of freedom

EEG

electroencephalography

EMG

electromyography

EPI

echo-planar imaging

EW

ear-wiggler

e.g.

example given

FD

framewise displacement

Fig.

Figure

FT

finger tapping

FLIRT

FMRIB's linear image registration tool

fMRI

functional magnetic resonance imaging

FNIRT

FMRIB's non-linear image registration tool

FSL

FMRIB's software library

FWHM

full-width half-maximum

GLM

general linear model

$\mathrm{h}$

hour

hACS

hybrid auricular control system

$\mathrm{Hz}$

Hertz

i.e.

id est

$\mathrm{kHz}$

kilohertz 


\begin{tabular}{|c|c|}
\hline $\mathrm{LH}$ & left brain hemisphere \\
\hline M & mean \\
\hline M1 & primary motor cortex \\
\hline MEP & motor evoked potentials \\
\hline MNI & Montreal Neurological Insitute and Hospital \\
\hline ms & milliseconds \\
\hline $\mathrm{N}$ & newton \\
\hline NASA TLX & national Aeronautics and Space Administration Task Load Index \\
\hline NW & non-ear-wiggler \\
\hline PAM & posterior auricular muscle \\
\hline RH & right brain hemisphere \\
\hline ROI & region of interest \\
\hline s & seconds \\
\hline SEM & standard error of mean \\
\hline S1 & primary sensory cortex \\
\hline SCI & spinal cord injury \\
\hline SD & standard deviation \\
\hline sEMG & surface elektromyography \\
\hline SL & slope control \\
\hline SMA & supplementary motor area \\
\hline SPSS & Statistical package for the social sciences \\
\hline TMS & transcranial magnetic stimulation \\
\hline $\mathrm{TE}$ & echo time \\
\hline TR & repetition time \\
\hline$\mu \mathrm{m}$ & micrometer \\
\hline
\end{tabular}




\section{List of tables}

Table 1) Clinical information on the ten study participants.

Table 2) Talairach coordinates of centers of gravity (COGs) and activation cluster sizes of task-related activations in fMRI (in $\mathrm{mm} 3$ with standard deviations) in the primary motor cortex.

Supp. Table 1) Summary of results and statistics of the PAM performance criteria in the first study.

\section{List of figures}

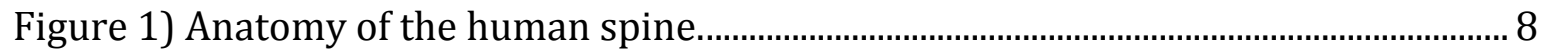

Figure 2) Individual with tetraplegia using a chin control...................................................10

Figure 3) The motor cortex (green) on a human brain with areas typically deefferented in individuals with tetraplegia (red). ................................................................13

Figure 4) Arm amputation levels (A) and a common prosthetic hand (B)......................19

Figure 5) Anatomy of the human auricular muscles. .........................................................24

Figure 6) Setup of the auricular control system (ACS).........................................................30

Figure 7) Diagram of signal processing in the ACS. ...............................................................

Figure 8) Signal processing in the user interface. ...................................................................36

Figure 9) The real obstacle course for testing the performance of the ACS in

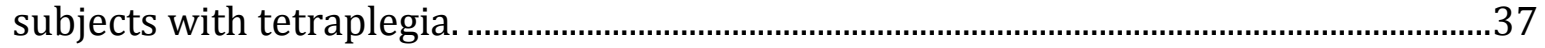

Figure 10) Positive training effects on PAM activation.........................................................41

Figure 11) Improved performance using the ACS in a virtual obstacle course...............42

Figure 12) Training effects of subjects with tetraplegia........................................................44

Figure 13) Real and virtual wheelchair driving using the ACS...........................................45

Figure 14) Set-up of the auricular control system (A) and signal generation for

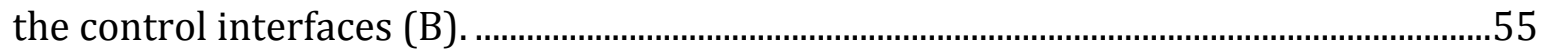

Figure 15) Speed and errors in ten able-bodied subjects in two-DOF clothespin

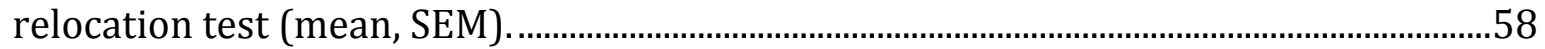

Figure 16) Results in one-DOF box and blocks test (mean, SEM) .....................................58

Figure 17) Influence of arm position on performance in the clothespin test (mean,

SEM)

Figure 18) Training effect in the clothespin relocation test (mean, SEM).......................60

Figure 19) Results of the transradial amputee in the clothespin relocation test

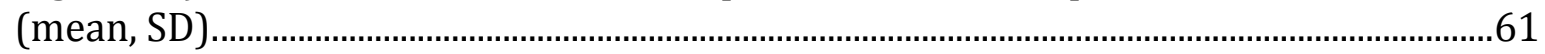

Figure 20) Wheelchair set-up with the auricular control system (ACS) ..........................68

Figure 21) Effects of training on the control capacity of the PAM. .....................................74

Figure 22) Centers of gravity (COG) of PAM and FT fMRI representations of SCI

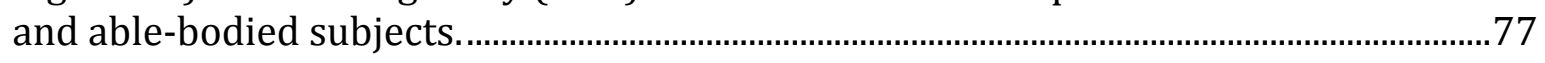

Figure 23) Reduction of motor-related activation cluster size in fMRI through

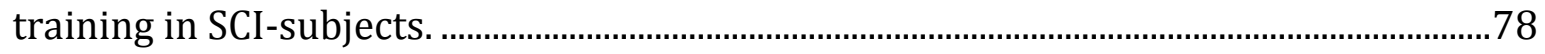

Figure 24) Whole brain group analysis of during PAM activation pre-training

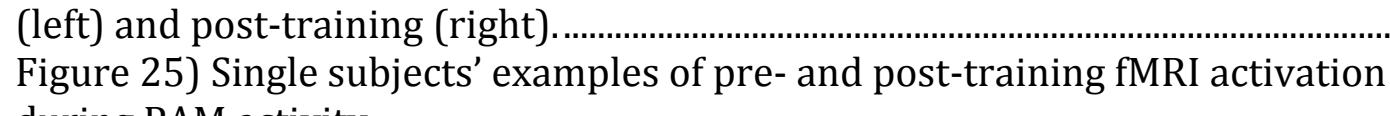
during PAM activity. .80 
Figure 26) Spearman correlations of fMRI activation cluster sizes during PAM activity pre- and post-training.

Figure 27) Centers of Gravity (COGs) of TMS motor evoked potentials of the right

PAM.

Supp. Fig. 1) Training PAM activation - car race.

Supp. Fig. 2) Training PAM activation - collecting coins..

Supp. Fig. 3) Test for reaction times (RT) of contraction and relaxation.

105

Supp. Fig. 4) Test for maximum contraction duration.

106

Supp. Fig. 5) Test for graded PAM activity.

106

Supp. Fig. 6) Test for lateralized activation.

Supp. Fig. 7) Training effects for subgroups of ear-wigglers and non ear-wigglers...108 


\section{Contributions}

In the first study, the study design, able-bodied subjects recruitment, training and testing of the subjects as well as data analysis was done by myself. I also wrote the manuscript, which was published in Restorative Neuroscience and Neurology (Schmalfuß et al., 2015).

All co-authors read the final draft and made suggestions for corrections. Chapter 2.1.1 was edited by Rüdiger Rupp as well as Figure 8; chapter 2.1.2 was edited by Markus Reischl. Rüdiger Rupp, Andreas Kogut and Ute Eck developed the hardware of the ACS. Markus Reischl, Michele Tuga and Ralf Mikut developed the software. Manuel Hewitt, Jonna Meincke, Willi Duttenhoefer and Florian Klinker were involved in earlier developmental steps of the ACS.

In the second study, the study design, able-bodied subjects recruitment, training and testing of the subjects as well as data analysis was done by myself. I also wrote the manuscript, which is prepared for publication (Schmalfuss et al., n.d.).

All co-authors read the final draft and made suggestions for corrections. Figure 1a was created with contribution of Manuel Hewitt, Figure $1 \mathrm{~b}$ was created with contribution of Janne Hahne. Again Rüdiger Rupp and Andreas Kogut were responsible for the hardware and Markus Reischl and Wolfgang Doneit for the software. Dario Farina and Janne Hahne were involved in the study design. Janne Hahne was responsible for the development of the hybrid auricular control system and the technical assistance during data acquisition.

In the third study, I designed the study, recruited the subjects, organized the logistics of the study (e.g. hospitalization, transport) and trained all subjects. I collected and analyzed the data, created all figures and wrote the manuscript.

Again Rüdiger Rupp and Andreas Kogut were responsible for the hardware and Markus Reischl and Wolfgang Doneit for the software. Carsten Schmidt-Samoa contributed to fMRI data acquisition and analysis and edited chapter 4.1.5. Manuel Hewitt contributed to TMS data acquisition and analysis.

All studies were supervised by David Liebetanz. 


\section{Acknowledgments}

This thesis was funded by the German Federal Ministry of Education and Research (BMBF), grant numbers 01EZ1122A, 01EZ1122B and 01EZ1123C and by the Göttingen Graduate School for Neuorsciences and Biology (GGNB) with a three month bridging fund.

First of all, I would like to thank my supervisor David Liebetanz for being there every step of the way. This work is based on his ideas and would not have been possible without his exceptional support.

To all our subjects who dedicated their time and enthusiasm to this project: thanks for your cooperation despite all the handicaps, your effort made this thesis possible.

Thanks to the teams from Karlsruhe and Heidelberg for the great working atmosphere, the support and for making work meetings a thing to look forward to. It was a pleasure working with you.

I thank the teams at the Carolinum, Bad Karlshafen and the Orthopädische Klinik Hessisch Lichtenau, especially Prof. Saur, Jürgen Vollgraff and Diana Ehmer, for the support of our idea an the extensive logistic support of our study.

I am very grateful to Carsten Schmidt-Samoa for his tireless advices on all aspects of MRI, and for Ilona Pfahlert and Britta Perl for the assistance and welcoming environment.

I would like to thank my colleagues at the department for the support and the fun and loyal atmosphere, that made it easy coming back when I had been away: Zsolt Turi, Ivan Alekseichuk, Annika Primaßin, Gabriel Amador. Thanks to Giorgi Badzikadzi, who proved to be an ear-wiggling-pro and patiently borrowed us his talent for testing the ACS. A special thanks goes to Manuel Hewitt for always being able to help with any kind of questions. Thanks to Marion Kurze for all the help with bureaucracy and the support. I am grateful to Prof. Paulus for supporting me when funding ran out.

My deepest gratitude goes to all the persons in my life that have made the last years worth it: my mother and father for the encouraging support, emotional and logistical, throughout the years. Moritz, Kerstin and Greta for bringing me back to the ground. Leena and Nada for unconditionally going through all the ups and downs with me. To all 
my friends, near and far, and to my Bib-Crew for making writing this thesis fun. To Emiliano, for fighting your impatience, for sacrificing what was dearest to you, and for showing me that beauty lies behind the rocks on the way. And finally Mino, who is the center of everything. I could not have made it without you all. 


\section{Statement of Originality}

I hereby declare that this thesis is my own work and has been written independently with no other sources and aids than quoted in the text, contributions, references and acknowledgements.

Göttingen, December 2017

Leonie Schmalfuß 


\section{Curriculum Vitae}

Leonie Schmalfuß

* 15.02.1985

Lauchstädter Straße 21

04229 Leipzig

\section{EDUCATION}

Since $04 / 2013$

$10 / 2006-10 / 2012$

$10 / 2005-11 / 2011$

$05 / 2004$

\section{INTERNSHIPS}

$07 / 2009-10 / 2009$

$07 / 2010-09 / 2010$

$02 / 2010-04 / 2010$

WORK EXPERIENCE

$10 / 2008-10 / 2010$

RESEARCH ASSISTANT, University Mainz, Department of

PhD student, Clinic for Clinical Neurophysiology, University Medical Center, Göttingen

Göttingen Graduate School for Neurosciences, Biophysics, and Molecular Biosciences (GGNB)

Effects of training on the control capacity and the cortical representation of the human auricular muscle.

DIPLOMA PSYCHOLOGY, University Mainz

Diploma thesis at the Max-Planck-Institute for Cognitive and Brain Sciences, Leipzig:

TDCS-effects on executive functions: evidence from healthy subjects and TBI patients.

MAGISTER ARTIUM Hispanistik/ Philosophy/ Psychology, University Mainz

Thesis: Mexikanische Jugendsprache am Beispiel von Qualitätsausdrücken

ABITUR (Note 1,4), Georg-Christoph-Lichtenberg Gesamtschule Göttingen

\section{UNIVERSITY MEDICAL CENTER, GÖTTINGEN}

Clinic for Clinical Neurophysiology

HOSPITAL CIVIL GUADALAJARA, MEXIKO

Psychiatric department

MAX-PLANCK-INSTITUT FOR COGNITIVE AND BRAIN SCIENCES, LEIPZIG, Department of Neuropsychology 
Experimental Psychology

\section{PUBLISHED ARTICLES}

Nitsche, M. A., Jakoubkova, M., Thirugnanasambandam, N., Schmalfuss, L., Hullemann, S., Sonka, K., ... Happe, S. (2010). Contribution of the premotor cortex to consolidation of motor sequence learning in humans during sleep. J. Neurophysiol., 104(5). https://doi.org/10.1152/jn.00611.2010

Schmalfuß, L., Rupp, R., Tuga, M. R., Kogut, A., Hewitt, M., Meincke, J., ... Liebetanz, D. (2015). Steer by ear: Myoelectric auricular control of powered wheelchairs for individuals with spinal cord injury. Restor. Neurol. Neurosci., 34(1), 79-95. https://doi.org/10.3233/RNN-150579

Tuga, M. R., Rupp, R., Kogut, A., Liebetanz, D., Schmalfuss, L., Mikut, R., \& Reischl, M. (2014). Incremental parameter adaptation scheme for myoelectric-controlled human-machine interfaces. Biomed. Tech., 59. https://doi.org/10.1515/bmt-20145002

\section{CONFERENCE PROCEEDINGS}

Eck, U., Kogut, A., Tuga, M.R., Doneit, W., Schmalfuß, L., Liebetanz, D., Reischl, M., Rupp, R.: Steer by ear - Vergleichsstudie zur Steuerung eines Elektrorollstuhls mittels zweier oberflächlich gemessener Aktivierungsmuster der Ohrmuskulatur, Abstraktband der 29. Jahrestagung der Deutschsprachigen Gesellschaft für Paraplegie, 21, 2016

Rupp, R., Schmalfuß, L., Tuga, M., Kogut, A., Hewitt, M., Meincke, J., Duttenhöfer, W., Eck, U., Mikut, R., Reischl, M., Liebetanz, D.: TELMYOS - a telemetric wheelchair control interface based on the bilateral recording of myoelectric signals from ear muscles, Proceedings of the 5th Conference on Technically Assisted Rehabilitation (TAR), Berlin, 12.03.-13.03.2015

Rupp R., Schmalfuß L., Tuga M., Kogut A., Hewitt M., Eck U., Mikut R., Reischl M., Liebetanz D.: TELMYOS - a feasibility study of a telemetric wheelchair control interface based on the bilateral recording of myoelectric signals from ear muscles, ASIA 2016 Annual Scientific Meeting, Philadelphia, PA, April 13-16, 2016

Schmalfuß, L., Duttenhoefer, W., Meincke, J., Klinker, F., Hewitt, M., Tuga, M., Kogut, A., Reischl, M., Rupp, R., Liebetanz, D. (2014). P253: Myoelectric control by auricular muscles - an alternative human-machine interface. Clin. Neurophysiol., Supplement, S116. https://doi.org/10.1016/S1388-2457(14)50378-1

Tuga, M., Rupp, R., Liebetanz, D., Schmalfuß, L., Hübner, E., Doneit, W., Mikut, R., Reischl, M. (2013). Co-Adaptives Lernen: Untersuchungen einer Mensch-MaschineSchnittstelle mit anpassungsfähigem Systemverhalten. In Proc. 23. Workshop Computational Intelligence. 